\title{
LOCAL LUMINOUS INFRARED GALAXIES. I. SPATIALLY RESOLVED OBSERVATIONS WITH THE SPITZER INFRARED SPECTROGRAPH*
}

\author{
Miguel Pereira-Santaella ${ }^{1}$, Almudena Alonso-Herrero ${ }^{1,2,6}$, George H. Rieke ${ }^{3}$, Luis Colina ${ }^{1}$, Tanio Díaz-Santos ${ }^{1,7}$, \\ J.-D. T. Smith ${ }^{4}$, Pablo G. PÉrez-González ${ }^{2,5,6}$, And Charles W. Engelbracht ${ }^{3}$ \\ ${ }^{1}$ Instituto de Estructura de la Materia, CSIC, Serrano 121, E-28006, Madrid, Spain; pereira@ damir.iem.csic.es \\ ${ }^{2}$ Steward Observatory, University of Arizona, AZ 85721, USA \\ ${ }^{3}$ Steward Observatory, University of Arizona, 933 N. Cherry Avenue, Tucson, AZ 85721, USA \\ ${ }^{4}$ Ritter Astrophysical Research Center, University of Toledo, 2801 West Bancroft Street, Toledo, OH 43606, USA \\ ${ }^{5}$ Departamento de Astrofísica, Facultad de CC. Físicas, Universidad Complutense de Madrid, E-28040 Madrid, Spain \\ Received 2009 August 10; accepted 2010 March 30; published 2010 May 28
}

\begin{abstract}
We present results from the Spitzer Infrared Spectrograph spectral mapping observations of 15 local luminous infrared galaxies (LIRGs). In this paper, we investigate the spatial variations of the mid-IR emission which includes fine structure lines, molecular hydrogen lines, polycyclic aromatic features (PAHs), continuum emission, and the $9.7 \mu \mathrm{m}$ silicate feature. We also compare the nuclear and integrated spectra. We find that the star formation takes place in extended regions (several kpc) as probed by the PAH emission, as well as the [Ne II] $12.81 \mu \mathrm{m}$ and $[\mathrm{Ne} \mathrm{III}] 15.56 \mu \mathrm{m}$ emissions. The behavior of the integrated PAH emission and $9.7 \mu \mathrm{m}$ silicate feature is similar to that of local starburst galaxies. We also find that the minima of the $[\mathrm{Ne}$ III] $] 15.56 \mu \mathrm{m} /[\mathrm{Ne}$ II $] 12.81 \mu \mathrm{m}$ ratio tends to be located at the nuclei and its value is lower than that of H II regions in our LIRGs and nearby galaxies. It is likely that increased densities in the nuclei of LIRGs are responsible for the smaller nuclear [Ne III] $15.56 \mu \mathrm{m} /[\mathrm{Ne} \mathrm{II}] 12.81 \mu \mathrm{m}$ ratios. This includes the possibility that some of the most massive stars in the nuclei are still embedded in ultracompact $\mathrm{H}_{\text {II }}$ regions. In a large fraction of our sample, the $11.3 \mu \mathrm{m}$ PAH emission appears more extended than the dust $5.5 \mu \mathrm{m}$ continuum emission. We find a dependency of the $11.3 \mu \mathrm{m}$ $\mathrm{PAH} / 7.7 \mu \mathrm{m}$ PAH and [Ne II] $12.81 \mu \mathrm{m} / 11.3 \mu \mathrm{m}$ PAH ratios with the age of the stellar populations. Smaller and larger ratios, respectively, indicate recent star formation. The estimated warm $(300 \mathrm{~K}<T<1000 \mathrm{~K})$ molecular hydrogen masses are of the order of $10^{8} M_{\odot}$, which are similar to those found in ULIRGs, local starbursts, and Seyfert galaxies. Finally we find that the [Ne II] $12.81 \mu \mathrm{m}$ velocity fields for most of the LIRGs in our sample are compatible with a rotating disk at $\sim \mathrm{kpc}$ scales, and they are in a good agreement with $\mathrm{H} \alpha$ velocity fields.
\end{abstract}

Key words: galaxies: active - galaxies: nuclei - galaxies: starburst - galaxies: structure - infrared: galaxies

Online-only material: color figures, extended figures

\section{INTRODUCTION}

Infrared (IR) bright galaxies were first identified almost 40 years ago (Rieke \& Low 1972). The IRAS satellite detected a large number of these galaxies in the local universe (see Sanders \& Mirabel 1996 for a review). Galaxies were classified according to their IR $(8-1000 \mu \mathrm{m})$ luminosities into luminous infrared galaxies (LIRGs; $10^{11} L_{\odot}<L_{\mathrm{IR}}<10^{12} L_{\odot}$ ) and ultra luminous infrared galaxies (ULIRGs; $L_{\mathrm{IR}}>10^{12} L_{\odot}$ ). Since then, an increasing number of studies showed the important role of (U)LIRGs at cosmological distances. LIRGs and ULIRGs are not common in the local universe, accounting for just 5\% and $<1 \%$, respectively, of the total IR emission of galaxies. However, at $z \sim 1$, LIRGs dominate the IR background and the comoving star formation rate density, while ULIRGs are dominant at $z \sim 2$ (Le Floc'h et al. 2005; Pérez-González et al. 2005; Caputi et al. 2007).

Thanks to the high sensitivity of the Infrared Spectrograph (IRS; Houck et al. 2004) on board Spitzer, we can, for the first time, study systematically the mid-IR properties of (U)LIRGs, both locally and at high- $z$. The majority of IRS local studies

\footnotetext{
* This work is based on observations made with the Spitzer Space Telescope, which is operated by the Jet Propulsion Laboratory, California Institute of Technology under NASA contract 1407.

6 Associate Astronomer.

7 Current address: Department of Physics, University of Crete, GR-71003, Heraklion, Greece.
}

have, so far, focused on the properties of starburst galaxies (Smith et al. 2007b; Brandl et al. 2006) and ULIRGs (Armus et al. 2007; Farrah et al. 2007). The latter class of galaxies includes a large variety of physical and excitation conditions. For instance, most of these local $(z<0.3)$ ULIRGs are powered predominantly by compact starburst events, although a substantial active galactic nucleus (AGN) contribution is present in about half of them (Imanishi et al. 2007; Farrah et al. 2007; Nardini et al. 2008). In the future, the larger sample ( 200) of local LIRGs of the Spitzer legacy program GOALS will enable a statistical study of this class of galaxies (Armus et al. 2009).

Although ULIRGs at $z \sim 2$ extend to even higher luminosities than local ones, their mid-IR spectra are similar to those of local starbursts, rather than to local ULIRGs (Rigby et al. 2008; Farrah et al. 2008). A similar behavior is found for high- $z$ submillimeter galaxies (Pope et al. 2007; Menéndez-Delmestre et al. 2009). This behavior may indicate that star formation in IR-bright galaxies at $z \sim 2$ takes place over larger scales (a few kpc) than in local ULIRGs, where most of the IR emission arises from very compact regions (sub-kiloparsec scales, see, e.g., Soifer et al. 2000; Farrah et al. 2007).

As a result, local LIRGs may provide important insights to the behavior of high-redshift ULIRGs. Their star formation is often distributed over a substantial fraction of the galaxy (e.g., Alonso-Herrero et al. 2006), and in any case is usually not embedded at the high optical depths characteristic of the nuclear star-forming regions in local ULIRGs. To make good 
Table 1

Log of Spitzer/IRS Spectral Mapping Observations

\begin{tabular}{|c|c|c|c|c|c|c|c|c|c|}
\hline Target & IRAS Name & $\begin{array}{c}\text { R.A. }^{\mathrm{a}} \\
(\mathrm{J} 2000.0)\end{array}$ & $\begin{array}{c}\text { Decl. }^{\mathrm{a}} \\
(\mathrm{J} 2000.0)\end{array}$ & $\begin{array}{c}\mathrm{v}_{\text {hel }}{ }^{\mathrm{b}} \\
\left(\mathrm{km} \mathrm{s}^{-1}\right)\end{array}$ & $\begin{array}{l}\text { Dist. }^{\mathrm{c}} \\
(\mathrm{Mpc})\end{array}$ & $\begin{array}{c}\text { Scale } \\
\left(\mathrm{pc} \operatorname{arcsec}^{-1}\right)\end{array}$ & $L_{\mathrm{IR}}^{\mathrm{d}}$ & $\begin{array}{l}\text { Spect. } \\
\text { Class }\end{array}$ & $\mathrm{AOR}^{\mathrm{f}}$ \\
\hline NGC 2369 & IRAS F07160-6215 & 071637.75 & -622037.5 & 3196 & 46.0 & 220 & 11.1 & $\ldots$ & 17659392 \\
\hline NGC 3110 & IRAS F10015-0614 & 100402.12 & -062829.1 & 5014 & 72.5 & 350 & 11.2 & H II & 17659648 \\
\hline NGC 3256 & IRAS F10257-4339 & 102751.28 & -435413.5 & 2790 & 40.1 & 190 & 11.6 & $\mathrm{H}_{\text {II }}$ & 17659904 \\
\hline NGC $3690^{g}$ & IRAS F11257+5850 & 112830.80 & +583344.0 & 3121 & 44.9 & 220 & 11.8 & Sy2 & 17660160 \\
\hline IC $694^{g}$ & & 112833.80 & +583346.5 & & & & & $\mathrm{H}_{\text {II }}$ & 17660416 \\
\hline ESO 320-G030 & IRAS F11506-3851 & 115311.72 & -390748.7 & 3038 & 43.7 & 210 & 11.2 & H II & 17660672 \\
\hline NGC 5135 & IRAS F13229-2934 & 132544.06 & -295001.2 & 4074 & 58.8 & 280 & 11.2 & Sy2 & 17660928 \\
\hline IC $4518 \mathrm{E}$ & IRAS F14544-4255 & 145744.80 & -430753.0 & 4584 & 64.5 & 320 & 11.2 & $\ldots$ & 21927936 \\
\hline Zw049.057 & IRAS F15107+0724 & 151313.12 & +071331.7 & 3858 & 55.6 & 270 & 11.2 & H II & 17661184 \\
\hline IRAS $17138-1017$ & IRAS F17138-1017 & 171635.80 & -102039.4 & 5161 & 74.7 & 360 & 11.4 & H II & 17661440 \\
\hline IC 4687 & IRAS F18093-5744 & 181339.83 & -574331.2 & 5105 & 73.8 & 360 & 11.5 & H II & 17665024 \\
\hline NGC 6701 & IRAS F18425+6036 & 184312.46 & +603912.0 & 3896 & 56.2 & 270 & 11.0 & Composite & 21928448 \\
\hline NGC 7130 & IRAS F21453-3511 & 214819.40 & -345704.9 & 4837 & 69.9 & 340 & 11.4 & LINER/Sy2 & 17661696 \\
\hline IC 5179 & IRAS F22132-3705 & 221609.09 & -365037.5 & 3363 & 48.4 & 230 & 11.2 & H II & 17661952 \\
\hline NGC 7591 & IRAS F23157+0618 & 231816.28 & +063508.9 & 4907 & 70.9 & 340 & 11.0 & Composite & 21928960 \\
\hline NGC 7771 & IRAS F23488+1949 & 235124.88 & +200642.6 & 4302 & 62.1 & 300 & 11.4 & H II & 21929472 \\
\hline
\end{tabular}

Notes.

a Coordinates of the nucleus of the galaxy.

${ }^{b}$ Heliocentric velocity from the IRS nuclear spectrum of each galaxy.

${ }^{c}$ Distance calculated using the measured heliocentric velocity and our cosmology.

${ }^{\mathrm{d}}$ Logarithm of the $L(8-1000 \mu \mathrm{m})$, as defined in Sanders \& Mirabel (1996), in solar luminosities $\left(3.826 \times 10^{26} \mathrm{~W}\right)$. It is calculated using the IRAS fluxes from Sanders et al. (2003) and from Surace et al. (2004), the measured redshift, and our cosmology.

e Classification of the nuclear activity from optical spectroscopy. Composite indicates intermediate LINER/H II classification. The references for the nuclear classification are given in Alonso-Herrero et al. (2006), except for NGC 3690 (García-Marín et al. 2006), NGC 6701 and NGC 7591 (Alonso-Herrero et al. 2009a).

f Astronomical Observation Request key.

g NGC 3690 and IC 694 are the two components, western and eastern respectively, of the interacting system Arp 299.

use of this analogy requires observations that map the local LIRGs to get an understanding of their global properties as well as to provide a better comparison with the observations of high- $z$ galaxies, where the entire galaxy is encompassed in the IRS slit. The spectral mapping mode of Spitzer/IRS is well suited for this purpose, since it can provide both the nuclear and integrated spectra of local galaxies, enabling study of the spatial distribution of the spectral features that make up the integrated spectrum.

This is the first in a series of papers studying the mid-IR properties of local LIRGs. We present Spitzer/IRS spectral mapping observations of a representative sample of 15 local LIRGs. The first results and the goals of this program were presented by Alonso-Herrero et al. (2010). In the present paper we focus on the analysis of the spatially resolved measurements. The paper is organized as follows. In Section 2, we present the sample and the observational details. Section 3 describes the analysis of the data. We examine the silicate feature in Section 4. Sections 5-7 discuss the spatially resolved properties of the atomic fine structure lines, PAHs, and molecular hydrogen emission, respectively. We explore the velocity fields in Section 8. Finally, in Section 9 we summarize the main results. Throughout this paper we assume a flat cosmology with $H_{0}=70 \mathrm{~km} \mathrm{~s}^{-1} \mathrm{Mpc}^{-1}, \Omega_{M}=0.3$, and $\Omega_{\Lambda}=0.7$.

\section{OBSERVATIONS AND DATA REDUCTION}

\subsection{The Sample}

The galaxies studied here were selected from the sample of 30 local LIRGs described in Alonso-Herrero et al. (2006), which was drawn from the IRAS Revised Bright Galaxy Sample (RBGS; Sanders et al. 2003). The sample of Alonso-Herrero et al. (2006) is a volume limited sample, and the velocity range was selected so that the $\mathrm{Pa} \alpha$ emission line could be observed with the NICMOS F190N filter on board the Hubble Space Telescope (HST). This restricted the sample to local LIRGs (40 Mpc $\lesssim d \lesssim 75 \mathrm{Mpc}$ ). From this sample we selected 15 galaxies (Table 1) with extended emission in the NICMOS Pa $\alpha$ images to be mapped with the IRS spectrograph. This selection criterion does not introduce any bias in the luminosity, distance, and nuclear activity distributions of the mapped galaxies as compared to the parent sample of Alonso-Herrero et al. (2006). The remaining galaxies in the sample have been observed using the IRS staring mode as part of different programs. The latter will be analyzed with the mapping data in a forthcoming paper (M. Pereira-Santaella et al. 2010, in preparation). The interacting galaxy Arp 299 was studied in detail by Alonso-Herrero et al. (2009b) and will be included in the appropriate comparisons and figures in this paper.

Most of the nuclei of the LIRGs in our sample are classified as $\mathrm{H}$ II region-like from optical spectroscopy, three galaxies have a Seyfert classification, and two are classified as composite (intermediate between H II and LINER, see Alonso-Herrero et al. 2009a). The infrared luminosity of the sample ranges from $10^{11.0}$ to $10^{11.8} L_{\odot}$. The main properties of the galaxies are summarized in Table 1.

\subsection{Spectral Mapping}

We obtained Spitzer IRS spectral mapping observations through two guaranteed time observation (GTO) programs P30577 and P40479 (PI: G. H. Rieke). We used all four IRS modules: high-resolution $(R \sim 600)$ modules with short-high (SH; 9.9-19.6 $\mu \mathrm{m})$ and long-high (LH; 18.7-37.2 $\mu \mathrm{m})$, and low 
Table 2

Spitzer/IRS Mapping Observation Details

\begin{tabular}{lccccc}
\hline \hline IRS Module & $\begin{array}{c}\text { Pixel Size } \\
(\operatorname{arcsec})\end{array}$ & $\begin{array}{c}\text { Ramp Duration } \\
(\mathrm{s})\end{array}$ & Number of Cycles & $\begin{array}{c}\text { Step Size Perpendicular } \\
(\operatorname{arcsec})\end{array}$ & $\begin{array}{c}\text { Step Size Parallel } \\
(\operatorname{arcsec})\end{array}$ \\
\hline SL1, SL2 & 1.85 & 14 & 2 & 1.8 & $\ldots$ \\
LL1, LL2 & 4.46 & 14 & 2 & 5.25 & $\ldots$ \\
SH & 2.26 & 30 & 2 & 2.35 & 5.65 \\
LH & 4.46 & 60 & 4 & 5.55 & $\ldots$ \\
\hline
\end{tabular}

resolution $(R \sim 60-120)$ with short-low (SL1; 7.4-14.5 $\mu \mathrm{m}$, SL2; 5.2-7.7 $\mu \mathrm{m}$ ) and long-low (LL1; 19.5-38.0 $\mu \mathrm{m}$, LL2; $14.0-21.3 \mu \mathrm{m})$. Maps were made by moving the telescope pointing position perpendicular to the long axis of the slit with a step size half of the slit width. The SL, LL, and LH slits are long enough to cover the full region of interest with just one slit length, but due to the smaller size of SH slit, parallel steps were also needed. The parallel step size was half of the slit height. This strategy produced maps with a redundancy per pixel of 2 for SL, LL, and LH, and 4 for SH. Table 2 details the integration time, pixel size and step size for each IRS module. The maps cover at least the central $20^{\prime \prime} \times 20^{\prime \prime}$ to $30^{\prime \prime} \times 30^{\prime \prime}$ of the galaxies.

Dedicated background observations are not needed for the low-resolution modules (SL and LL) because the separation on the sky between the two orders of each module is larger than the galaxy size. That is, when order 1 is on the galaxy, order 2 is observing the background and vice versa. The $\mathrm{SH}$ and LH slits are too small to allow background subtraction. We obtained dedicated background observations for LH by observing a region 2 arcmin away from the galaxy. SH sky observations are available only for the galaxies observed under the program P40479. For the galaxies without dedicated SH background observations, we estimated the sky background emission by combining the background spectra from the lowresolution modules. Generally the sky contribution is not very important for the galaxies in our sample, except for NGC 3110 and $\mathrm{Zw}$ 049.057. The sky background is bright at the position of these galaxies and its subtraction is essential to match the fluxes from the different slits. Moreover, subtracting background observations is always useful because it removes many of the rogue pixels and improves the final quality of the spectra. This is especially important for the LH module since it contains a large number of such pixels.

We used Basic Calibrated Data (BCD), processed by Spitzer pipeline version S15.3 (low-resolution modules: SL and LL) and version S17.2 (high-resolution modules: SH and $\mathrm{LH}$ ) to build data cubes with CUBISM (Smith et al. 2007a). CUBISM is a tool that combines slit observations to create spectral cubes. It also performs background subtraction, flux calibration, and estimates the statistical uncertainty at each spectral wavelength.

\section{ANALYSIS}

\subsection{SL Spectral Maps}

Using the SL cubes generated by CUBISM we constructed maps of the $5.5 \mu \mathrm{m}$ continuum, and the most prominent aromatic features (hereafter PAH) in this module $(6.2 \mu \mathrm{m} \mathrm{PAH}, 7.7 \mu \mathrm{m}$ PAH and $11.3 \mu \mathrm{m}$ PAH). To build them we used our own IDL procedures. First we extracted one-dimensional spectra from the cubes by averaging the spectra over a $2 \times 2$ pixel aperture and then by moving with a 1 pixel step until the whole field of view was covered. The $2 \times 2$ pixel extraction box allows us to increase the signal-to-noise ratio $(\mathrm{S} / \mathrm{N})$ of the one-dimensional spectra, with only a slight loss of angular resolution (see below and Appendix A). Once we obtained the one-dimensional spectra, for each pixel we did the following. To create the $5.5 \mu \mathrm{m}$ continuum map, we integrated the flux between the rest-frame $5.3 \mu \mathrm{m}$ and $5.7 \mu \mathrm{m}$ wavelengths (in this section all wavelengths refer to rest-frame wavelengths). For the PAH maps, first we fitted the local continuum using a linear fit. The continuum pivots were set by averaging the spectrum over a $\sim 0.2 \mu \mathrm{m}$ interval centered at $5.75 \mu \mathrm{m}$ and $6.7 \mu \mathrm{m}$ for the $6.2 \mu \mathrm{m} \mathrm{PAH}$, at $7.2 \mu \mathrm{m}$ and $8.2 \mu \mathrm{m}$ for the $7.7 \mu \mathrm{m} \mathrm{PAH}$, and at $10.6 \mu \mathrm{m}$ and $11.8 \mu \mathrm{m}$ for the $11.3 \mu \mathrm{m}$ PAH. After subtracting the continuum, we integrated the flux in the wavelength ranges between 5.9 and $6.5 \mu \mathrm{m} 7.3$ and $7.9 \mu \mathrm{m}$ and 10.8 and $11.6 \mu \mathrm{m}$ for the $6.2,7.7$ and $11.3 \mu \mathrm{m}$ PAH features, respectively. We derived the flux uncertainty of these maps with the statistical uncertainties provided by CUBISM. To avoid noisy pixels, we required the feature peak to be at least above $1 \sigma$ to plot that pixel in the map, where $\sigma$ is the continuum noise. The SL spectral maps of our sample are shown in Figure 1.

The angular resolution (FWHM) of the SL spectral maps is $\sim 4$ ". 5 . This is the resolution when we use $2 \times 2$ boxes to construct the maps, as opposed to that measured from the standard star observations (see Appendix A). For the distances of our galaxies this angular resolution corresponds to $\sim 1 \mathrm{kpc}$ (the exact scale for each galaxy is given in Table 1). In Appendix A, we give full details on how the angular resolution of the spectral maps was derived.

We constructed maps of different PAH ratios by dividing the PAH maps obtained as described above. To avoid noisy pixels in the ratio maps we imposed further restrictions, and only those pixels with $\mathrm{S} / \mathrm{N}$ greater than 4 are plotted in the PAH ratio maps. Figures 2 and 3 show the $11.3 \mu \mathrm{m} \mathrm{PAH} / 6.2 \mu \mathrm{m}$ PAH and $11.3 \mu \mathrm{m} \mathrm{PAH} / 7.7 \mu \mathrm{m}$ PAH ratios, respectively.

\subsection{SH Spectral Maps}

We used the $\mathrm{SH}$ cubes to measure the brightest fine structure emission lines: [S IV] $10.51 \mu \mathrm{m},[\mathrm{Ne}$ II] $12.81 \mu \mathrm{m}$, [Ne III] $15.56 \mu \mathrm{m},[\mathrm{S}$ III] $18.71 \mu \mathrm{m}$ and the molecular hydrogen lines, $\mathrm{H}_{2} \mathrm{~S}(2)$ at $12.3 \mu \mathrm{m}$ and $\mathrm{H}_{2} \mathrm{~S}(1)$ at $17.0 \mu \mathrm{m}$. First we extracted one-dimensional spectra using the same method as for the low-resolution cubes. Then we used the IDL package MPFIT (Markwardt 2009) to fit simultaneously a first-order polynomial to the continuum and a Gaussian to the line. The fit was over a wavelength range of $\sim 0.4 \mu \mathrm{m}$ centered at the line wavelength. The spectral resolution, $R \sim 600$, is sufficiently high to allow us to separate the emission lines from the broad PAH bands. However, to improve the continuum determination, we used extra Gaussian profiles to fit the PAH features close to the emission lines (e.g., the $12.7 \mu \mathrm{m}$ PAH feature next to the [Ne II]). To avoid noisy pixels in the maps, we required (1) an $\mathrm{S} / \mathrm{N}>2$ in the continuum range; and (2) the line peak to be at least $1 \sigma$ above the continuum noise for the pixel to be shown in the corresponding map. 

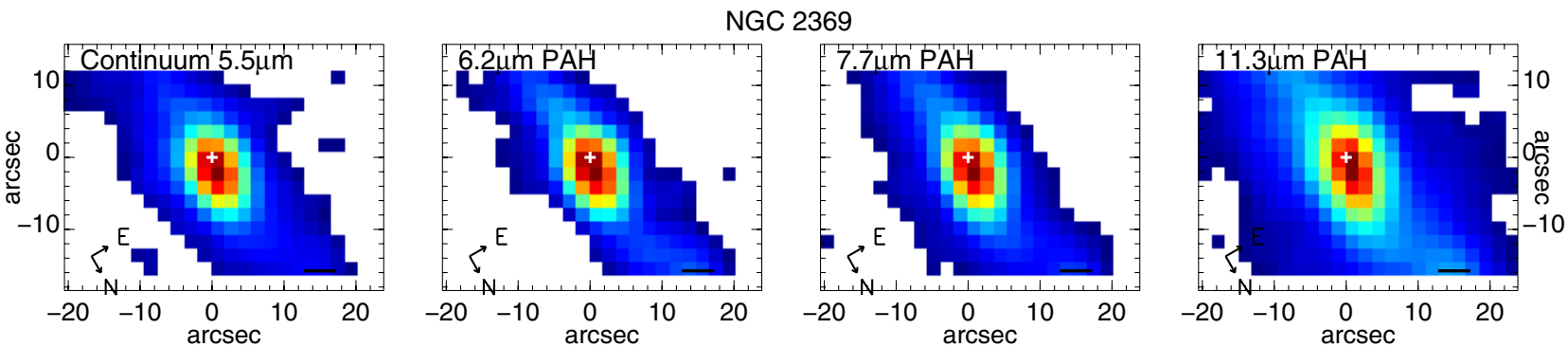

Figure 1. Spitzer/IRS SL spectral maps of the $5.5 \mu \mathrm{m}$ continuum, the $6.2 \mu \mathrm{m}$ PAH, the $7.7 \mu \mathrm{m} \mathrm{PAH}$, and the $11.3 \mu \mathrm{m}$ PAH. The white cross marks the coordinates of the nucleus as listed in Table 1. The image orientation is indicated on the maps for each galaxy. The scale represents $1 \mathrm{kpc}$. The maps are shown in a square root scale. (A color version and an extended version of this figure are available in the online journal.)
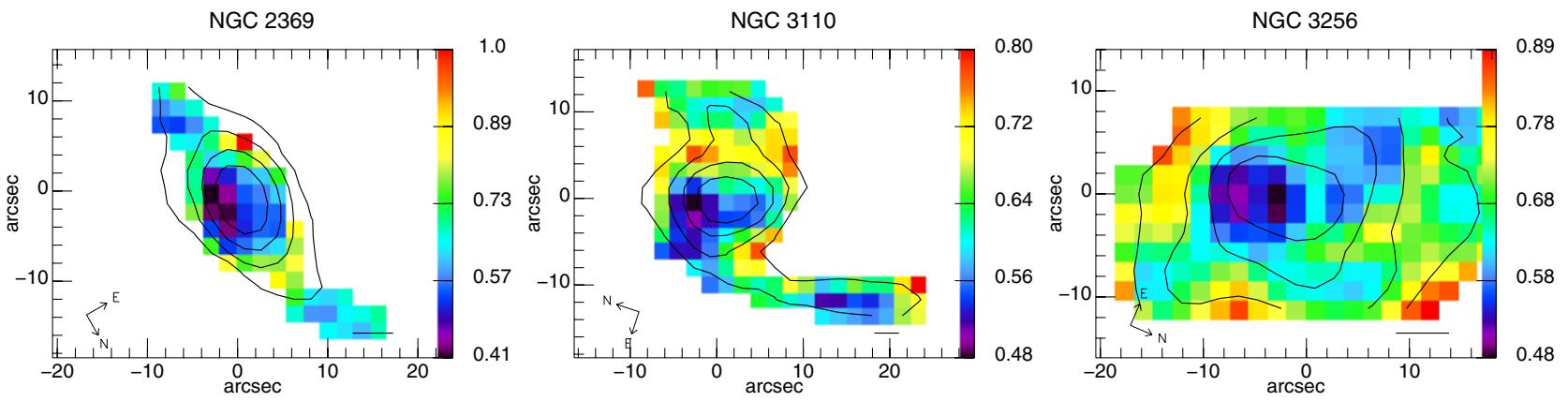

Figure 2. Spitzer/IRS SL spectral maps of the $11.3 \mu \mathrm{m}$ PAH/6.2 $\mu \mathrm{m}$ PAH ratio. The $5.5 \mu \mathrm{m}$ continuum contours, in a logarithm scale, are displayed to guide the eye. The image orientation is indicated on the maps for each galaxy. The scale represents $1 \mathrm{kpc}$. The ratio maps are shown in a linear scale.

(A color version and an extended version of this figure are available in the online journal.)
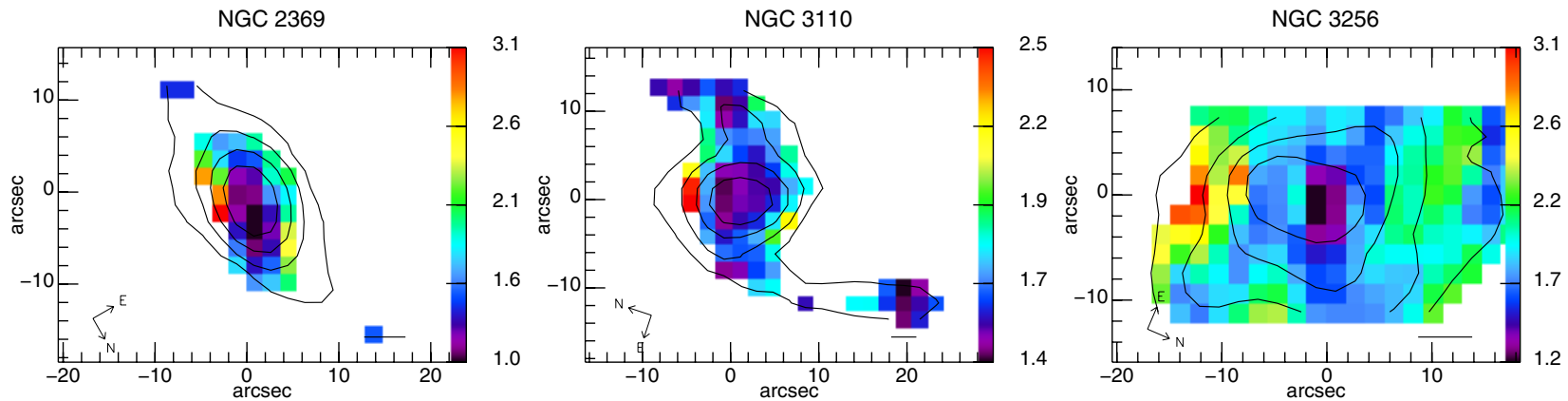

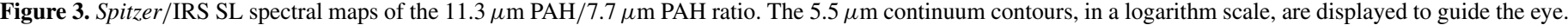
The image orientation is indicated on the maps for each galaxy. The scale represents $1 \mathrm{kpc}$. The ratio maps are shown in a linear scale.

(A color version and an extended version of this figure are available in the online journal.)

We also constructed an $11.3 \mu \mathrm{m}$ PAH feature map by the same procedure as for SL cubes, but with the high-resolution spectra. A $15 \mu \mathrm{m}$ continuum was obtained by integrating the flux between $14.9 \mu \mathrm{m}$ and $15.1 \mu \mathrm{m}$. We required a signal above $1 \sigma$ to plot a pixel in these two maps.

The SH spectral maps are shown in Figure 4 together with the HST/NICMOS continuum-subtracted Pa $\alpha$ maps of AlonsoHerrero et al. (2006).

We constructed [S III] $18.71 \mu \mathrm{m} /[\mathrm{Ne}$ II $] 12.81 \mu \mathrm{m}, \quad[\mathrm{Ne}$ III] $15.56 \mu \mathrm{m} /[\mathrm{Ne}$ II] $12.81 \mu \mathrm{m}$, and [Ne II] $12.81 \mu \mathrm{m} / 11.3 \mu \mathrm{m} \mathrm{PAH}$ ratio maps by dividing the flux maps. The variation of the pointspread function (PSF) size with wavelength within each module is negligible (see Appendix A), and therefore we divided the flux maps directly without attempting to correct for this small effect (we only built ratio maps of lines observed with the SH module). We imposed stricter conditions to plot a pixel in a ratio map than those used for the line maps; only pixels with a flux measurement $>3 \sigma$, for both features, were included in the ratio maps. The maps of the SH line ratios are shown in
Figure 5. The angular resolution of the SH maps is $\sim 6$ arcsec. This corresponds to physical scales between $\sim 1 \mathrm{kpc}$, for the nearest galaxies, and $\sim 2 \mathrm{kpc}$ for the most distant ones.

\subsection{Nuclear, Integrated, and Extranuclear One-dimensional Spectra}

We extracted one-dimensional spectra of the nuclei as well as of the integrated galaxy. To extract the nuclear spectra we used a $5^{\prime \prime} .5 \times 5^{\prime \prime} .5$ square aperture centered at the nuclear coordinates (Table 1). This aperture corresponds to physical scales between 1 and $2 \mathrm{kpc}$, depending on the galaxy. We set the orientation of the aperture aligned with the R.A. and decl. axis. We used the same aperture for both low- and high-resolution data cubes. To obtain the integrated spectrum we summed the flux of the cube. We excluded those pixels with the $11.3 \mu \mathrm{m}$ PAH flux $(15 \mu \mathrm{m}$ continuum) below $1 \sigma$ to avoid adding too much noise to the lowresolution (high-resolution) integrated spectra. This threshold is 

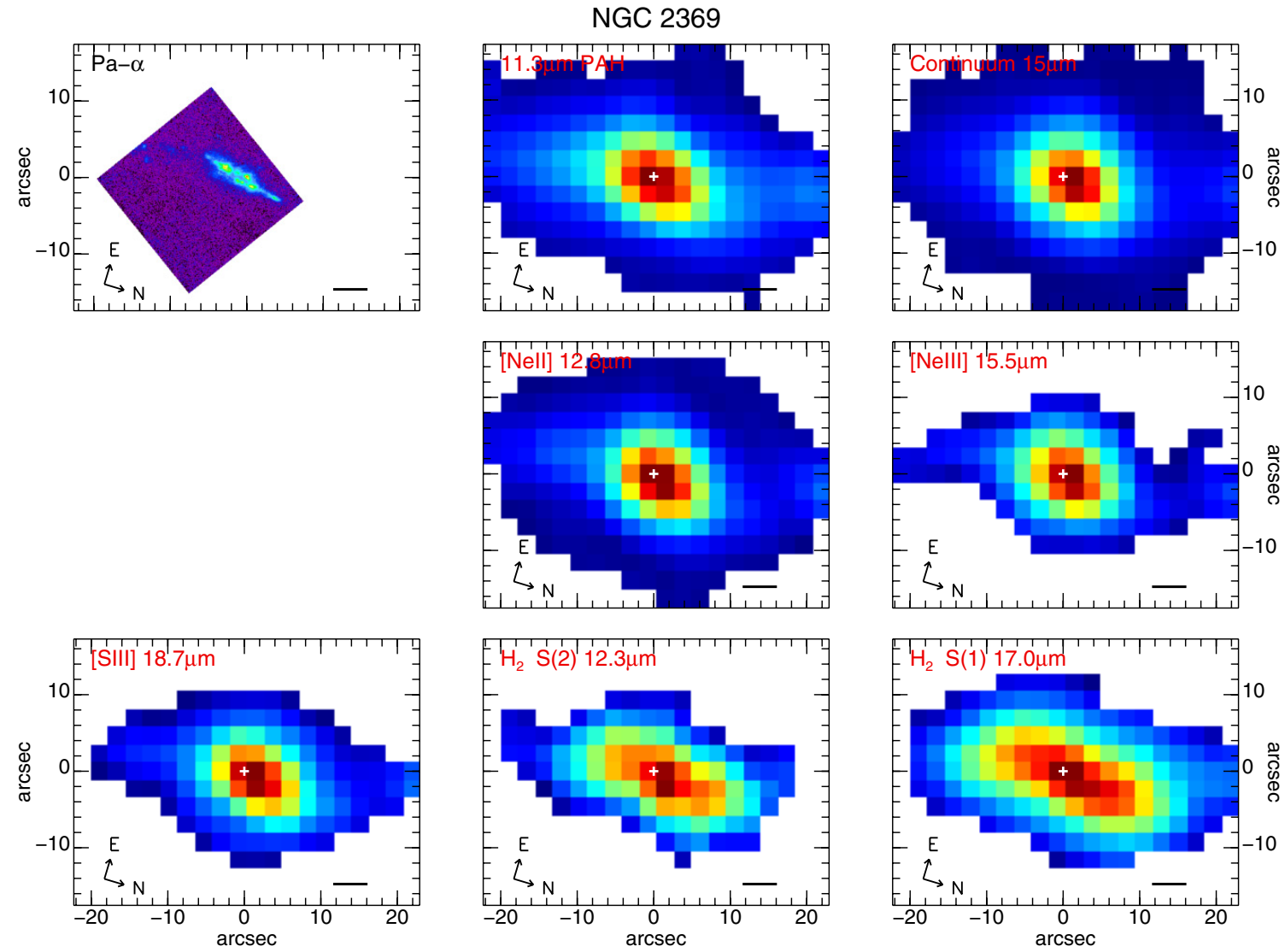

Figure 4. Spitzer/IRS SH spectral maps of the $11.3 \mu \mathrm{m}$ PAH feature, the $15 \mu \mathrm{m}$ continuum, the fine structure line emission of [S IV] $10.51 \mu \mathrm{m}$, [Ne II] $12.81 \mu \mathrm{m}$, $\left[\mathrm{Ne}\right.$ III] $15.56 \mu \mathrm{m}$, and $[\mathrm{S} \mathrm{III}] 18.71 \mu \mathrm{m}$, and the molecular hydrogen lines $\mathrm{H}_{2} \mathrm{~S}(2)$ at $12.3 \mu \mathrm{m}$ and $\mathrm{H}_{2} \mathrm{~S}(1)$ at $17.0 \mu \mathrm{m}$. The white cross marks the coordinates of the nucleus as listed in Table 1. The image orientation is indicated on the maps for each galaxy. The scale represents $1 \mathrm{kpc}$. The maps are shown in a square root scale. (A color version and an extended version of this figure are available in the online journal.)
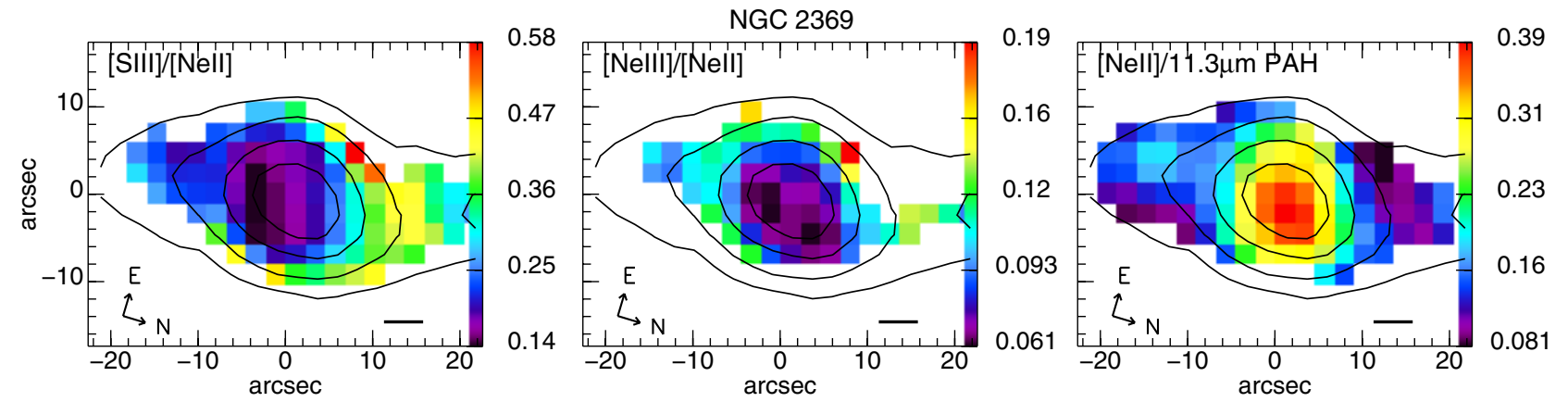

Figure 5. Spitzer/IRS SH spectral maps of the [S III] $18.71 \mu \mathrm{m} /[\mathrm{Ne}$ II] $12.81 \mu \mathrm{m}$ ratio, the [Ne III] $15.56 \mu \mathrm{m} /[\mathrm{Ne}$ II] $12.81 \mu \mathrm{m}$ ratio, and the [Ne II] $12.81 \mu \mathrm{m} / 11.3 \mu \mathrm{m}$ PAH. The $15.0 \mu \mathrm{m}$ continuum contours are displayed to guide the eye. The image orientation is indicated on the maps for each galaxy. The scale represents $1 \mathrm{kpc}$. The ratio maps are shown in a linear scale.

(A color version and an extended version of this figure are available in the online journal.)

the same as that required to plot a pixel in the $11.3 \mu \mathrm{m}$ PAH and $15 \mu \mathrm{m}$ continuum maps of Figures 1 and 4, respectively. The physical scale of the integration area varies from galaxy to galaxy. The approximated physical sizes are listed in Tables 3 and 4 for the low- and high-resolution maps, respectively. They are of the order of $10 \times 8 \mathrm{kpc}$. Although the extraction regions are slightly different for each module, the flux differences are only $\sim 10 \%-20 \%$.

We plot the integrated spectra in Figure 6. The nuclear spectra will be presented in M. Pereira-Santaella et al. (2010, in preparation), together with the nuclear spectra of the rest of the Alonso-Herrero et al. (2006) sample.
In Figure 7 (right), we show a comparison of the nuclear and extranuclear low-resolution spectra normalized at $14 \mu \mathrm{m}$ for four of the galaxies. We selected these galaxies because in their spectral maps (left panel of Figure 7) it is possible to identify extranuclear $\mathrm{H}$ in regions and extract their spectra. All of the spectra, nuclear and extranuclear, show bright PAH features and fine structure lines. The variations of the spectral features from galaxy to galaxy and within a galaxy are relatively small, at least when compared to those observed in ULIRGs (Armus et al. 2007) or those observed in star-forming galaxies with different metallicities (Engelbracht et al. 2008). That is, the comparison of the spectra shows that these LIRGs have a more 
Table 3

Low-resolution (SL) Spectra

\begin{tabular}{|c|c|c|c|c|c|c|c|c|c|c|c|c|}
\hline \multirow[t]{3}{*}{ Name } & \multicolumn{6}{|c|}{ Nuclear } & \multicolumn{6}{|c|}{ Integrated } \\
\hline & \multirow[b]{2}{*}{ Size $^{\mathrm{b}}$} & \multirow[b]{2}{*}{$S_{\mathrm{Si}}$} & \multicolumn{2}{|c|}{ PAH EW ${ }^{\mathrm{a}}$} & \multirow[b]{2}{*}{$\begin{array}{l}7.7 \mu \mathrm{m} \text { PAH } \\
6.2 \mu \mathrm{m} \mathrm{PAH} \\
\end{array}$} & \multirow[b]{2}{*}{$\frac{11.3 \mu \mathrm{m} \mathrm{PAH}}{6.2 \mu \mathrm{m} \mathrm{PAH}}$} & \multirow[b]{2}{*}{$\mathrm{Size}^{\mathrm{c}}$} & \multirow[b]{2}{*}{$S_{\mathrm{Si}}$} & \multicolumn{2}{|c|}{ PAH EW } & \multirow[b]{2}{*}{$\begin{array}{l}7.7 \mu \mathrm{m} \text { PAH } \\
6.2 \mu \mathrm{m} \mathrm{PAH} \\
\end{array}$} & \multirow[b]{2}{*}{$\frac{11.3 \mu \mathrm{m} \mathrm{PAH}}{6.2 \mu \mathrm{m} \mathrm{PAH}}$} \\
\hline & & & $6.2 \mu \mathrm{m}$ & $11.3 \mu \mathrm{m}$ & & & & & $6.2 \mu \mathrm{m}$ & $11.3 \mu \mathrm{m}$ & & \\
\hline NGC 2369 & 1.2 & -0.92 & $0.43 \pm 0.04$ & $0.33 \pm 0.02$ & $1.8 \pm 0.0$ & $0.38 \pm 0.01$ & $8.8 \times 6.2$ & -0.84 & $0.43 \pm 0.08$ & $0.40 \pm 0.03$ & $1.8 \pm 0.0$ & $0.55 \pm 0.01$ \\
\hline NGC 3110 & 2.0 & -0.48 & $0.58 \pm 0.11$ & $0.48 \pm 0.05$ & $1.7 \pm 0.0$ & $0.46 \pm 0.01$ & $15.4 \times 9.8$ & -0.40 & $0.73 \pm 1.02$ & $0.53 \pm 0.05$ & $1.6 \pm 0.3$ & $0.49 \pm 0.06$ \\
\hline NGC 3256 & 1.1 & -0.48 & $0.53 \pm 0.02$ & $0.33 \pm 0.01$ & $1.7 \pm 0.0$ & $0.46 \pm 0.01$ & $7.6 \times 3.8$ & -0.52 & $0.59 \pm 0.02$ & $0.43 \pm 0.01$ & $1.7 \pm 0.0$ & $0.53 \pm 0.01$ \\
\hline NGC $3690^{\mathrm{d}}$ & 1.2 & -0.80 & $0.11 \pm 0.01$ & $0.045 \pm 0.001$ & $1.4 \pm 0.1$ & $0.21 \pm 0.01$ & $8.3 \times 5.7$ & -0.96 & $0.29 \pm 0.01$ & $0.21 \pm 0.01$ & $1.7 \pm 0.1$ & $0.42 \pm 0.03$ \\
\hline IC $694^{\mathrm{d}}$ & 1.2 & -1.9 & $0.34 \pm 0.01$ & $0.23 \pm 0.01$ & $2.1 \pm 0.1$ & $0.28 \pm 0.02$ & $8.3 \times 5.7$ & -0.96 & $0.29 \pm 0.01$ & $0.21 \pm 0.01$ & $1.7 \pm 0.1$ & $0.42 \pm 0.03$ \\
\hline ESO 320-G030 & 1.2 & -0.73 & $0.49 \pm 0.06$ & $0.50 \pm 0.03$ & $1.8 \pm 0.0$ & $0.55 \pm 0.01$ & $5.9 \times 4.6$ & -0.56 & $0.53 \pm 0.10$ & $0.53 \pm 0.04$ & $1.8 \pm 0.0$ & $0.63 \pm 0.01$ \\
\hline NGC 5135 & 1.6 & -0.47 & $0.30 \pm 0.02$ & $0.31 \pm 0.02$ & $1.7 \pm 0.0$ & $0.58 \pm 0.01$ & $10 \times 5.9$ & -0.46 & $0.33 \pm 0.05$ & $0.35 \pm 0.03$ & $1.7 \pm 0.0$ & $0.73 \pm 0.02$ \\
\hline IC $4518 \mathrm{E}$ & 1.8 & -0.82 & $0.39 \pm 0.28$ & $0.44 \pm 0.17$ & $1.8 \pm 0.2$ & $0.47 \pm 0.05$ & $6.4 \times 6.4$ & -0.65 & $0.62 \pm 0.39$ & $0.53 \pm 0.16$ & $1.5 \pm 0.1$ & $0.48 \pm 0.03$ \\
\hline Zw 049.057 & 1.5 & -1.2 & $0.39 \pm 0.15$ & $0.48 \pm 0.16$ & $2.1 \pm 0.1$ & $0.49 \pm 0.03$ & $4.9 \times 3.8$ & -1.2 & $0.42 \pm 0.30$ & $0.47 \pm 0.19$ & $2.1 \pm 0.2$ & $0.57 \pm 0.06$ \\
\hline IRAS 17138-1017 & 2.0 & -0.79 & $0.56 \pm 0.06$ & $0.40 \pm 0.03$ & $1.7 \pm 0.0$ & $0.38 \pm 0.01$ & $10 \times 4.3$ & -0.77 & $0.58 \pm 0.10$ & $0.43 \pm 0.04$ & $1.7 \pm 0.0$ & $0.48 \pm 0.01$ \\
\hline IC 4687 & 2.0 & -0.40 & $0.66 \pm 0.08$ & $0.55 \pm 0.03$ & $1.6 \pm 0.0$ & $0.49 \pm 0.01$ & $13 \times 5.8$ & -0.38 & $0.64 \pm 0.11$ & $0.54 \pm 0.04$ & $1.7 \pm 0.0$ & $0.57 \pm 0.01$ \\
\hline NGC 6701 & 1.5 & -0.54 & $0.46 \pm 0.07$ & $0.45 \pm 0.05$ & $1.7 \pm 0.0$ & $0.58 \pm 0.01$ & $13 \times 6.5$ & -0.40 & $0.53 \pm 0.12$ & $0.52 \pm 0.05$ & $1.6 \pm 0.0$ & $0.70 \pm 0.02$ \\
\hline NGC 7130 & 1.9 & -0.44 & $0.25 \pm 0.04$ & $0.23 \pm 0.04$ & $1.8 \pm 0.1$ & $0.66 \pm 0.02$ & $12 \times 8.2$ & -0.38 & $0.47 \pm 0.10$ & $0.37 \pm 0.05$ & $1.5 \pm 0.0$ & $0.65 \pm 0.02$ \\
\hline IC 5179 & 1.3 & -0.50 & $0.57 \pm 0.08$ & $0.46 \pm 0.03$ & $1.7 \pm 0.0$ & $0.48 \pm 0.01$ & $10 \times 5.5$ & -0.32 & $0.62 \pm 0.08$ & $0.51 \pm 0.03$ & $1.6 \pm 0.0$ & $0.54 \pm 0.01$ \\
\hline NGC 7591 & 1.9 & -0.85 & $0.39 \pm 0.12$ & $0.35 \pm 0.06$ & $1.9 \pm 0.1$ & $0.52 \pm 0.02$ & $16 \times 8.8$ & -0.47 & $0.63 \pm 0.31$ & $0.43 \pm 0.08$ & $1.4 \pm 0.1$ & $0.53 \pm 0.03$ \\
\hline NGC 7771 & 1.7 & -0.66 & $0.46 \pm 0.06$ & $0.38 \pm 0.04$ & $1.7 \pm 0.0$ & $0.43 \pm 0.01$ & $11 \times 7.8$ & -0.70 & $0.49 \pm 0.07$ & $0.41 \pm 0.03$ & $1.7 \pm 0.0$ & $0.51 \pm 0.01$ \\
\hline
\end{tabular}

Notes.

${ }^{\text {a }}$ Equivalent width units are $\mu \mathrm{m}$.

b Projected square size of the aperture in kpc. The aperture size is 5 "'5 $55^{\prime \prime} 5$ for all the galaxies.

${ }^{c}$ Approximated projected square size of the aperture in kpc. See Section 3.3 for details.

${ }^{\mathrm{d}}$ NGC 3690 and IC 694 are part of the interacting system Arp 299. 
Table 4

High-resolution (SH) Spectra: Integrated Spectra

\begin{tabular}{|c|c|c|c|c|c|c|c|c|}
\hline Name & Size $^{\mathrm{a}}$ & $\begin{array}{c}{\left[\mathrm{S}_{\mathrm{IV}}\right]} \\
10.51 \mu \mathrm{m}\end{array}$ & $\begin{array}{c}{\left[\mathrm{Ne} \mathrm{II}^{\mathrm{II}}\right]} \\
12.81 \mu \mathrm{m}\end{array}$ & $\begin{array}{c}{[\mathrm{Ne} \text { III] }} \\
15.55 \mu \mathrm{m}\end{array}$ & $\begin{array}{c}{\left[\mathrm{S}_{\mathrm{III}}\right]} \\
18.71 \mu \mathrm{m}\end{array}$ & $\begin{array}{c}\mathrm{H}_{2} \mathrm{~S}(2) \\
12.28 \mu \mathrm{m}\end{array}$ & $\begin{array}{c}\mathrm{H}_{2} \mathrm{~S}(1) \\
17.04 \mu \mathrm{m}\end{array}$ & $11.3 \mu \mathrm{m} \mathrm{PAH}$ \\
\hline NGC 2369 & $9.7 \times 6.2$ & $<5.5$ & 194. \pm 11 & 15. \pm 3 & 44. \pm 4 & 19. \pm 6 & 51. \pm 2 & 798. \pm 34 . \\
\hline NGC 3110 & $15 \times 8.4$ & $<6.9$ & 164. \pm 14 & $30 . \pm 3$ & $59 . \pm 5$ & $<5.1$ & $33 . \pm 3$ & 877. \pm 29 \\
\hline NGC 3256 & $6.0 \times 3.8$ & 15. \pm 3 & 1229. \pm 48 & 189. \pm 5 & 395. \pm 8 & $65 . \pm 6$ & 124. \pm 3 & 2998. \pm 30 . \\
\hline ESO 320-G030 & $6.3 \times 4.2$ & $<4.8$ & 152. \pm 7 & 12. \pm 2 & 47. \pm 5 & 13. \pm 4 & $21 . \pm 2$ & 677. \pm 29 \\
\hline NGC 5135 & $9.0 \times 5.0$ & 19. \pm 2 & 141. \pm 12 & $66 . \pm 3$ & $39 . \pm 3$ & 14. \pm 4 & 32. \pm 2 & 593. \pm 24 \\
\hline IC $4518 \mathrm{E}$ & $8.0 \times 6.4$ & $<1.6$ & 11. \pm 1 & $0.90 \pm 0.32$ & $3.7 \pm 1.0$ & $<1.0$ & $2.1 \pm 0.5$ & 34. \pm 3 \\
\hline Zw 049.057 & $4.8 \times 4.3$ & $<4.3$ & 23. \pm 3 & $<2.2$ & $<1.4$ & $<6.1$ & $7.9 \pm 1.0$ & 89. \pm 24 \\
\hline IRAS $17138-1017$ & $11.5 \times 5.8$ & $<3.9$ & 159. \pm 9 & $25 . \pm 1$ & 54. \pm 3 & $8.3 \pm 3.7$ & 24. \pm 2 & $505 . \pm 22$ \\
\hline IC 4687 & $11 \times 7.2$ & $10 . \pm 2$ & 198. \pm 10 & 57. \pm 2 & 103. \pm 3 & $8.9 \pm 5.5$ & 22. \pm 1 & 750. \pm 23 \\
\hline NGC 6701 & $8.1 \times 5.4$ & $<14$ & 117. \pm 9 & 16. \pm 3 & 32. \pm 7 & $<8.8$ & 26. \pm 3 & $600 . \pm 14$ \\
\hline NGC 7130 & $16 \times 10$ & $<9.6$ & 158. \pm 14 & 36. \pm 4 & $39 . \pm 7$ & $<7.0$ & $29 . \pm 5$ & 643. \pm 29 \\
\hline IC 5179 & $10 \times 6.9$ & $<9.2$ & $290 . \pm 17$ & $28 . \pm 3$ & $91 . \pm 5$ & 19. \pm 5 & $35 . \pm 6$ & 1334. \pm 29 \\
\hline NGC 7591 & $8.8 \times 6.8$ & $<5.8$ & 54. \pm 5 & $4.4 \pm 2.5$ & $10 . \pm 3$ & $<3.6$ & $10 . \pm 4$ & 266. \pm 13 \\
\hline NGC 7771 & $14 \times 6$ & $<12$ & 148. \pm 11 & $<9.2$ & 41. \pm 9 & $20 . \pm 10$ & 41. \pm 4 & $675 . \pm 23$ \\
\hline
\end{tabular}

Notes. Fluxes are expressed in units of $10^{-17} \mathrm{~W} \mathrm{~m}^{-2}$. The fluxes of Arp 299 (NGC 3690/IC 694) are given in Alonso-Herrero et al. (2009b).

a Approximated projected square size of the aperture in kpc. See Section 3.3 for details.

Table 5

High-resolution (SH) Spectra: Nuclear Spectra

\begin{tabular}{|c|c|c|c|c|c|c|c|c|}
\hline Name & Size $^{a}$ & $\begin{array}{c}{[\mathrm{S} \text { IV }]} \\
10.51 \mu \mathrm{m} \\
\end{array}$ & $\begin{array}{c}{[\mathrm{Ne} \text { II }]} \\
12.81 \mu \mathrm{m} \\
\end{array}$ & $\begin{array}{c}{[\mathrm{Ne} \text { III] }} \\
15.55 \mu \mathrm{m}\end{array}$ & $\begin{array}{c}{\left[\mathrm{S}_{\mathrm{III}}\right]} \\
18.71 \mu \mathrm{m} \\
\end{array}$ & $\begin{array}{c}\mathrm{H}_{2} \mathrm{~S}(2) \\
12.28 \mu \mathrm{m}\end{array}$ & $\begin{array}{c}\mathrm{H}_{2} \mathrm{~S}(1) \\
17.04 \mu \mathrm{m}\end{array}$ & $11.3 \mu \mathrm{m} \mathrm{PAH}$ \\
\hline NGC 2369 & 1.2 & $<0.44$ & $52 . \pm 3$ & $3.4 \pm 0.2$ & $8.6 \pm 0.2$ & $3.1 \pm 0.2$ & $5.5 \pm 0.2$ & $138 . \pm 3$ \\
\hline NGC 3110 & 1.9 & $<0.27$ & 26. \pm 1 & $2.4 \pm 0.1$ & $6.2 \pm 0.2$ & $1.3 \pm 0.3$ & $2.8 \pm 0.1$ & 99. \pm 2 \\
\hline NGC 3256 & 1.1 & $1.8 \pm 0.3$ & $260 . \pm 14$ & 27. \pm 1 & $60 . \pm 1$ & $8.0 \pm 0.8$ & 14. \pm 1 & 489. \pm 5 \\
\hline ESO 320-G030 & 1.2 & $<0.39$ & 41. \pm 2 & $3.3 \pm 0.1$ & 11. \pm 0.1 & $2.8 \pm 0.3$ & $4.0 \pm 0.1$ & 172. \pm 3 \\
\hline NGC 5135 & 1.6 & $8.0 \pm 0.2$ & 51. \pm 3 & 23. \pm 1 & 12. \pm 0.1 & $3.2 \pm 0.3$ & $4.9 \pm 0.2$ & 166. \pm 3 \\
\hline IC $4518 \mathrm{E}$ & 1.8 & $<0.082$ & $1.1 \pm 0.1$ & $0.13 \pm 0.02$ & $0.24 \pm 0.08$ & $0.13 \pm 0.05$ & $0.34 \pm 0.05$ & $5.4 \pm 0.5$ \\
\hline Zw 049.057 & 1.5 & $<0.26$ & $10 . \pm 1$ & $0.63 \pm 0.07$ & $1.8 \pm 0.2$ & $1.4 \pm 0.3$ & $2.2 \pm 0.1$ & 32. \pm 3 \\
\hline IRAS $17138-1017$ & 2.0 & $1.0 \pm 0.2$ & $64 . \pm 3$ & $8.5 \pm 0.2$ & $19 . \pm 1$ & $2.1 \pm 0.6$ & $4.0 \pm 0.4$ & 135. \pm 3 \\
\hline IC 4687 & 2.0 & $2.3 \pm 0.2$ & $55 . \pm 2$ & 12. \pm 0.1 & 23. \pm 0.1 & $2.0 \pm 0.3$ & $3.2 \pm 0.2$ & 189. \pm 3 \\
\hline NGC 6701 & 1.5 & $<0.49$ & 27. \pm 2 & $2.3 \pm 0.1$ & $5.1 \pm 0.2$ & $1.9 \pm 0.2$ & $3.4 \pm 0.2$ & 103. \pm 2 \\
\hline NGC 7130 & 1.9 & $3.6 \pm 0.4$ & $38 . \pm 3$ & 12. \pm 1 & $7.4 \pm 0.6$ & $1.7 \pm 0.8$ & $2.9 \pm 0.3$ & 93. \pm 3 \\
\hline IC 5179 & 1.3 & $<0.39$ & 36. \pm 2 & $2.7 \pm 0.1$ & $8.5 \pm 0.3$ & $1.4 \pm 0.1$ & $2.6 \pm 0.1$ & $120 . \pm 3$ \\
\hline NGC 7591 & 1.9 & $<0.24$ & 16. \pm 1 & $1.3 \pm 0.1$ & $2.4 \pm 0.2$ & $1.1 \pm 0.2$ & $1.8 \pm 0.2$ & 48. \pm 2 \\
\hline NGC 7771 & 1.7 & $<0.38$ & 37. \pm 2 & $3.0 \pm 0.1$ & $6.7 \pm 0.4$ & $3.0 \pm 0.5$ & $4.8 \pm 0.2$ & $115 . \pm 4$ \\
\hline
\end{tabular}

Notes. Fluxes are expressed in units of $10^{-17} \mathrm{~W} \mathrm{~m}^{-2}$. The fluxes of Arp 299 (NGC 3690/IC 694) are given in Alonso-Herrero et al. (2009b).

a Projected square size of the aperture in kpc. The aperture size is $5.5 \times 5$ ".5 for all the galaxies.

or less uniform shallow silicate absorption and relatively high metallicity.

The PAH ratios, $\mathrm{PAH}$ equivalent width (EW), and silicate depth measured in the nuclear and integrated low-resolution spectra are listed in Table 3 . Tables 4 and 5 show, respectively, the integrated and nuclear fine structure line fluxes in the highresolution spectra.

\subsection{Silicate Feature Maps}

Amorphous silicate grains present a broad feature centered at $9.7 \mu \mathrm{m}$ that can be measured from the SL cubes. We used a method similar to that proposed by Spoon et al. (2007) to measure the observed silicate strength. First, we extracted onedimensional spectra from the cube as described in Section 3.1. For each spectrum, we estimated the continuum expected at $10 \mu \mathrm{m}$ by fitting a power law through the feature-free continuum pivots at $5.5 \mu \mathrm{m}$ and $13 \mu \mathrm{m}$. Then we compared the estimated continuum and the measured flux using the following expression:

$$
S_{\mathrm{Si}}=\ln \frac{f_{\text {obs }}(10 \mu \mathrm{m})}{f_{\text {cont }}(10 \mu \mathrm{m})},
$$

where $S_{\mathrm{Si}}$ is the silicate strength. When the silicate strength is negative, the silicate feature is in absorption, whereas a positive silicate strength indicates that the feature is seen in emission.

We estimated the flux at $10 \mu \mathrm{m}$ instead of $9.7 \mu \mathrm{m}$ (the maximum of the feature) to avoid a possible contamination by the molecular hydrogen emission line $\mathrm{H}_{2} \mathrm{~S}(3)$ at $9.67 \mu \mathrm{m}$.

There are several factors that affect the accuracy of the silicate strength measurement. The most important source of uncertainty is the continuum estimate, because of the presence of broad PAH bands in the spectra. We selected continuum pivots similar to those used by Spoon et al. (2007), so that we can make meaningful comparisons with their results. The $S_{\mathrm{Si}}$ is equivalent to the optical depth of the silicate absorption $\left(\tau_{\mathrm{Si}}\right)$ defined in Rieke \& Lebofsky (1985). Alternatively, a more accurate way to measure the silicate optical depth is to fit all the features (PAHs, emission lines, and dust continuum) present in 

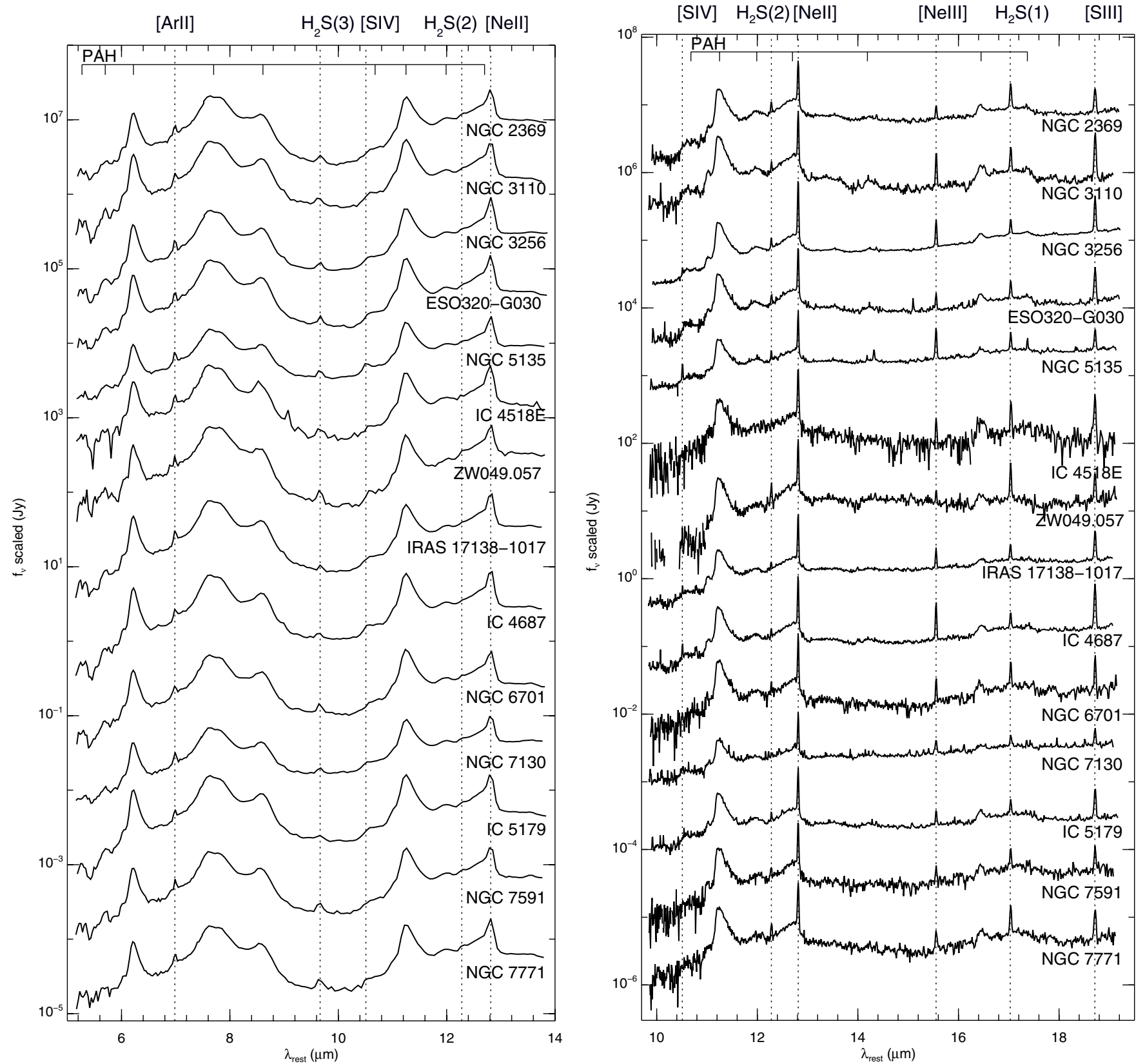

Figure 6. Rest-frame SL (left panel) and SH (right panel) integrated spectra of the sample. The Arp 299 spectra was shown in Alonso-Herrero et al. (2009b). The fluxes have been scaled for better comparison. The scale factors are, starting from the top galaxy, $2 \times 10^{7}, 6 \times 10^{6}, 1.2 \times 10^{5}, 1.5 \times 10^{5}, 2 \times 10^{4}, 3 \times 10^{4}, 7 \times 10^{3}$, $1 \times 10^{2}, 8,1,1 \times 10^{-1}, 1 \times 10^{-2}, 5 \times 10^{-3}, 2 \times 10^{-4}$ for the left panel and $1 \times 10^{7}, 2 \times 10^{6}, 2 \times 10^{4}, 2 \times 10^{3}, 5 \times 10^{3}, 2 \times 10^{2}, 2,2 \times 10^{-1}, 5 \times 10^{-2}, 2 \times 10^{-3}$, $2 \times 10^{-4}, 2 \times 10^{-4}, 1 \times 10^{-5}$ for the right panel.

the spectrum as PAHFIT does (Smith et al. 2007b). However, to avoid ambiguities between the PAH strength and the silicate absorption, the entire low-resolution spectral range $(5.2-38 \mu \mathrm{m})$ is desirable. At longer wavelengths $(>14.5 \mu \mathrm{m})$ the pixel size is about 2.5 times larger than at shorter wavelengths and since we are interested in the spatial distribution of the silicate feature we decided to use the first method to estimate the continuum. To compared these methods, first we fitted the integrated spectra using PAHFIT, assuming a foreground screen dust geometry to obtain the $\tau_{9.7}$, and then we compared this value with the measured $S_{\mathrm{Si}}$. We found that both quantities are well correlated.

Keeping in mind that the main source of uncertainty of the silicate strength measurements is the continuum determination, we estimated that the typical values of the statistical uncertainty are $\sim 0.05$ for the nuclei and less than $\sim 0.15$ for the extranuclear regions. The maps of the silicate feature strength are presented in Figure 8.

\subsection{SH Velocity Fields}

The SH spectra were also used for rough determination of velocities. Figure 9 shows an example of line fitting for selected regions of IC 5179 to illustrate the detection of variations in the line position, even with the relatively low spectral resolution of the $\mathrm{SH}$ module.

Since the spectral resolution, $R \sim 600$, is lower than that of most kinematic studies, we tested the validity of the velocities with synthetic spectra. We created these spectra to simulate an unresolved spectral line as seen by the $\mathrm{SH}$ module and then we 

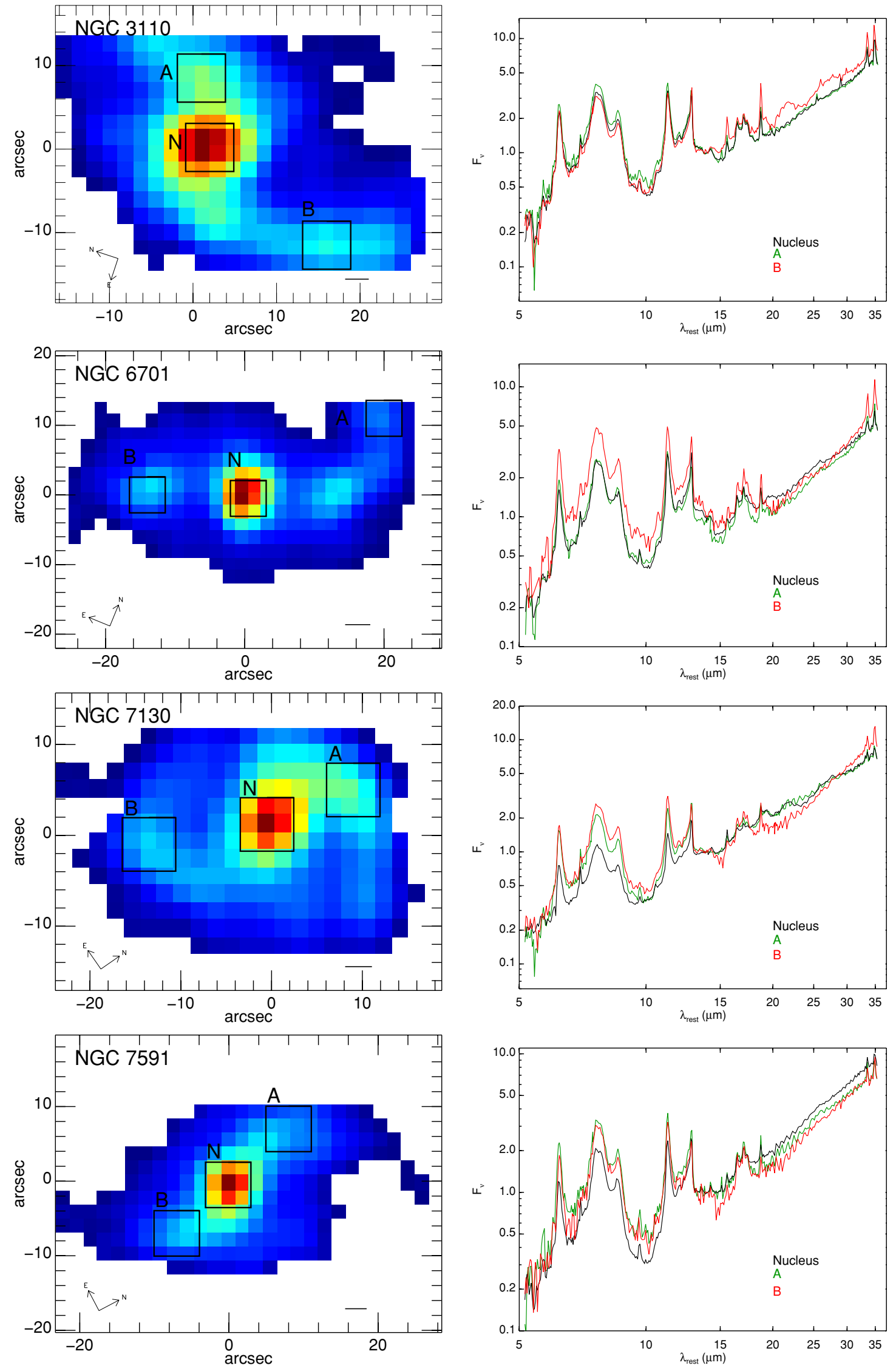

Figure 7. Left: Spitzer/IRS SL spectral maps of the $7.7 \mu \mathrm{m}$ PAH feature. The image orientation is indicated on the maps for each galaxy. The scale represents $1 \mathrm{kpc}$. The black squares are the positions and sizes of the extraction apertures. Right: low-resolution spectra normalized at $14 \mu \mathrm{m}$.

(A color version of this figure is available in the online journal.) 

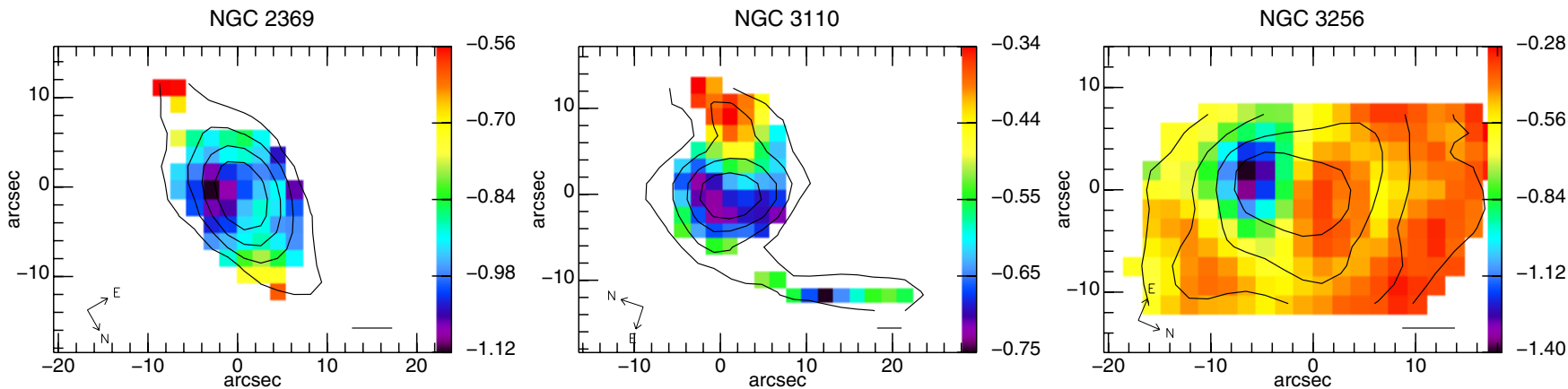

Figure 8. Maps of the $9.7 \mu \mathrm{m}$ silicate feature strength as defined in Section 3.4. The $5.5 \mu \mathrm{m}$ continuum contours are displayed to guide the eye. The image orientation is indicated on the maps for each galaxy. The scale represents $1 \mathrm{kpc}$. The maps are shown in a linear scale.

(A color version and an extended version of this figure are available in the online journal.)

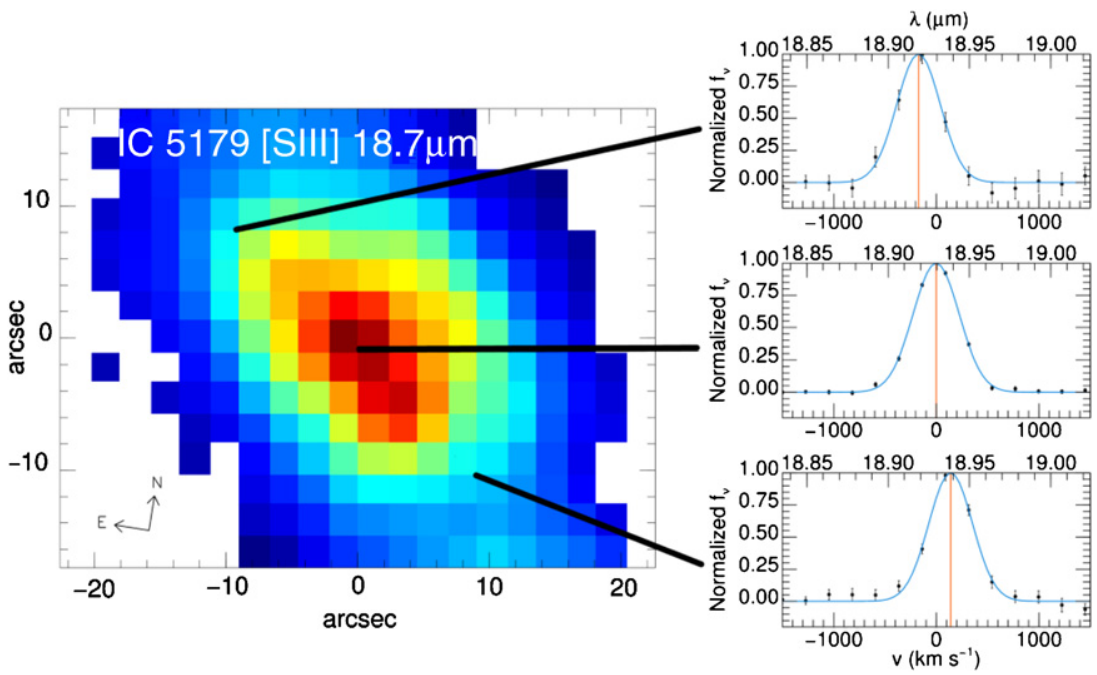

Figure 9. Left panel is the map of the [S III] $18.71 \mu \mathrm{m}$ line flux of IC 5179. The right panels show the fit to this line for three selected regions of this galaxy.

(A color version of this figure is available in the online journal.)

tried to recover the original line parameters. The EW of the simulated lines was similar to the observed ones. We added noise to the spectra to match the continuum $\mathrm{S} / \mathrm{N}$ of the maps. The continuum $\mathrm{S} / \mathrm{N}$, for most of the galaxies, is in the range $\sim 60-100$ at the nuclei and $\sim 3-10$ at the external regions. To allow for the effect of the telescope pointing uncertainties, we distorted the wavelength scale by $\sim 5 \%$ (estimated from the pointing information in the BCD file headers). We found that this effect limits the accuracy of the velocity determination to $\pm 10 \mathrm{~km} \mathrm{~s}^{-1}$, independent of the $\mathrm{S} / \mathrm{N}$ of the spectra. For extended sources, which fill uniformly the slit, the wavelengths are more stable, and then the uncertainty due to the pointing inaccuracy would be lower. The galaxies studied here are not point sources, neither uniformly extended sources, thus this uncertainty estimation is an upper limit. Including the uncertainty in the absolute wavelength calibration (equivalent to $10 \%$ of a pixel), we found the minimum uncertainty to be $\pm 20 \mathrm{~km} \mathrm{~s}^{-1}$. We therefore conclude that the uncertainty in the velocity field is in the range of $10-30 \mathrm{~km} \mathrm{~s}^{-1}$, depending on the pointing accuracy achieved and the $\mathrm{S} / \mathrm{N}$ of an individual spectrum. Thus, we consider that variations in the velocity maps larger than $\sim 20 \mathrm{~km} \mathrm{~s}^{-1}$ are likely to be real. Figure 10 shows the velocity fields derived for the [Ne II] $12.81 \mu \mathrm{m}$ and $\mathrm{H}_{2} \mathrm{~S}(1)$ emission lines; they range up to a total gradient of $\sim 200 \mathrm{~km} \mathrm{~s}^{-1}$ and hence include a substantial amount of information about the sources.

\section{THE SILICATE FEATURE}

\subsection{Spatially Resolved Measurements}

LIRGs are known to contain highly obscured regions $\left(\mathrm{A}_{V} \simeq\right.$ 4-50 mag, see, e.g., Veilleux et al. 1995; Genzel et al. 1998; Alonso-Herrero et al. 2000, 2006), usually coincident with the nuclei of the galaxies. Assuming an extinction law and a dust distribution geometry the silicate strength can be converted into a visual extinction.

The maps of the silicate feature of our sample are shown in Figure 8. In a large fraction of the systems (80\%) the most obscured regions appear to be coincident with the nuclei. In other cases, the nuclear regions appear to be slightly less obscured than the surrounding regions (e.g., NGC 7130) or they show a complicated morphology (e.g., IRAS 17138-1017, NGC 7771, IC 4687). We note that the spectra used for measuring the strength of the silicate feature are averaged over $\sim 1 \mathrm{kpc}$ scales, and thus some of the real variations seen on smaller scales using ground-based mid-IR observations are smoothed out (e.g., the highly obscured southern nucleus of NGC 3256, see DíazSantos et al. 2008).

Figure 11 shows the distribution of the silicate strengths measured from the spatially resolved maps for all the galaxies. They are in the range $\sim 0$ to $\sim-2.0$, with a median of -0.61 . The strengths of the majority of the LIRGs are moderate 


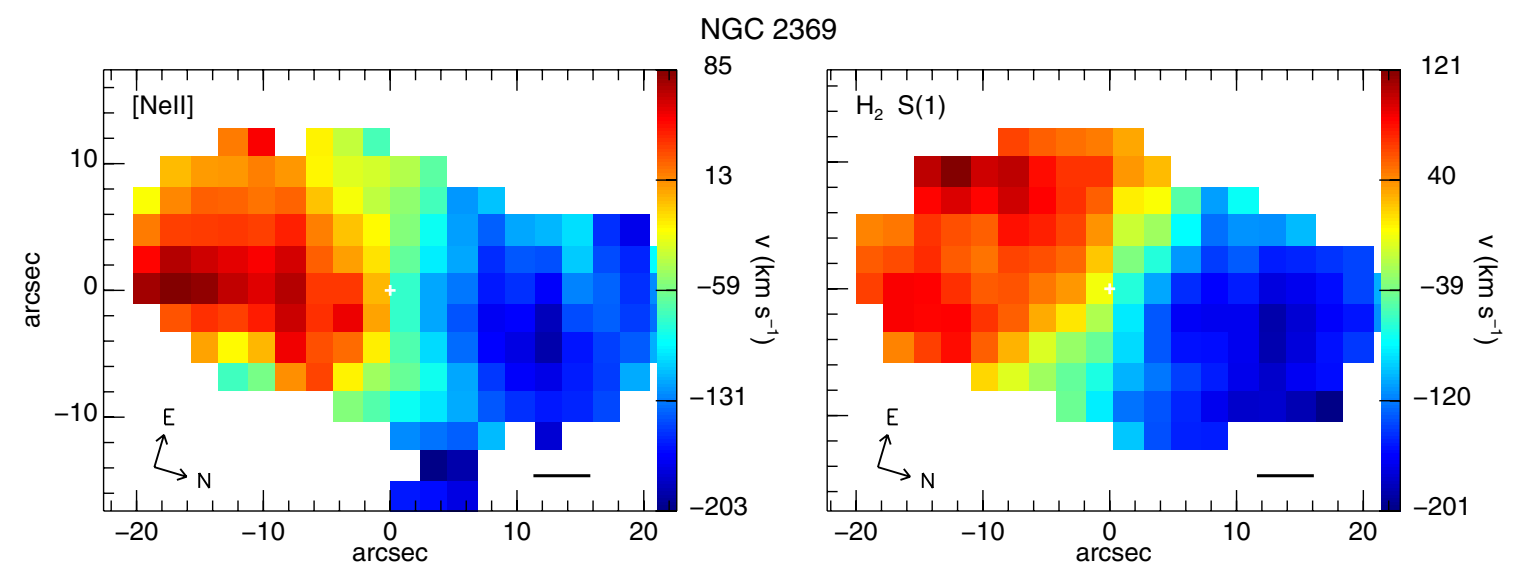

Figure 10. Maps of the observed velocity field of [Ne II] $12.81 \mu \mathrm{m}$ and $\mathrm{H}_{2} \mathrm{~S}(1)$ at $17.0 \mu \mathrm{m}$. The white cross marks the coordinates of the nucleus as listed in Table 1 . The image orientation is indicated on the maps for each galaxy. The scale represents $1 \mathrm{kpc}$.

(A color version and an extended version of this figure are available in the online journal.)

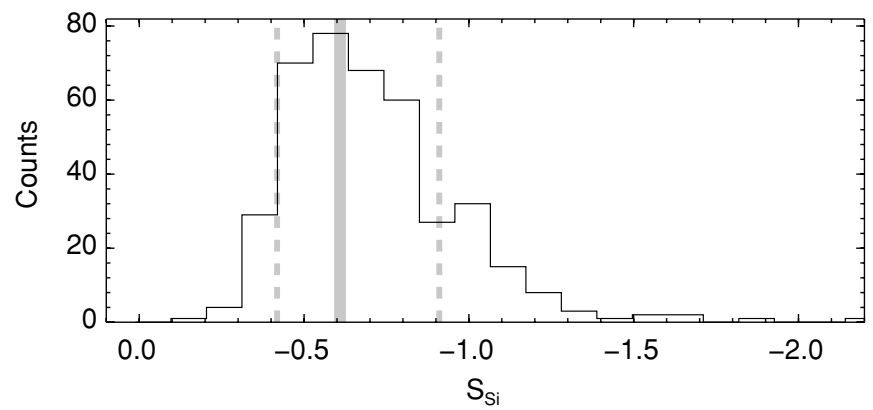

Figure 11. Histogram of the spatially resolved measurements of the silicate strength for all galaxies measured in boxes of $2 \times 2$ pixels. The solid gray line indicates the median silicate strength. The dashed gray lines define the range including $68 \%$ of the data points.

$\left(S_{\mathrm{Si}} \sim-0.4\right.$ to -0.9$)$, and intermediate between those observed in starburst galaxies (Brandl et al. 2006) and ULIRGs (Spoon et al. 2007; Sirocky et al. 2008).

If we assume a foreground screen of dust, the silicate absorption strength can be expressed in terms of the optical extinction $\left(A_{V}\right)$ using the conversion factor $A_{V} / S_{\mathrm{Si}}=16.6$ (Rieke \& Lebofsky 1985). We then find that the median $A_{V}$ of the spatially resolved measurements is $\sim 10 \mathrm{mag}$. The most extincted region in our sample of LIRGs is the nuclear region of IC 694, also known as Arp 299-A (see also Alonso-Herrero et al. 2009b), with $A_{V} \sim 37 \mathrm{mag}$. The foreground screen assumption gives the minimum extinction compatible with the measurements. Most of the spatially resolved measurements $(68 \%)$ are in the range of -0.4 to -0.9 . This range is much narrower than that observed in the nuclear regions of ULIRGs $\left(0>S_{\mathrm{Si}}>-4\right.$; Spoon et al. 2007). The similarity of the silicate absorption strengths among our sample members suggests that they may be in part a product of radiative transfer in dusty regions. We can approximate this possibility by assuming that the dust and emission sources are mixed. The resulting median $\mathrm{A}_{V}$ is then $\sim 20 \mathrm{mag}$, about 2 times greater than for the foreground screen geometry.

The nuclear and integrated values of the silicate strength are listed in Table 3. For all the galaxies, we find that the integrated strength of the silicate feature is smaller than, although comparable to, the nuclear one. The nuclear silicate strengths range from -0.4 to -1.9 , although most of them are larger than -1.0 . The integrated strength is $-0.3<S_{\mathrm{Si}}<-1.2$, somewhat shallower than the nuclear strength. The LIRGs with the largest variations between the nuclear and integrated silicate features are NGC 3256, Arp 299, NGC 7130, and NGC 7591.

\subsection{The $S_{S i}$ versus $6.2 \mu \mathrm{m}$ PAH EW Diagram}

Spoon et al. (2007) presented a diagram using the EW of $6.2 \mu \mathrm{m}$ PAH feature and the silicate strength to provide a general classification of infrared galaxies. ULIRGs appear distributed along two branches. The horizontal branch is populated by unobscured AGNs (QSO and Sy1) in the left-hand side of the diagram (class 1A) and by starburst galaxies in the righthand side (class 1C) of the diagram. The diagonal branch goes from deeply obscured nuclei (class 3A) to unobscured starbursts (class 1A).

In Figure 12, we present a similar diagram for our sample of LIRGs. The left panel shows the spatially resolved measurements of our sample of LIRGs. Most of them appear in this diagram at the region where both branches intercept. The nuclear and surrounding regions of galaxies classified as Seyferts using optical spectroscopy (NGC 5135: Sy2, NGC 7130: LINER/Sy and Arp 299-B1: Sy2, see Table 1) are located in the 1B region (intermediate between AGN and starbursts), in agreement with their well-known composite nature. NGC 6701 and NGC 7591 are classified as composite objects which is likely to be a combination of an AGN and star formation activity (see AlonsoHerrero et al. 2009a). Both nuclei appear quite close to region 1B. The two most obscured nuclei in the sample, Arp 299-A and the southern nucleus of NGC 3256, and their neighboring regions, are in the upper part of the diagonal branch populated by ULIRGs, although they do not reach the silicate strengths of the deeply embedded ULIRG nuclei.

The comparison between the nuclear and integrated values (the right panel of Figure 12) shows that the integrated spectra tend to have lower silicate strengths and larger $6.2 \mu \mathrm{m}$ PAH EWs. We find that on average the integrated $6.2 \mu \mathrm{m}$ PAH $\mathrm{EW}$ is $\sim 30 \%$ larger than the nuclear values, whereas the integrated silicate strength is $\sim 15 \%$ lower than that found in the nuclei. That is, the integrated values move the galaxies to the pure starburst class region $(1 \mathrm{C})$ in this diagram. In the case of all the active galaxies of the sample, the nucleus is not sufficiently bright as to dominate the integrated mid-infrared spectrum and the dominant contribution from star formation, which is extended over several kpc (see Alonso-Herrero et al. 

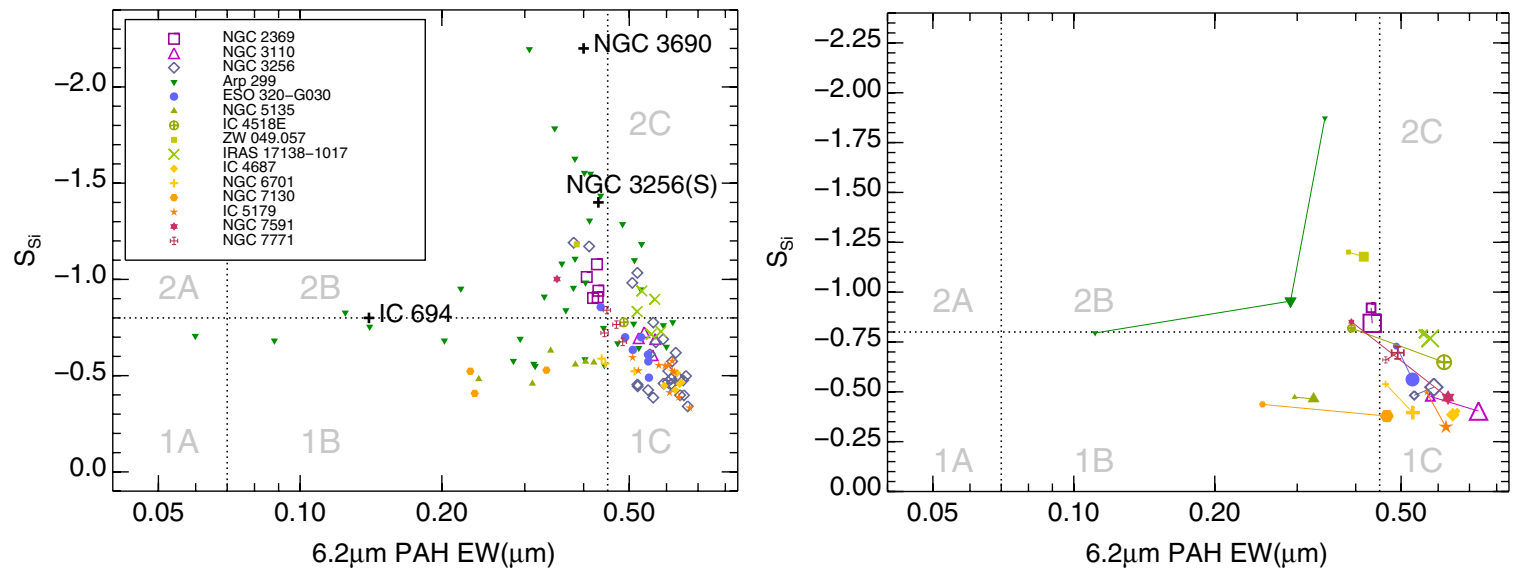

Figure 12. $S_{\mathrm{Si}}$ vs. the $6.2 \mu \mathrm{m}$ PAH equivalent width (EW) diagram, similar to that presented by Spoon et al. (2007). Left: spatially resolved measurements for our sample of LIRGs using a $2 \times 2$ pixel box (3".7 × 3.'7 aperture). The black crosses are the two nuclei of Arp 299 (IC 694 and NGC 3690) and the southern nucleus of NGC 3256. Right: nuclear $\left(5^{\prime \prime} .5 \times 55^{\prime \prime} 5\right.$ aperture) and integrated values of each galaxy. The small symbols correspond to the nuclear value and the large symbols to the integrated galaxy. Nuclear and integrated are connected by a line to guide the eye. In both panels, following Spoon et al. (2007), the parameter space is divided into six classes out of their nine classes. Class 1A is populated by unobscured AGNs. They have featureless spectra with a shallow silicate feature. Class 1C corresponds to pure starbursts (PAH dominated spectra). The spectra of a galaxy in the class $1 \mathrm{~B}$ are intermediate between the AGN spectra and the pure starburst. Classes $2 \mathrm{~B}$ and $2 \mathrm{C}$ have a deeper silicate absorption, so do classes 3A and 3B (not plotted here). The majority of ULIRGs not dominated by an AGN lie in these classes.

(A color version of this figure is available in the online journal.)

Table 6

Nuclear Flux/Integrated Flux

\begin{tabular}{|c|c|c|c|c|c|c|c|}
\hline \multirow[t]{2}{*}{ Name } & \multicolumn{3}{|c|}{ Central $2.0 \mathrm{kpc} /$ Integrated Flux } & \multicolumn{4}{|c|}{ Central $2.0 \mathrm{kpc} /$ Integrated flux } \\
\hline & $\begin{array}{c}5.5 \mu \mathrm{m} \\
\text { Continuum } \\
\end{array}$ & $6.2 \mu \mathrm{m}$ PAH & $11.3 \mu \mathrm{m}$ PAH & $\begin{array}{c}15 \mu \mathrm{m} \\
\text { Continuum } \\
\end{array}$ & {$[\mathrm{Ne} \mathrm{II}] 12.81 \mu \mathrm{m}$} & {$[\mathrm{Ne}$ III $] 15.56 \mu \mathrm{m}$} & $\mathrm{H}_{2} \mathrm{~S}(1)$ \\
\hline NGC 2369 & $0.41 \pm 0.02$ & $0.41 \pm 0.01$ & $0.32 \pm 0.01$ & $0.39 \pm 0.02$ & $0.36 \pm 0.02$ & $0.36 \pm 0.02$ & $0.20 \pm 0.02$ \\
\hline NGC 3110 & $0.13 \pm 0.05$ & $0.12 \pm 0.02$ & $0.10 \pm 0.01$ & $0.13 \pm 0.01$ & $0.07 \pm 0.03$ & $0.12 \pm 0.02$ & $0.08 \pm 0.03$ \\
\hline NGC 3256 & $0.38 \pm 0.01$ & $0.36 \pm 0.01$ & $0.34 \pm 0.01$ & $0.39 \pm 0.01$ & $0.31 \pm 0.01$ & $0.41 \pm 0.01$ & $0.20 \pm 0.03$ \\
\hline ESO 320-G030 & $0.57 \pm 0.02$ & $0.54 \pm 0.01$ & $0.49 \pm 0.01$ & $0.48 \pm 0.01$ & $0.49 \pm 0.02$ & $0.40 \pm 0.01$ & $0.38 \pm 0.02$ \\
\hline NGC 5135 & $0.45 \pm 0.01$ & $0.42 \pm 0.01$ & $0.35 \pm 0.01$ & $0.39 \pm 0.01$ & $0.36 \pm 0.01$ & $0.28 \pm 0.01$ & $0.19 \pm 0.03$ \\
\hline IC $4518 \mathrm{E}$ & $0.21 \pm 0.10$ & $0.18 \pm 0.10$ & $0.15 \pm 0.03$ & $0.14 \pm 0.02$ & $\ldots$ & $0.15 \pm 0.01$ & $0.19 \pm 0.06$ \\
\hline Zw 049.057 & $0.53 \pm 0.08$ & & $0.50 \pm 0.02$ & $0.52 \pm 0.02$ & & $0.47 \pm 0.02$ & \\
\hline IRAS $17138-1017$ & $0.31 \pm 0.03$ & $0.31 \pm 0.02$ & $0.25 \pm 0.01$ & $0.31 \pm 0.01$ & $0.27 \pm 0.01$ & $0.19 \pm 0.01$ & $0.15 \pm 0.05$ \\
\hline IC 4687 & $0.24 \pm 0.03$ & $0.25 \pm 0.01$ & $0.23 \pm 0.01$ & $0.19 \pm 0.01$ & $0.17 \pm 0.01$ & $0.15 \pm 0.01$ & $0.13 \pm 0.05$ \\
\hline NGC 6701 & $0.25 \pm 0.03$ & $0.23 \pm 0.02$ & $0.20 \pm 0.02$ & $0.22 \pm 0.01$ & $0.16 \pm 0.02$ & $0.24 \pm 0.01$ & $0.15 \pm 0.03$ \\
\hline NGC 7130 & $0.28 \pm 0.02$ & $0.13 \pm 0.03$ & $0.14 \pm 0.02$ & $0.14 \pm 0.01$ & $0.21 \pm 0.03$ & $0.10 \pm 0.01$ & $0.09 \pm 0.05$ \\
\hline IC 5179 & $0.23 \pm 0.03$ & $0.20 \pm 0.01$ & $0.18 \pm 0.01$ & $0.19 \pm 0.01$ & $0.16 \pm 0.02$ & $0.11 \pm 0.01$ & $0.13 \pm 0.03$ \\
\hline NGC 7591 & $0.22 \pm 0.05$ & $0.14 \pm 0.05$ & $0.13 \pm 0.02$ & $0.22 \pm 0.01$ & $0.23 \pm 0.03$ & $0.23 \pm 0.01$ & $0.17 \pm 0.04$ \\
\hline NGC 7771 & $0.24 \pm 0.02$ & $0.28 \pm 0.02$ & $0.23 \pm 0.01$ & $0.24 \pm 0.01$ & $0.20 \pm 0.03$ & $0.23 \pm 0.01$ & $0.12 \pm 0.04$ \\
\hline
\end{tabular}

2006, 2009b, 2009a) makes the integrated spectrum look more starburst-like.

\section{FINE STRUCTURE EMISSION LINES}

\subsection{Morphology}

Although the limited spatial resolution $(\sim 1 \mathrm{kpc})$ of the $\mathrm{SH}$ maps does not allow us to study the galaxy morphologies in great detail, it is possible to analyze general trends. The exact spatial distribution of the emission lines depends on the physical conditions and the age of the dominating stellar population in each region. In Appendix B we discuss the galaxies individually.

Figure 4 shows the SH spectral maps of the most prominent features, as well as those of the $15 \mu \mathrm{m}$ continuum. The [Ne II] $12.81 \mu \mathrm{m}$, [Ne III] $15.56 \mu \mathrm{m}$, and [S III] $18.71 \mu \mathrm{m}$ fine structure lines have an overall morphology similar to that of the $15 \mu \mathrm{m}$ continuum. We detect [S IV] $10.51 \mu \mathrm{m}$ emission in only five systems. In two of them (NGC 7130 and NGC 5135), the [S IV] $10.51 \mu \mathrm{m}$ emission mainly comes from the active nuclei (Díaz-Santos et al. 2010). Our data do not allow us to deter- mine whether there is a low surface brightness [S IV] $10.51 \mu \mathrm{m}$ emission, associated with $\mathrm{H}$ II regions, in these two galaxies due to the relatively low flux of this line. In the other three cases, NGC 3256, IC 4687, and Arp 299 (for the last galaxy, see Alonso-Herrero et al. 2009b), the emission is more extended and we attribute it to the $\mathrm{H}$ II regions seen in the $\mathrm{Pa} \alpha$ and/or $\mathrm{H} \alpha$ images.

We find that the neon emission ([Ne II] $12.81 \mu \mathrm{m}$ and [Ne III] $15.56 \mu \mathrm{m})$ and the $15 \mu \mathrm{m}$ continuum are spatially resolved. We calculated the ratio between the nuclear (circular aperture of radius $2 \mathrm{kpc}$; depending on the distance the aperture radius varies between 1.5 and 3 pixels $)^{8}$ and the integrated neon line emission and $15 \mu \mathrm{m}$ continuum emission for our galaxies, we also used the uncertainty maps to estimate the statistical uncertainty of these ratios (Table 6). On average the uncertainty of the ratio is $\sim 6 \%$. These are upper limits to the relative contribution of the nucleus to the total emission of the galaxy as

\footnotetext{
8 The physical size of the nuclear aperture was chosen so it corresponds to
} the minimum spatial resolution of the most distant galaxy in the sample. 

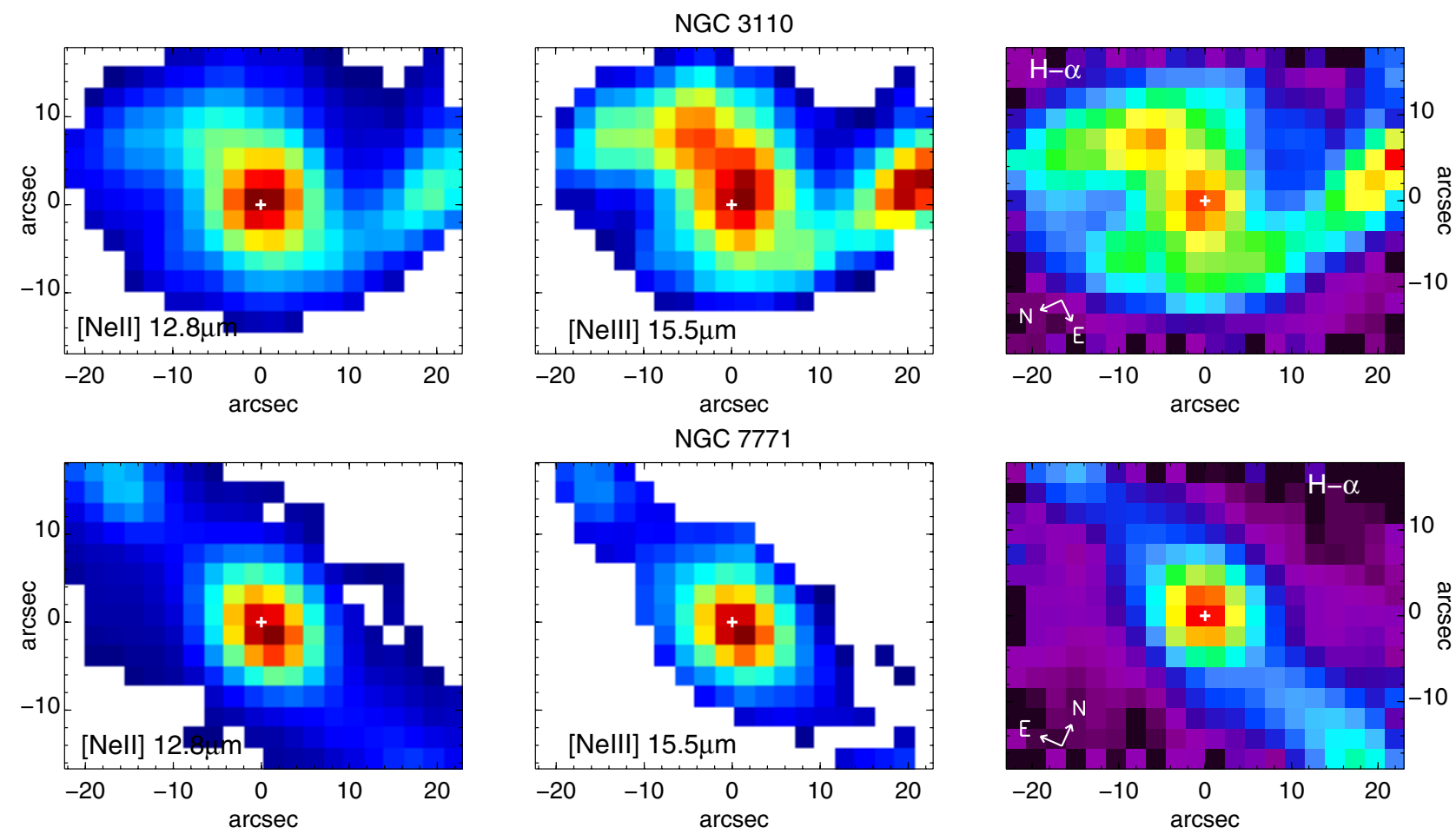

Figure 13. Comparison of the [Ne II] $12.81 \mu \mathrm{m}$ and [Ne III] $15.56 \mu \mathrm{m}$ maps with $\mathrm{H} \alpha$ images from Hattori et al. (2004) for NGC 3110 and NGC 7771 . The image orientation for each galaxy is shown on the $\mathrm{H} \alpha$ image. All the maps are shown in a square root scale.

(A color version of this figure is available in the online journal.)

in some cases the IRS maps do not cover the full extent of the galaxy. In general, the emission arising from the central $2 \mathrm{kpc}$ accounts for less than $50 \%$ of the total emission for the neon lines and the continuum, indicating that the star formation is extended over several $\mathrm{kpc}$. We note however that the LIRGs in this work were selected because they show extended Pa $\alpha$ emission (Section 2.1), and thus, this result needs to be confirmed for a complete sample of LIRGs.

The combined luminosities of the $[\mathrm{Ne}$ II] $12.81 \mu \mathrm{m}$ and [Ne III] $15.56 \mu \mathrm{m}$ lines are good tracers of the star formation rate in galaxies (Ho \& Keto 2007), so a good morphological correspondence is expected between them and $\mathrm{H} \alpha$. To further explore the behavior of the neon emission, Figure 13 compares the IRS neon spectral maps with $\mathrm{H} \alpha$ images $^{9}$ of Hattori et al. (2004) for NGC 3110 and NGC 7771. The SH and $\mathrm{H} \alpha$ maps of NGC 3110 show emission from the nuclear region and from $\mathrm{H}$ II regions in the spiral arms. The [Ne III] $15.56 \mu \mathrm{m}$ emission (also the [S III]18.71 $\mu \mathrm{m}$ emission) from these H II regions is comparable to the emission arising from the nucleus, while [Ne II] $12.81 \mu \mathrm{m}$ is more concentrated around the nucleus. We note, however, that the $\mathrm{H} \alpha$ image is not corrected for extinction. This is especially important in the nuclear region $\left(S_{\mathrm{Si}} \sim-0.48\right.$, see Table $3, A_{V} \sim 8 \mathrm{mag}$ ) as it is the most extincted region in this galaxy. For NGC 7771, the [Ne II] $12.81 \mu \mathrm{m}$ [Ne III] $15.56 \mu \mathrm{m}$ and $\mathrm{H} \alpha$ emissions show similar morphologies, both in the nuclear ring of star formation (seen in the Pa $\alpha$ image, Figure 4, but not resolved by the IRS spectral maps), and the extranuclear H II regions, as well as in the diffuse regions between them. These two examples illustrate that at least from a qualitative point of view and on the scales probed here, the $\mathrm{H} \alpha$, [Ne II] $12.81 \mu \mathrm{m}$, and $[\mathrm{Ne}$ III] $15.56 \mu \mathrm{m}$ lines are tracing the same young ioniz-

\footnotetext{
9 The $\mathrm{H} \alpha$ images were degraded to the Spitzer/IRS SH angular resolution, pixel size, and orientation.
}

ing stellar populations (see Ho \& Keto 2007 for a quantitative assessment).

\subsection{Spatially Resolved Line Ratios}

\subsubsection{The [Ne III] $15.56 \mu \mathrm{m} /[\mathrm{Ne}$ II] $12.81 \mu \mathrm{m}$ Line Ratios}

The spectral maps of the [Ne III] $15.56 \mu \mathrm{m} /[\mathrm{Ne}$ II] $12.81 \mu \mathrm{m}$ line ratios for our sample of LIRGs are shown in Figure 5. In most cases the nuclei have the lowest [Ne III] $15.56 \mu \mathrm{m} /$ $[\mathrm{Ne}$ II $] 12.81 \mu \mathrm{m}$ ratios, while the circumnuclear regions and H II regions tend to show higher ratios (see also Figure 14). With the exception of three cases with an active nucleus (NGC 3690, NGC 5135, and NGC 7130), the nuclear values of the $\left[\mathrm{Ne}{ }_{\mathrm{III}}\right] 15.56 \mu \mathrm{m} /\left[\mathrm{Ne} \mathrm{II}_{\mathrm{II}}\right] 12.81 \mu \mathrm{m}$ ratio are all in the range of $0.06-0.10$ (with three exceptions near 0.2, IC 694, IRAS 17138-1017, and IC 4687-see Table 5). Excluding the galaxies hosting an AGN, the median nuclear ratio is 0.08 . The extranuclear values are between 0.06 and 1 , while the integrated values (see Table 4) are between 0.08 and 0.5 . Thornley et al. (2000) find a similar range for the integrated ratios for an additional four LIRGs. This ratio ranges from 0.02 to 6 in starburst galaxies (Verma et al. 2003; Dale et al. 2006, 2009). For the $\mathrm{H}$ II galaxies, the nuclear [Ne III] $15.56 \mu \mathrm{m} /[\mathrm{Ne}$ II $] 12.81 \mu \mathrm{m}$ ratio is a factor $\sim 2-3$ smaller than those measured in the extranuclear regions.

A similar behavior of increasing [Ne III] $15.56 \mu \mathrm{m} /[\mathrm{Ne}$ II] $12.81 \mu \mathrm{m}$ ratios with increasing galactocentric distances has been observed in the nearby galaxies NGC 253 (Devost et al. 2004) and M82 (Beirão et al. 2008), although the physical scales probed are different $(\sim 80 \mathrm{pc}$ in these two galaxies, $\sim 1 \mathrm{kpc}$ in our sample of LIRGs). Galactic H II regions follow the same trend, with $\mathrm{H}$ II regions at increasing distances from the galactic nucleus showing larger [Ne III] $15.56 \mu \mathrm{m} /[\mathrm{Ne}$ II $] 12.81 \mu \mathrm{m}$ ratios (Giveon et al. 2002). 


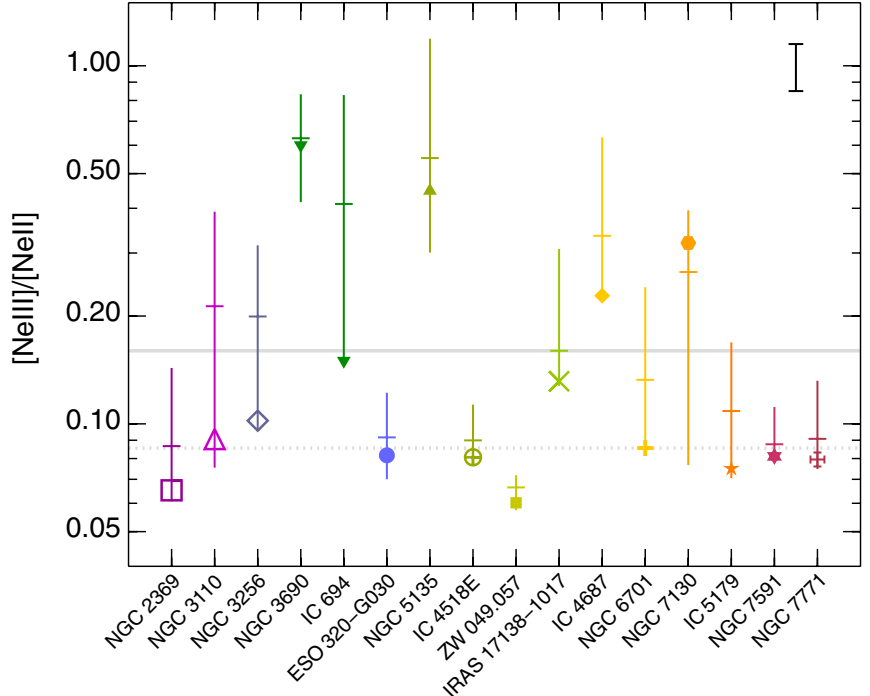

Figure 14. Range of the observed [Ne III $] 15.56 \mu \mathrm{m} /[\mathrm{Ne}$ II $] 12.81 \mu \mathrm{m}$ ratio from the spectral maps. The galaxy symbol (as in Figure 12) indicates the value of the ratio in the nucleus. The horizontal mark is the median of the ratio of each galaxy. The solid gray line is the median of the medians and the dotted line is the median of the nuclear ratios. The black line indicates the typical uncertainty of the ratios.

(A color version of this figure is available in the online journal.)

Note that we did not correct this ratio for extinction. This correction however, only produces variations in the ratio of $5 \%-15 \%$ for the typical extinction of our sample $\left(S_{\mathrm{Si}} \sim-0.6\right.$, that is $\mathrm{A}_{V} \sim 10 \mathrm{mag}$ ). The correction (positive or negative) depends on the adopted extinction law (see Farrah et al. 2007).

The strength of $[\mathrm{Ne} \mathrm{III}] 15.56 \mu \mathrm{m}$ relative to $[\mathrm{Ne}$ II $] 12.81 \mu \mathrm{m}$ decreases with increasing metallicity, increasing age of the stellar population, and increasing nebular density (e.g., Snijders et al. 2007). In accordance with the luminosity- and massmetallicity relations, LIRGs are characterized by uniformly relatively high metallicity (in many cases super-solar), with some indications of slightly (factor of two) reduced levels in their nuclei (Rupke et al. 2008). The Snijders et al. (2007) models predict a $\sim 3$ times larger [Ne III] $15.56 \mu \mathrm{m} /[\mathrm{Ne}$ II] $12.81 \mu \mathrm{m}$ ratio for a given age and density as the metallicity decreases from super-solar $\left(2 Z_{\odot}\right)$ to solar. Thus, metallicity-related effects are unlikely to be the dominant cause of the relative increase of [Ne III] $15.56 \mu \mathrm{m}$ strength outside the central regions of these galaxies. They may in fact to be overcome by some stronger mechanism.

The trend with stellar population age is strong. The expected [Ne III] $15.56 \mu \mathrm{m} /[\mathrm{Ne}$ II $] 12.81 \mu \mathrm{m}$ ratio of an instantaneous burst of star formation falls below $10^{-3}$ for ages $>6 \mathrm{Myr}$ (Rigby \& Rieke 2004). Thornley et al. (2000) showed that the range of the $[\mathrm{Ne}$ III] $15.56 \mu \mathrm{m} /[\mathrm{Ne}$ II $] 12.81 \mu \mathrm{m}$ ratio in starburst galaxies is compatible with models for bursts of star formation. However, from their models and those of Rigby \& Rieke (2004), the nuclear values for LIRGs are below the range that would be expected from such star-forming episodes. The observed nuclear ratios could only be explained in terms of the stellar population if (1) the IMF is truncated at $\sim 30 M_{\odot}$ in the LIRG nuclei (Thornley et al. 2000, Figure 6); or (2) the star formation rate in all the LIRG nuclei has decreased rapidly over the past 10-20 Myr (which seems contrived).

Another explanation is that due to the high densities in the nuclear regions the $[\mathrm{Ne} \mathrm{III}] 15.56 \mu \mathrm{m} /[\mathrm{Ne} \mathrm{II}] 12.81 \mu \mathrm{m}$ ratio is suppressed, including the possibility that a fraction of the most

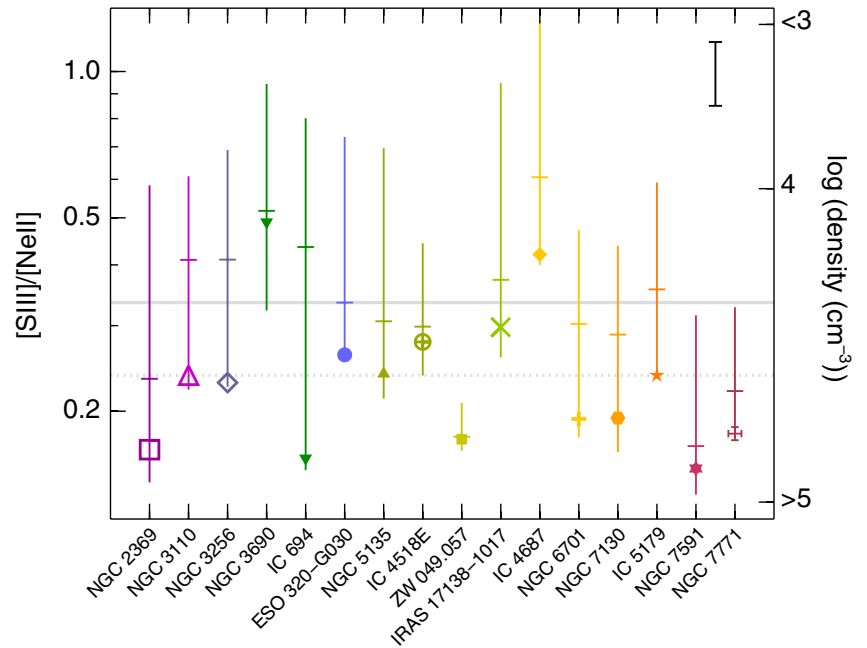

Figure 15. Range of the observed [S III $] 18.71 \mu \mathrm{m} /[\mathrm{Ne}$ II $] 12.81 \mu \mathrm{m}$ ratio from the spectral maps. The symbols are as in Figure 14. The density label corresponds to the $[\mathrm{S}$ III $] 18.71 \mu \mathrm{m} /[\mathrm{Ne} \mathrm{II}] 12.81 \mu \mathrm{m}$ ratio predicted by the Snijders et al. (2007) models for solar metallicity, $q=1.6 \times 10^{8}$ and age $=5$ Myr. The $[\mathrm{Ne}$ III $] 15.56 \mu \mathrm{m} /[\mathrm{Ne}$ II $] 12.81 \mu \mathrm{m}$ ratio predicted using these parameters is $\sim 0.1-0.2$ which is in agreement with the observed ratio.

(A color version of this figure is available in the online journal.)

massive stars are hidden in ultra-compact H II regions (Rigby $\&$ Rieke 2004). The [Ne III $] 15.56 \mu \mathrm{m} /[\mathrm{Ne}$ II $] 12.81 \mu \mathrm{m}$ ratio decreases by approximately a factor of 2 if the density increases from $10^{3}$ to $10^{5} \mathrm{~cm}^{-3}$ (Snijders et al. 2007). We explore the density effects on the neon line ratio in the following section.

Before doing so, we point out that a few galaxies (NGC 3690, NGC 5135, and NGC 7130) have an opposite trend in that the $[\mathrm{Ne}$ III $] 15.56 \mu \mathrm{m} /[\mathrm{Ne}$ II $] 12.81 \mu \mathrm{m}$ ratio becomes larger in their nuclei. We attribute this behavior to the presence of active nuclei. These three galaxies are the only ones classified as Seyfert type and it is likely that the extra hard-ionizing radiation from the AGN is responsible for the high, three times larger than the median, [Ne III]15.56 $\mu \mathrm{m} /[\mathrm{Ne}$ II $] 12.81 \mu \mathrm{m}$ nuclear ratio.

\subsubsection{The $[S$ III] $18.71 \mu \mathrm{m} /[\mathrm{Ne}$ II] $12.81 \mu \mathrm{m}$ Line Ratios}

The excitation potentials of the $[\mathrm{Ne}$ II $] 12.81 \mu \mathrm{m}$ and [S III] $18.71 \mu \mathrm{m}$ emission lines are $21.6 \mathrm{eV}$ and $23.3 \mathrm{eV}$, respectively. Therefore, the $[\mathrm{S} \mathrm{III]}] 18.71 \mu \mathrm{m} /[\mathrm{Ne}$ II $] 12.81 \mu \mathrm{m}$ ratio is almost insensitive to the hardness of the radiation field. On the other hand, this ratio is a good tracer of density in the range $10^{4}-10^{6} \mathrm{~cm}^{-3}$, with lower [S III] $18.71 \mu \mathrm{m} /[\mathrm{Ne}$ II $] 12.81 \mu \mathrm{m}$ ratios indicating larger electron densities (Snijders et al. 2007).

Our spatially resolved measurements show that this ratio ranges from $\sim 0.1$ to $\sim 1.25$ (Figure 15). Assuming a $5 \mathrm{Myr}$ old stellar population, an intermediate ionization parameter $\left(q=1.6 \times 10^{8}\right)$ and solar metallicity (see caption of Figure 15$)$, these ratios correspond to electron densities between $10^{3}$ and $10^{5} \mathrm{~cm}^{-3}$ (Snijders et al. 2007). Like the [Ne III] $15.56 \mu \mathrm{m} /$ $[\mathrm{Ne}$ II $] 12.81 \mu \mathrm{m}$ ratio, for a given galaxy the lowest values of the $[\mathrm{S} \mathrm{III}] 18.71 \mu \mathrm{m} /[\mathrm{Ne}$ II $] 12.81 \mu \mathrm{m}$ ratio occur in the nuclei (in most cases the nuclear value is between $\sim 0.13$ and 0.25 ), while higher ratios are found around them (Figure 5). This indicates, as expected, that the nuclei are denser than the extranuclear $\mathrm{H}$ II regions. According to this spatial distribution the integrated ratios $(\sim 0.2-0.5)$ are $\sim 30 \%$ larger than the nuclear ratios.

The left panel of Figure 16 shows the $[\mathrm{S}$ III] $18.71 \mu \mathrm{m} /[\mathrm{Ne}$ II] $12.81 \mu \mathrm{m}$ ratio versus the $[\mathrm{Ne} \mathrm{III}] 15.56 \mu \mathrm{m} /[\mathrm{Ne}$ II $] 12.81 \mu \mathrm{m}$ ratio from our spatially resolved measurements. As can be seen 

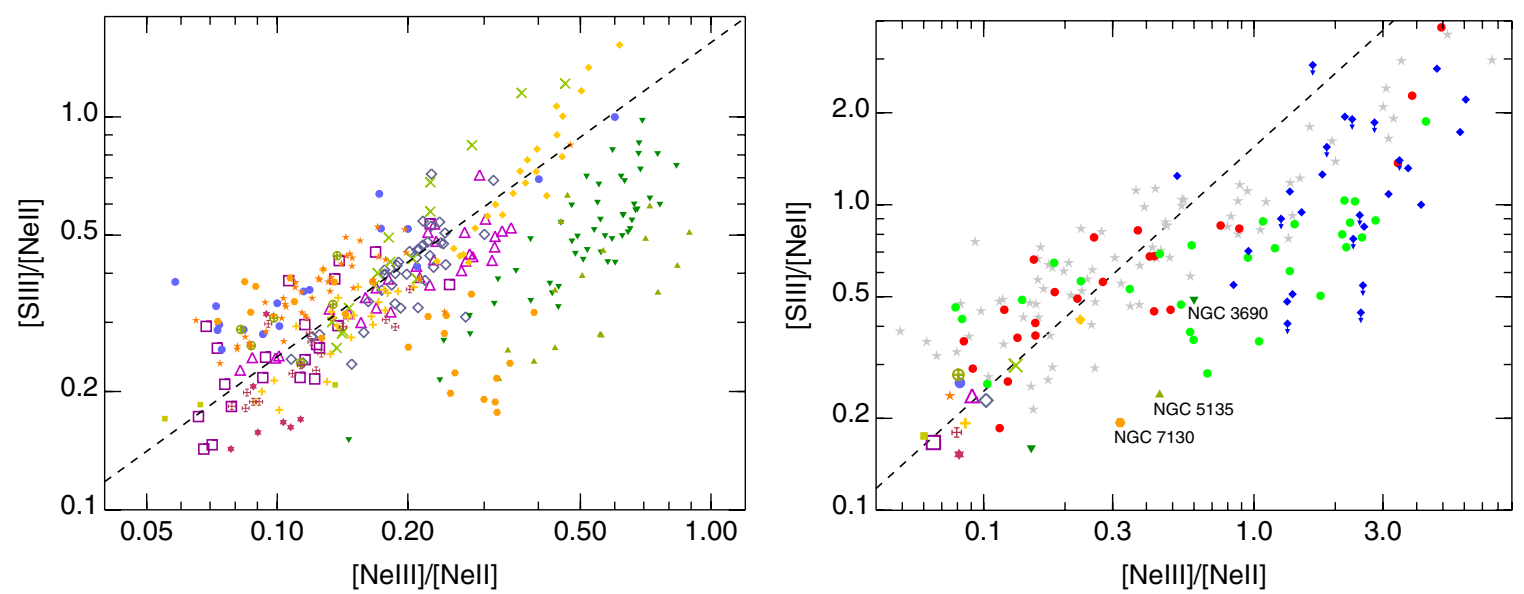

Figure 16. Left panel: [S III] $18.71 \mu \mathrm{m} /[\mathrm{Ne}$ II] $12.81 \mu \mathrm{m}$ ratio vs. [Ne III] $15.56 \mu \mathrm{m} /[\mathrm{Ne}$ II] $12.81 \mu \mathrm{m}$ ratio from the spatially resolved maps (Each point corresponds to a resolution element including the nuclei.). Right panel: nuclear ratios of our sample of LIRGs. We can compare them with: H II regions (grey stars) and star-forming galaxies (red circles) of Dale et al. (2009), Seyfert galaxies of Tommasin et al. (2008; green circles), and quasars of Veilleux et al. (2009; blue diamonds). The LIRG symbols are as in Figure 14. The black dashed line is the best fit to the spatially resolved data of the LIRGs excluding those galaxies containing an active nucleus which lie below the correlation. We did not apply an extinction correction.

(A color version of this figure is available in the online journal.)

from this figure, there is a tight correlation between the two line ratios if we exclude those galaxies hosting an active nucleus (NGC 3690, NGC 5135, and NGC 7130). The best linear fit to the data (excluding the AGNs) is

$$
\log (y)=0.19 \pm 0.02+(0.81 \pm 0.03) \times \log (x)
$$

where $y$ is the $[\mathrm{S} \mathrm{III]} 18.71 \mu \mathrm{m} /[\mathrm{Ne} \mathrm{II}] 12.81 \mu \mathrm{m}$ ratio and $x$ the $[\mathrm{Ne}$ III] $15.56 \mu \mathrm{m} /[\mathrm{Ne}$ II] $12.81 \mu \mathrm{m}$ ratio. In the right panel of Figure 16 we plot the nuclear ratios of our LIRGs together with those of extranuclear $\mathrm{H}$ II regions and nuclei of nearby galaxies (Dale et al. 2009), Seyfert galaxies (Tommasin et al. 2008), and quasars (Veilleux et al. 2009). Both diagrams show that, in general, for a given density (as traced by the [S III] $18.71 \mu \mathrm{m} /$ [Ne II] $12.81 \mu \mathrm{m}$ ratio) the AGNs show larger [Ne III] $15.56 \mu \mathrm{m} /$ [Ne II] $12.81 \mu \mathrm{m}$ ratios than starburst galaxies and $\mathrm{H}$ II regions. The most straightforward explanation is that the ionizing photons from the active nucleus increase the radiation hardness (the $[\mathrm{Ne}$ III $] 15.56 \mu \mathrm{m} /[\mathrm{Ne}$ II $] 12.81 \mu \mathrm{m}$ ratio). However some of the Seyfert galaxies and quasars are in the diagram close to $\mathrm{H}$ II regions. These may be galaxies harboring an AGN as well as star formation. Moreover the position of the AGNs and starbursts in Figure 16 is consistent with models (Groves et al. 2004; Dopita et al. 2006).

\subsubsection{The $[S$ IV] $10.51 \mu \mathrm{m}$ to [Ne III] $15.56 \mu \mathrm{m}$ and to [S III] $18.71 \mu \mathrm{m}$ Line Ratios}

The [S IV] $10.51 \mu \mathrm{m}$ and [Ne III] $15.56 \mu \mathrm{m}$ lines arise from ions with a similar excitation potential, $35 \mathrm{eV}$ and $41 \mathrm{eV}$, respectively. While the [Ne III] $15.56 \mu \mathrm{m}$ line is detected in all galaxies, the [S IV] $10.51 \mu \mathrm{m}$ line is detected in just six galaxies ( $40 \%$ of the sample). If the [S IV] $10.51 \mu \mathrm{m}$ emission arises from silicate dust embedded regions, it can be heavily affected by extinction as it is inside the broad $9.7 \mu \mathrm{m}$ silicate feature. This seems to be the case in ULIRGs (Farrah et al. 2007). However, the depths of the silicate features in our sample of LIRGs are moderate, and generally much lower than in ULIRGs probably indicating lower extinction (see Section 4).

Using the photoionization models of Snijders et al. (2007), we can infer the expected range of [S IV] $10.51 \mu \mathrm{m} /$ [Ne III] $15.56 \mu \mathrm{m}$ ratios. The values for the range of densities,

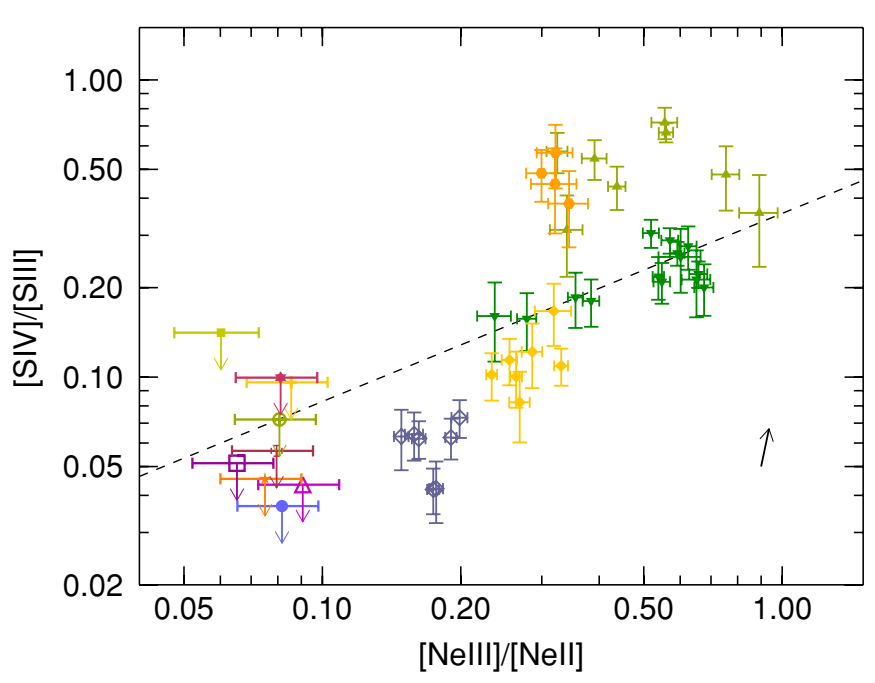

Figure 17. [S IV $] 10.51 \mu \mathrm{m} /[\mathrm{S}$ III] $18.71 \mu \mathrm{m}$ ratio vs. [Ne III] $15.56 \mu \mathrm{m} /$ $[\mathrm{Ne}$ II $] 12.81 \mu \mathrm{m}$ ratio from the spatially resolved maps. We did not apply an extinction correction. The galaxy symbols are as in Figure 14. When [S IV] $10.51 \mu \mathrm{m}$ is not detected we represent the upper limit of the nuclear [S IV $] 10.51 \mu \mathrm{m} /\left[\mathrm{S}_{\mathrm{III}}\right] 18.71 \mu \mathrm{m}$ ratio. The dashed line is the correlation found by Gordon et al. (2008) for M101 H II regions and starburst galaxies. The black arrow indicates the extinction correction of $\mathrm{A}_{V}=10 \mathrm{mag}$, assuming a screen geometry. The orange and light green points above the correlation corresponds to galaxies harboring an AGN (see Section 5.2.3).

(A color version of this figure is available in the online journal.)

ionization parameters, and stellar population ages covered by the models, excluding the most extreme cases, are compatible with the upper limits we measure. Moreover, we do not find any correlation between the $[\mathrm{S} \mathrm{IV}] 10.51 \mu \mathrm{m} /[\mathrm{Ne}$ III] $15.56 \mu \mathrm{m}$ ratio and the observed silicate strength $\left(S_{\mathrm{Si}}\right)$. Thus it is likely that the non-detection of the [S IV] $10.51 \mu \mathrm{m}$ line is because its flux is under the detection limit.

The $[\mathrm{S} \mathrm{IV}] 10.51 \mu \mathrm{m} /[\mathrm{S}$ III $] 18.71 \mu \mathrm{m}$ and the [Ne III] $15.56 \mu \mathrm{m} /[\mathrm{Ne}$ II $] 12.81 \mu \mathrm{m}$ ratios are well correlated and can be used to estimate the hardness of the radiation field (Verma et al. 2003; Dale et al. 2006; Gordon et al. 2008). Figure 17 shows that there is a good correlation between the [S IV] $10.51 \mu \mathrm{m} /$ [S III] $18.71 \mu \mathrm{m}$ and [Ne III] $15.56 \mu \mathrm{m} /[\mathrm{Ne}$ II] $12.81 \mu \mathrm{m}$ ratios 
of the spatially resolved measurements for our star-forming LIRGs, and indeed, they approximately follow the correlation found by Gordon et al. (2008) for M101 HiI regions and starburst galaxies. The upper limits to the [S IV] $10.51 \mu \mathrm{m} /$ [S III] $18.71 \mu \mathrm{m}$ ratio are also consistent with this trend.

Two of the galaxies classified as active, NGC 5135 and NGC 7130, lie above the Gordon et al. (2008) correlation. A similar trend was found by Dale et al. (2006) for AGN in the $\left[\mathrm{S}_{\mathrm{IV}}\right] 10.51 \mu \mathrm{m} /[\mathrm{S} \mathrm{III}] 33.48 \mu \mathrm{m}$ versus the $[\mathrm{Ne} \mathrm{III}] 15.56 \mu \mathrm{m} /$ $[\mathrm{Ne}$ II $] 12.81 \mu \mathrm{m}$ diagram. A possible explanation is that extra [S IV] $10.51 \mu \mathrm{m}$ emission is produced by the active nucleus. High spatial resolution ground-based observations of these two LIRGs indicate that most of the [S IV] $10.51 \mu \mathrm{m}$ emission comes from the nucleus, whereas this line is not detected in the surrounding star-forming regions of these galaxies (Díaz-Santos et al. 2010). For the other two galaxies NGC 3690 (classified as Seyfert), and NGC 7591 (classified as composite, intermediate between $\mathrm{H}_{\mathrm{II}}$ and LINER) the [S IV] $10.51 \mu \mathrm{m}$ line is more likely to be affected by extinction, as both have nuclear $S_{\mathrm{Si}} \sim-0.8$, and thus the observed [S IV] $10.51 \mu \mathrm{m} /[\mathrm{S}$ III $] 18.71 \mu \mathrm{m}$ ratios are lower limits.

\section{PAH FEATURES}

Models predict that the relative strength of the PAH bands depends on the ionization state (Draine \& Li 2001; Galliano 2006). The $6.2 \mu \mathrm{m}$ PAH and $7.7 \mu \mathrm{m}$ PAH bands dominate the emission of ionized PAH, whereas the $11.3 \mu \mathrm{m}$ PAH is more intense for neutral PAHs. More complete models of PAH feature behavior have been published recently by Galliano et al. (2008), who explore the effects of the radiation field hardness and intensity as well as the size distribution of the PAH carriers.

\subsection{Morphology}

Figure 1 shows flux maps of three brightest PAH features present in the SL module at $6.2 \mu \mathrm{m}, 7.7 \mu \mathrm{m}$, and $11.3 \mu \mathrm{m}$, together with the $5.5 \mu \mathrm{m}$ continuum maps. Table 6 shows the ratio between the flux arising from the central $2 \mathrm{kpc}$ and the total emission. The $11.3 \mu \mathrm{m}$ PAH is clearly more extended than the $5.5 \mu \mathrm{m}$ continuum in $60 \%$ of the galaxies, arising also from more diffuse regions (see also Díaz-Santos et al. 2010). However, the nuclear contribution to the integrated $6.2 \mu \mathrm{m}$ PAH emission is comparable to that at the $5.5 \mu \mathrm{m}$ continuum, and only in two cases (NGC 7130 and NGC 7591) the $6.2 \mu \mathrm{m}$ PAH emission is more extended than the continuum. When comparing both PAHs, the $11.3 \mu \mathrm{m}$ PAH emission is more extended than the $6.2 \mu \mathrm{m}$ PAH emission in about $40 \%$ of the galaxies, while the opposite is never the case.

A direct comparison of the $\mathrm{PAH}$ ratios with fine structure line ratios is not possible because the low spectral resolution $(R \sim 60-120)$ is not sufficient to separate the emission lines from the PAHs. Alternatively we used high-resolution $(R \sim 600)$ data to measure the fine structure lines and the $11.3 \mu \mathrm{m}$ PAH (Figure 4).

In general, the $11.3 \mu \mathrm{m}$ PAH emission appears to be more extended than the $[\mathrm{Ne}$ II] $12.81 \mu \mathrm{m}$ line. The PAH emission is also more extended than the $[\mathrm{Ne}$ II $] 12.81 \mu \mathrm{m}+[\mathrm{Ne}$ III $] 15.56 \mu \mathrm{m}$ emission which, as discussed in Section 5, traces recent star formation. PAHs have also been used to measure the star formation, since they are a proxy, although not perfect, for the total infrared luminosity (Peeters et al. 2004; Smith et al. 2007b). From this comparison, it is clear that the $11.3 \mu \mathrm{m}$ PAH emission does not trace the same stars as the [Ne II]12.81 $\mu \mathrm{m}$ emission line. In fact, Peeters et al. (2004) found that the $6.2 \mu \mathrm{m}$ PAH is not a good tracer of massive star formation (O stars) as seems to be the case of the $11.3 \mu \mathrm{m}$ PAH.

\subsection{Extinction Effects on the PAH Ratios}

As discussed above, models (Draine \& Li 2001; Galliano 2006) predict that neutral PAHs show larger $11.3 \mu \mathrm{m}$ PAH to $6.2 \mu \mathrm{m}$ PAH and $11.3 \mu \mathrm{m}$ PAH to $7.7 \mu \mathrm{m}$ PAH ratios than ionized PAHs. Figures 2 and 3 show the SL spectral maps of the $11.3 \mu \mathrm{m} \mathrm{PAH} / 6.2 \mu \mathrm{m}$ PAH and $11.3 \mu \mathrm{m} \mathrm{PAH} / 7.7 \mu \mathrm{m}$ PAH ratios, respectively. The $11.3 \mu \mathrm{m} \mathrm{PAH} / 6.2 \mu \mathrm{m}$ PAH ratio spans a factor of 2 , from $\sim 0.45$ to $\sim 1.15$ and the $11.3 \mu \mathrm{m} \mathrm{PAH} / 7.7 \mu \mathrm{m}$ $\mathrm{PAH}$ ratio range is $\sim 0.3-0.9$. These ranges are similar to those found in starburst galaxies (Brandl et al. 2006).

The $11.3 \mu \mathrm{m}$ PAH is affected by the broad $9.7 \mu \mathrm{m}$ silicate feature, thus in highly obscured regions the the $11.3 \mu \mathrm{m} \mathrm{PAH/}$ $6.2 \mu \mathrm{m}$ PAH and the $11.3 \mu \mathrm{m}$ PAH/7.7 $\mu \mathrm{m}$ PAH ratios can be underestimated. An example of this is the southern nucleus of NGC 3256, one of the most obscured regions $\left(S_{\mathrm{Si}}=-1.4\right)$ in the sample. Its $11.3 \mu \mathrm{m} \mathrm{PAH} / 6.2 \mu \mathrm{m}$ PAH ratio is lower than that in the surrounding regions.

To explore the effects of the $9.7 \mu \mathrm{m}$ silicate feature on the $\mathrm{PAH}$ ratios, we compare the $11.3 \mu \mathrm{m} \mathrm{PAH} / 6.2 \mu \mathrm{m} \mathrm{PAH}$ ratio and $S_{\mathrm{Si}}$ for the spatially resolved measurements of our LIRGs in Figure 18 (left panel). A similar figure was presented by Brandl et al. (2006) for the nuclear regions of starburst galaxies. They found that the extinction can change the relative strength of the PAHs by up to a factor of 2 . We find, however, that the median value of the $11.3 \mu \mathrm{m} \mathrm{PAH} / 6.2 \mu \mathrm{m}$ PAH ratio is approximately constant for silicate strengths weaker than $\sim-1.0$, and only deeper silicate absorptions seem to affect the $11.3 \mu \mathrm{m} \mathrm{PAH} /$ $6.2 \mu \mathrm{m}$ PAH ratio. Some caution is needed here because there are very few regions in our sample with silicate strengths stronger than -1.0 .

As an alternative approach, we corrected these $\mathrm{PAH}$ ratios for extinction using the spatially resolved measurements of the $S_{\mathrm{Si}}$. We assumed a foreground screen dust geometry and the extinction law of Smith et al. (2007b). For a typical value of the silicate strength in our sample of $S_{\mathrm{Si}}=-0.5$, this extinction reduces the $11.3 \mu \mathrm{m}$ PAH to $6.2 \mu \mathrm{m}$ PAH and to $7.7 \mu \mathrm{m}$ PAH ratios by $\sim 15 \%$. If we increase the silicate strength up to -1.0 the reduction is $\sim 30 \%$. These are much less than the factor of $\sim 2$ variations in the $11.3 \mu \mathrm{m} \mathrm{PAH} / 6.2 \mu \mathrm{m}$ PAH ratio observed in some galaxies (e.g., NGC 3256, NGC 3110, NGC 6701), at an almost constant value of $S_{\mathrm{Si}}$. Thus, we conclude that the observed variations in the PAH ratios are real, and do not significantly depend on the extinction, at least for regions in our sample of LIRGs with relatively shallow silicate absorptions $\left(S_{\mathrm{Si}}>-1\right)$.

\subsection{Nuclear versus Integrated PAH Ratios}

The right panel of Figure 18 compares the $11.3 \mu \mathrm{m} \mathrm{PAH} /$ $6.2 \mu \mathrm{m}$ PAH ratio versus $S_{\mathrm{Si}}$ values of the nuclear spectra and the integrated spectra. The general trend is that the integrated spectra have shallower silicate absorptions $\left(\sim 15 \%\right.$ lower $S_{\mathrm{Si}}$, on average) and slightly larger ( $20 \%$, on average) $11.3 \mu \mathrm{m}$ $\mathrm{PAH} / 6.2 \mu \mathrm{m}$ PAH ratios.

In Figure 19, we represent the $11.3 \mu \mathrm{m} \mathrm{PAH} / 6.2 \mu \mathrm{m}$ PAH ratio versus $7.7 \mu \mathrm{m} \mathrm{PAH} / 6.2 \mu \mathrm{m} \mathrm{PAH}$ ratio, comparing the nuclear and the integrated values of each galaxy. The distribution of the values of the ratio is approximately uniform. However it is interesting to note two clearly different behaviors. The majority 

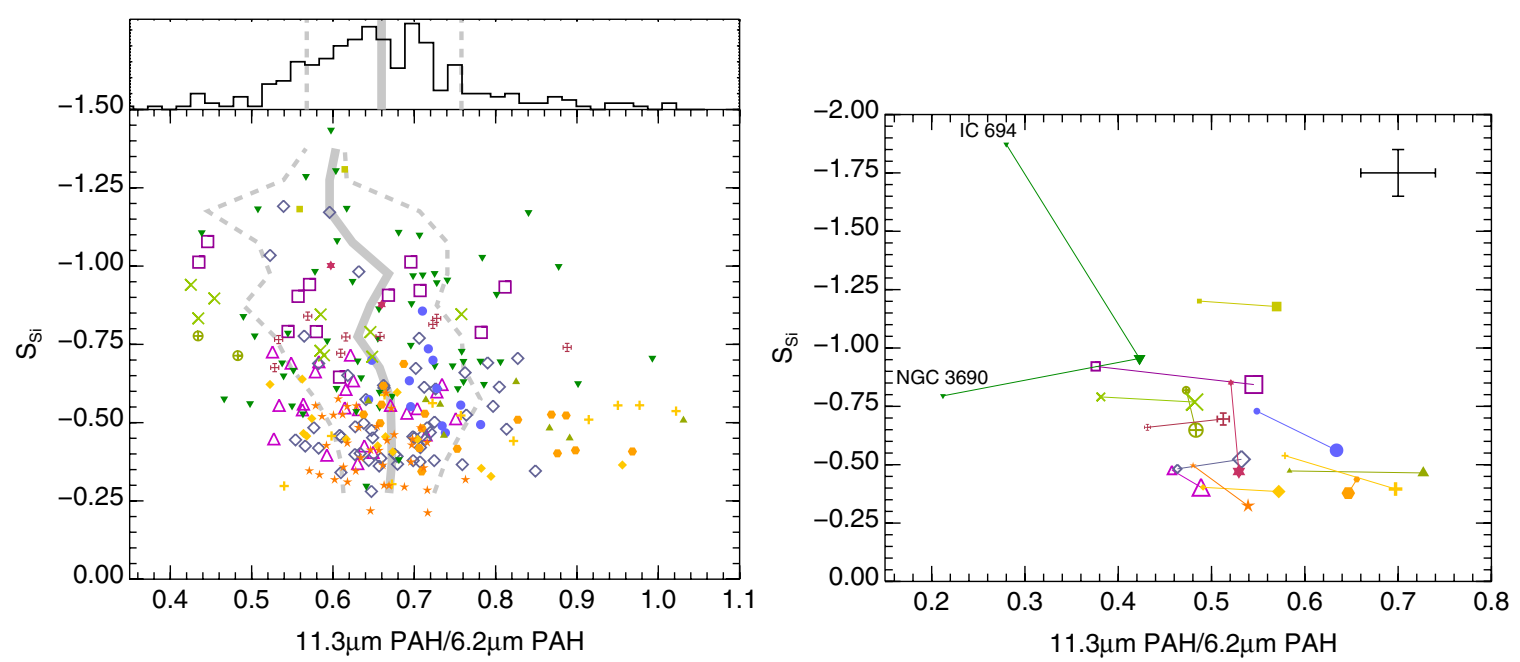

Figure 18. $11.3 \mu \mathrm{m} \mathrm{PAH} / 6.2 \mu \mathrm{m}$ PAH ratio vs. silicate strength $\left(S_{\mathrm{Si}}\right)$. Left: spatially resolved measurements using a $2 \times 2$ pixel box. The symbols are as in Figure 14 . The solid gray line is the median and the dashed gray lines encircle the region containing $68 \%$ of the data points. These are calculated in intervals of 0.25 of the $S_{\mathrm{Si}}$. The top histogram is the distribution of the the $11.3 \mu \mathrm{m} \mathrm{PAH} / 6.2 \mu \mathrm{m}$ PAH ratio for the whole sample of LIRGs, in gray are marked the median and the $1 \sigma$. Right: same as the left panel, but comparing the nuclear (small symbols) and integrated (large symbols) values. For each galaxy the values are connected by a line to guide the eye.

(A color version of this figure is available in the online journal.)

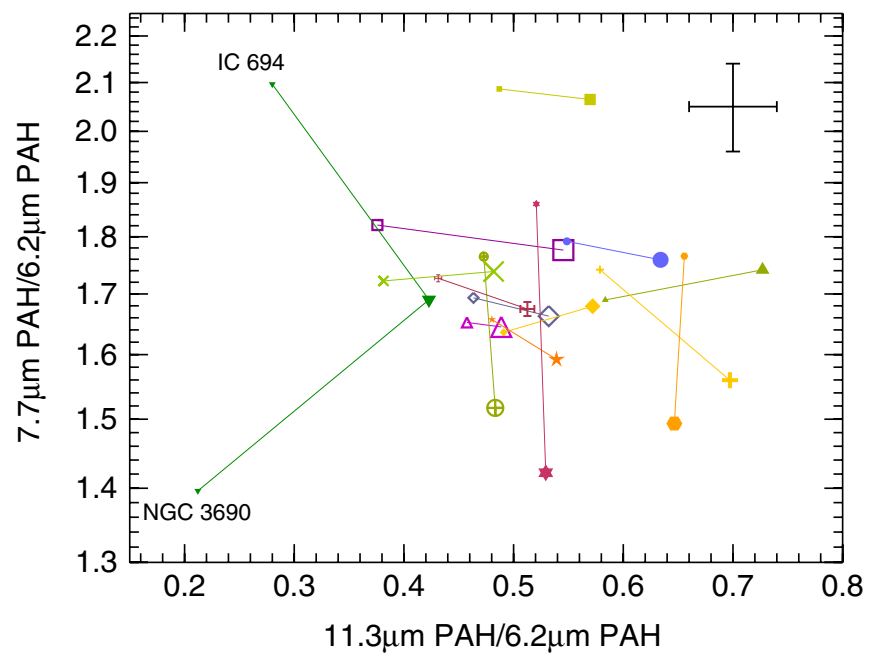

Figure 19. $11.3 \mu \mathrm{m} \mathrm{PAH} / 6.2 \mu \mathrm{m}$ PAH ratio vs. $7.7 \mu \mathrm{m} \mathrm{PAH} / 6.2 \mu \mathrm{m}$ PAH ratio for nuclear and integrated values. Symbols are as in the right panel of Figure 18. (A color version of this figure is available in the online journal.)

of the galaxies show a small variation of the $7.7 \mu \mathrm{m} \mathrm{PAH} /$ $6.2 \mu \mathrm{m}$ PAH ratio and a somewhat larger $11.3 \mu \mathrm{m} \mathrm{PAH} / 6.2 \mu \mathrm{m}$ $\mathrm{PAH}$ ratio in the integrated spectrum. This behavior is expected, since the $7.7 \mu \mathrm{m}$ PAH and $6.2 \mu \mathrm{m}$ PAH are related to ionized PAHs and the $11.3 \mu \mathrm{m}$ PAH to neutral PAHs. The ionized PAHs emission is concentrated in the nuclear regions, but there is a substantial amount of the $11.3 \mu \mathrm{m}$ PAH emission arising from diffuse regions throughout the galaxies (see Section 6.1). This explains the larger integrated $11.3 \mu \mathrm{m}$ PAH/6.2 $\mu \mathrm{m}$ PAH ratios of all the LIRGs (see Table 3).

Some galaxies, however, show a completely different behavior, with an almost constant $11.3 \mu \mathrm{m} \mathrm{PAH} / 6.2 \mu \mathrm{m}$ PAH ratio but a lower $7.7 \mu \mathrm{m} \mathrm{PAH} / 6.2 \mu \mathrm{m}$ PAH ratio in the integrated spectrum. The most extreme cases of this behavior are NGC 7130 and NGC 7591. Galliano et al. (2008) showed that the $7.7 \mu \mathrm{m}$ $\mathrm{PAH} / 6.2 \mu \mathrm{m}$ PAH ratio is very sensitive to the size distribution of the PAHs, with smaller PAH molecules producing lower $7.7 \mu \mathrm{m} \mathrm{PAH} / 6.2 \mu \mathrm{m}$ PAH ratios. One possibility is that smaller
PAH molecules are destroyed in harsher environments, such as those of Seyfert and LINER nuclei. The destruction of small PAHs might suppress the nuclear $6.2 \mu \mathrm{m}$ PAH emission. This may lead to the different behavior observed in the AGN/LINER nuclei in Figure 19. Moreover, the absolute value of the ratio seems to give little information about the nature, starburst or AGN, of the nuclei and we can only distinguish them by the relative variation between nuclear and integrated ratios.

\subsection{The Effects of the Radiation Field}

Since the $[\mathrm{Ne}$ III $] 15.56 \mu \mathrm{m} /[\mathrm{Ne}$ II] $12.81 \mu \mathrm{m}$ ratio is a proxy for the hardness of the radiation field, we can compare the spatial variations of this ratio with those of the $11.3 \mu \mathrm{m} \mathrm{PAH} / 6.2 \mu \mathrm{m}$ $\mathrm{PAH}$ to ascertain if the radiation field has an effect on the PAH ratios. A comparison between Figures 2 and 5 indicates that the $11.3 \mu \mathrm{m} \mathrm{PAH} / 6.2 \mu \mathrm{m}$ PAH ratio does not decrease necessarily in regions of enhanced [Ne III] $15.56 \mu \mathrm{m} /[\mathrm{Ne}$ II] $12.81 \mu \mathrm{m}$ ratios.

On the other hand, the morphology of the $11.3 \mu \mathrm{m}$ $\mathrm{PAH} / 7.7 \mu \mathrm{m}$ PAH ratio (Figure 3) resembles that of the $[\mathrm{Ne}$ III] $15.56 \mu \mathrm{m} /[\mathrm{Ne}$ II] $12.81 \mu \mathrm{m}$ ratio. However this resemblance is the opposite of what one would expect. Both ratios present their minima in the nuclear regions for most of the galaxies. Thus while the observed nuclear [Ne III] $15.56 \mu \mathrm{m} /$ [Ne II] $12.81 \mu \mathrm{m}$ ratio would indicate lower radiation hardness in the nuclei, the PAH ratio would suggest a larger ionization state. Again, it is likely that the typical radiation field hardness observed in our sample of LIRGs is not sufficiently large as to affect the PAH emission, as is the case of the low-metallicity galaxies of Wu et al. (2006). Another possible interpretation is that the PAHs and the neon emission are related to different physical processes that due to our limited spatial resolution we cannot separate. As a result, the bulk of the $7.7 \mu \mathrm{m}$ PAH emission may be produced in star-forming regions, while the $11.3 \mu \mathrm{m}$ $\mathrm{PAH}$ emission also comes from diffuse regions. Thus, low values of the $11.3 \mu \mathrm{m}$ PAH/7.7 $\mu \mathrm{m}$ PAH ratio trace H II regions, and larger values indicate less recent star formation. The latter interpretation is similar to that of Galliano et al. (2008). They find that the lowest values of the $11.3 \mu \mathrm{m} \mathrm{PAH} / 7.7 \mu \mathrm{m} \mathrm{PAH}$ 


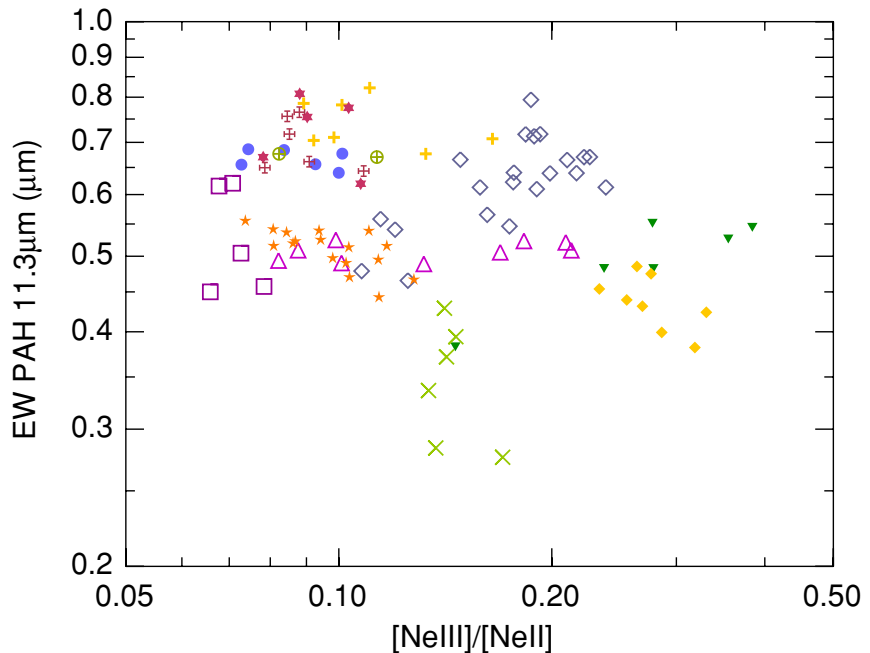

Figure 20. Spatially resolved measurements of the EW of the $11.3 \mu \mathrm{m}$ PAH vs. the $[\mathrm{Ne} \mathrm{III}] 15.56 \mu \mathrm{m} /[\mathrm{Ne} \mathrm{II}] 12.81 \mu \mathrm{m}$ ratio for those galaxies not classified as active. Galaxy symbols as in Figure 14.

(A color version of this figure is available in the online journal.)

ratio are associated with the brightest PAH emission. However, in their galaxies, the $11.3 \mu \mathrm{m} \mathrm{PAH} / 6.2 \mu \mathrm{m}$ PAH ratio follows the same trend, whereas this is not the case for the LIRGs studied here. Alternatively, as discussed in the previous section, the size of the grains may affect this ratio. If the smaller grains are favored in and around the nucleus, the $7.7 \mu \mathrm{m}$ PAH emission would be increased with respect to the $11.3 \mu \mathrm{m}$ PAH emission. As a consequence, the $11.3 \mu \mathrm{m} \mathrm{PAH} / 7.7 \mu \mathrm{m}$ PAH ratio would be lower in the nucleus.

Brandl et al. (2006) studied the dependency of the EW of the PAHs on the radiation hardness for a sample of highmetallicity starburst galaxies. Wu et al. (2006) carried out a similar study, but for low-metallicity galaxies. Whereas Brandl et al. (2006) found that this dependency does not exist, $\mathrm{Wu}$ et al. (2006) found the opposite, that is, the harsher radiation of low-metallicity galaxies field lowers the PAH EW. Eventually Gordon et al. (2008) showed that the reason for the discrepancy is the different range of the radiation field hardness in highand low-metallicity galaxies. In particular, Gordon et al. (2008) found that for ionizations above a certain threshold there is a correlation between the radiation hardness and the PAH EW, but for lower ionizations this dependency disappears. We show in Figure 20 the EW of the $11.3 \mu \mathrm{m}$ PAH and the [Ne III] $15.56 \mu \mathrm{m} /$ $[\mathrm{Ne}$ II $] 12.81 \mu \mathrm{m}$ ratio of those galaxies not classified as AGN. The ionization and metallicity of our LIRGs are similar to those galaxies studied by Brandl et al. (2006) and, similarly to their findings, there is no correlation between these parameters in our sample of LIRGs.

\subsection{Dependency on the Age of the Stellar Population}

Since the $[\mathrm{Ne}$ II $] 12.81 \mu \mathrm{m}$ emission traces young stellar populations $(<10 \mathrm{Myr})$, and since the $11.3 \mu \mathrm{m}$ PAH feature can also be excited by older populations (see Peeters et al. 2004 and Section 6.1), the [Ne II] $12.81 \mu \mathrm{m} / 11.3 \mu \mathrm{m}$ PAH ratio can give us an indication of age variations throughout the galaxies (Díaz-Santos et al. 2010). As can be seen from Figure 5, the $[\mathrm{Ne}$ II $] 12.81 \mu \mathrm{m} / 11.3 \mu \mathrm{m}$ PAH ratio ranges from $\sim 0.1$ to $\sim 0.6$ for our sample of LIRGs. In some LIRGs (e.g., NGC 6701, NGC 7130, NGC 7771, IC 4687), the [Ne II]12.81 $\mu \mathrm{m} / 11.3 \mu \mathrm{m}$ $\mathrm{PAH}$ ratio appears enhanced in the high surface brightness $\mathrm{H}$ II
Table 7

Molecular Hydrogen Emission Line Fluxes

\begin{tabular}{lcccc}
\hline \hline \multicolumn{1}{c}{ Name } & Size $^{\mathrm{a}}$ & $\begin{array}{c}\mathrm{H}_{2} \mathrm{~S}(2) \\
12.27 \mu \mathrm{m}\end{array}$ & $\begin{array}{c}\mathrm{H}_{2} \mathrm{~S}(1) \\
17.03 \mu \mathrm{m}\end{array}$ & $\begin{array}{c}\mathrm{H}_{2} \mathrm{~S}(0) \\
28.22 \mu \mathrm{m}\end{array}$ \\
\hline NGC 2369 & 2.9 & $10 . \pm 1$. & $19 . \pm 2$. & $3.5 \pm 0.5$ \\
NGC 3110 & 4.6 & $4 . \pm 1$. & $10 . \pm 0.9$ & $1.6 \pm 0.2$ \\
NGC 3256 & 2.7 & $34 . \pm 3$. & $61 . \pm 5$. & $9 . \pm 3$. \\
NGC 3690 & 2.9 & $11 . \pm 4$. & $22 . \pm 1$. & $3.8 \pm 0.6$ \\
IC 694 & 2.9 & $20 . \pm 3$. & $29 . \pm 1$. & $5.7 \pm 0.9$ \\
ESO 320-G030 & 2.9 & $8.1 \pm 1.2$ & $13 . \pm 1$. & $1.7 \pm 0.7$ \\
NGC 5135 & 3.9 & $9.3 \pm 1.4$ & $17 . \pm 1$. & $1.3 \pm 0.5$ \\
IC 4518E & 4.4 & $0.3 \pm 0.1$ & $1.1 \pm 0.2$ & $1.0 \pm 0.1$ \\
Zw 049.057 & 3.7 & $3.3 \pm 0.8$ & $5.8 \pm 0.4$ & $<0.6$ \\
IRAS 17138-1017 & 4.9 & $6.3 \pm 1.3$ & $14 . \pm 1$. & $1.9 \pm 0.5$ \\
IC 4687 & 4.9 & $6.5 \pm 1.1$ & $12 . \pm 1$. & $1.8 \pm 0.4$ \\
NGC 6701 & 3.7 & $4.6 \pm 0.8$ & $10 . \pm 1$. & $1.9 \pm 0.9$ \\
NGC 7130 & 4.6 & $4.2 \pm 1.7$ & $9.9 \pm 0.7$ & $1.4 \pm 0.3$ \\
IC 5179 & 3.2 & $6.3 \pm 0.8$ & $11.2 \pm 0.8$ & $1.4 \pm 0.2$ \\
NGC 7591 & 4.6 & $3.0 \pm 1.0$ & $5.5 \pm 0.6$ & $1.0 \pm 0.3$ \\
NGC 7771 & 4.1 & $8.7 \pm 1.2$ & $16 . \pm 1$. & $3.5 \pm 1.8$ \\
\hline
\end{tabular}

Notes. Fluxes are expressed in units of $10^{-17} \mathrm{~W} \mathrm{~m}^{-2}$.

a Projected square size of the aperture in kpc. The aperture size is $13^{\prime \prime} .4 \times 13^{\prime \prime}$. 4 for all the galaxies.

regions (see the $H S T$ /NICMOS Pa $\alpha$ images in Figure 4) with respect to regions of more diffuse emission. In other galaxies the interpretation might not be as straightforward. In some cases the IRS angular resolution is not sufficiently high to isolate the circumnuclear H II regions (e.g., NGC 5135), and the age differences may be smoothed out.

\section{MOLECULAR HYDROGEN}

We constructed maps of the molecular hydrogen emission using the pure rotational lines $\mathrm{H}_{2} \mathrm{~S}(1)$ at $17.0 \mu \mathrm{m}$ and the $\mathrm{H}_{2}$ $\mathrm{S}(2)$ at $12.3 \mu \mathrm{m}$ (Figure 4). These lines trace warm $(300 \mathrm{~K}$ $<T<1000 \mathrm{~K})$ molecular gas in the interstellar medium. The $\mathrm{H}_{2} \mathrm{~S}(1)$ morphology appears, generally, different from that of the fine structure lines, which are associated with the ionized gas medium. There are also differences in the velocity fields that will be discussed in Section 8. Using the same procedure as described in Section 5.1 we find that the $\mathrm{H}_{2} \mathrm{~S}(1)$ emission is more extended than the $15 \mu \mathrm{m}$ continuum in $\sim 60 \%$ of the sample. In most of these galaxies the size of the $\mathrm{H}_{2} \mathrm{~S}(1)$ emitting region is larger than that of the fine structure lines (see Table 6).

\subsection{Temperatures, Column Densities, and Masses}

We extracted the nuclear spectra of all galaxies using a 13". $4 \times$ 13."4 aperture. This aperture corresponds to physical scales between 3 and $5 \mathrm{kpc}$ depending on the galaxy. This aperture size is different from that used in the other sections because now we are limited by the lowest spatial resolution of the $\mathrm{LH}$ module, where the $\mathrm{H}_{2} \mathrm{~S}(0)$ line at $28.2 \mu \mathrm{m}$ lies. The fluxes of the $\mathrm{H}_{2}$ lines measured from these spectra are listed in Table 7.

The temperature of the warm molecular hydrogen was calculated as described by Roussel et al. (2007). We assumed an ortho-to-para ratio $=3$ (Rigopoulou et al. 2002; Roussel et al. 2007), and thus this assumption may introduce some uncertainty as it is used in the temperature calculations. The column densities and masses are highly dependent on the $\mathrm{H}_{2}$ lines used in these calculations. It is preferable to use the lines $\mathrm{H}_{2} \mathrm{~S}(0)$ and $\mathrm{H}_{2} \mathrm{~S}(1)$ because they come from the lowest rotational levels of the hydrogen molecule, and therefore they trace the molecular 
Table 8

Derived Warm Molecular Hydrogen Temperatures, Column Densities, and Masses

\begin{tabular}{|c|c|c|c|c|c|c|}
\hline Name & $\begin{array}{c}T(\mathrm{~S}(0)-\mathrm{S}(1)) \\
(\mathrm{K})\end{array}$ & $\begin{array}{c}N_{0} \\
\left(\times 10^{20} \mathrm{~cm}^{-2}\right) \\
\end{array}$ & $\begin{array}{c}M_{0} \\
\left(\times 10^{8} M_{\odot}\right)\end{array}$ & $\begin{array}{c}T(\mathrm{~S}(1)-\mathrm{S}(2)) \\
(\mathrm{K})\end{array}$ & $\begin{array}{c}N_{1} \\
\left(\times 10^{20} \mathrm{~cm}^{-2}\right) \\
\end{array}$ & $\begin{array}{c}\mathrm{M}_{1} \\
\left(\times 10^{8} M_{\odot}\right) \\
\end{array}$ \\
\hline NGC 2369 & 168 & 8.8 & 1.3 & 348 & 5.1 & 0.73 \\
\hline NGC 3110 & 178 & 3.7 & 1.3 & 305 & 2.4 & 0.86 \\
\hline NGC 3256 & 181 & 19. & 2.1 & 369 & 12. & 1.3 \\
\hline NGC 3690 & 172 & 8.9 & 1.2 & 351 & 5.3 & 0.72 \\
\hline IC 694 & 163 & 15. & 2.0 & 415 & 7.8 & 1.1 \\
\hline ESO 320-G030 & 189 & 3.4 & 0.43 & 390 & 2.2 & 0.28 \\
\hline NGC 5135 & 236 & 1.9 & 0.44 & 361 & 1.5 & 0.36 \\
\hline IC $4518 \mathrm{E}$ & 108 & 9.2 & 2.7 & 252 & 3.0 & 0.87 \\
\hline Zw 049.057 & $>205$ & $<1.0$ & $<0.22$ & 373 & 0.74 & 0.16 \\
\hline IRAS $17138-1017$ & 185 & 4.1 & 1.5 & 324 & 2.7 & 1.0 \\
\hline IC 4687 & 178 & 4.1 & 1.5 & 361 & 2.5 & 0.92 \\
\hline NGC 6701 & 168 & 4.7 & 1.0 & 323 & 2.8 & 0.61 \\
\hline NGC 7130 & 183 & 3.0 & 0.97 & 320 & 2.0 & 0.65 \\
\hline IC 5179 & 194 & 2.6 & 0.40 & 369 & 1.7 & 0.27 \\
\hline NGC 7591 & 167 & 2.5 & 0.85 & 362 & 1.4 & 0.48 \\
\hline NGC 7771 & 157 & 10.0 & 2.6 & 363 & 5.2 & 1.3 \\
\hline
\end{tabular}

Notes. $N_{0}$ and $M_{0}$ are the column density and mass derived from the $\mathrm{H}_{2}$ lines $\mathrm{S}(0)$ and $\mathrm{S}(1) . N_{1}$ and $M_{1}$ correspond to the column density and mass derived from the $\mathrm{S}(1)$ and $\mathrm{S}(2)$ lines.

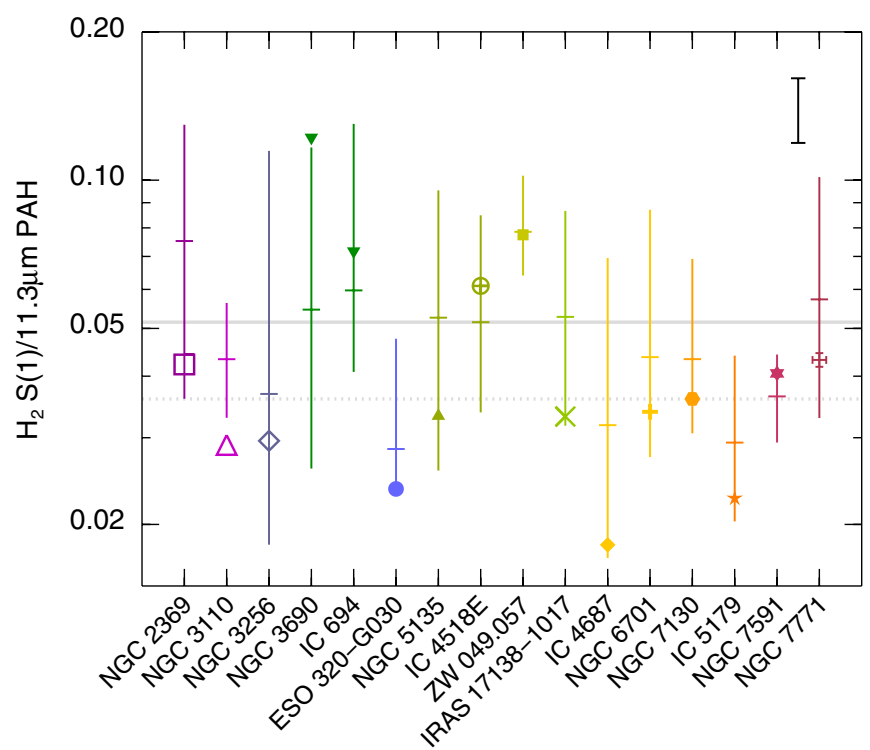

Figure 21. Range of the observed $\mathrm{H}_{2} \mathrm{~S}(1) / 11.3 \mu \mathrm{m}$ PAH ratio for each galaxy. Symbols are as in Figure 14.

(A color version of this figure is available in the online journal.)

hydrogen gas at the lowest temperatures which dominates determination of the masses (Roussel et al. 2007). However the $\mathrm{H}_{2}$ $\mathrm{S}(0)$ line is not detected in all galaxies, and to allow meaningful comparisons we estimated the column densities and masses with the $\mathrm{H}_{2} \mathrm{~S}(1)$ and $\mathrm{H}_{2} \mathrm{~S}(2)$ lines. The results are presented in Table 8. The masses estimated from the $\mathrm{H}_{2} \mathrm{~S}(1)$ and $\mathrm{H}_{2} \mathrm{~S}(0)$ lines are in the range $(0.4-3) \times 10^{8} M_{\odot}$. These values are comparable to those found in ULIRGs (Higdon et al. 2006), local starbursts, and Seyfert galaxies (Rigopoulou et al. 2002; Tommasin et al. 2008). It should be noted that these masses correspond to a beam size of 13 ". $4 \times 13$ ". 4 which does not include all the molecular hydrogen emission. Then the mass values listed in Table 8 should be considered as lower limits to the total $\mathrm{H}_{2}$ mass of the galaxy. For instance, the integrated fluxes of the $\mathrm{H}_{2} \mathrm{~S}(1)$ line (Table 4) are a factor of between 2 and 3 larger than those used in the mass calculations (Table 7). Thus assuming that the physical

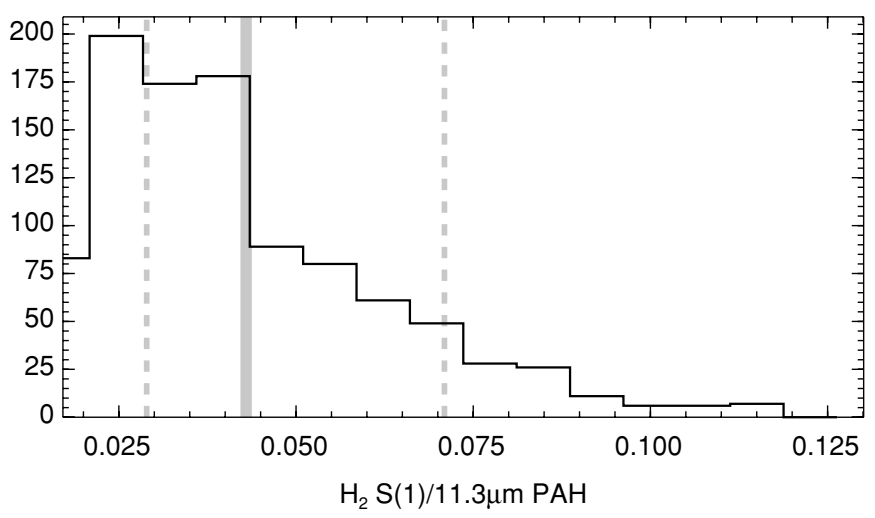

Figure 22. Distribution of the observed $\mathrm{H}_{2} \mathrm{~S}(1) / 11.3 \mu \mathrm{m}$ PAH ratio for all the galaxies from spatially resolved measurements. The lines are as in Figure 11.

conditions of the $\mathrm{H}_{2}$ in the outer parts of the galaxy are similar to those in the inner $\sim 3-5 \mathrm{kpc}$, the total mass would be at least a factor of $\sim 2-3$ larger than the values listed in Table 8 .

\subsection{Excitation Mechanism and Relation to the PAH Emission}

Studies of the molecular hydrogen emission of galactic PDRs indicate that the formation of $\mathrm{H}_{2}$ occurs in the surfaces of PAHs (see, e.g., Habart et al. 2003; Velusamy \& Langer 2008) as both the PAH carriers and molecular hydrogen can be excited by UV radiation. Thus the $\mathrm{H}_{2}$ and the $\mathrm{PAH}$ emission morphologies are correlated in these regions. A similar correlation is seen in external galaxies (Rigopoulou et al. 2002; Roussel et al. 2007). However, $\mathrm{H}_{2}$ can be excited by other mechanisms, such as X-rays (e.g., Lepp \& McCray 1983) and shock fronts (e.g., Hollenbach \& McKee 1989).

To explore the morphological differences we constructed maps of the $\mathrm{H}_{2} \mathrm{~S}(1) / 11.3 \mu \mathrm{m} \mathrm{PAH}$ ratio. The range of this ratio for each galaxy is shown in Figure 21. This ratio varies by a factor of 10 in our sample of LIRGs, although, as can be seen in the Figure 22, 68\% of the values are in a narrower range, from 0.028 to 0.073 . Figure 23 shows the ratio maps of those galaxies with the most noticeable morphological characteristics. 

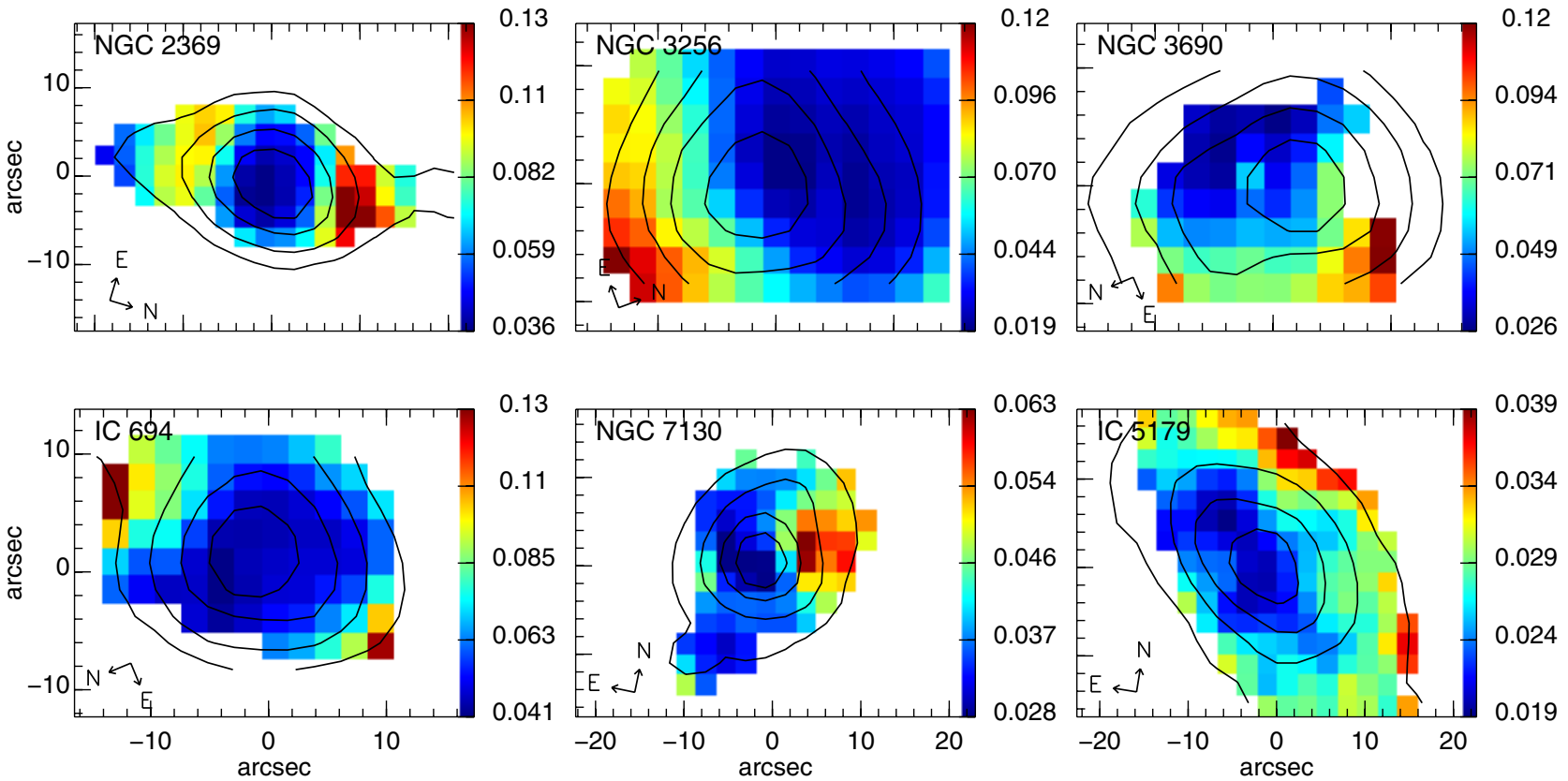

Figure 23. Maps of the observed $\mathrm{H}_{2} \mathrm{~S}(1) / 11.3 \mu \mathrm{m}$ PAH ratio for selected galaxies. For reference we represent the $15 \mu \mathrm{m}$ continuum contours.

(A color version of this figure is available in the online journal.)

The $11.3 \mu \mathrm{m}$ PAH feature falls close to the the $9.7 \mu \mathrm{m}$ silicate absorption and, although the $\mathrm{H}_{2} \mathrm{~S}(1)$ is also close to the silicate absorption at $18 \mu \mathrm{m}$, extinction variations could increase artificially the $\mathrm{H}_{2} \mathrm{~S}(1) / 11.3 \mu \mathrm{m}$ PAH ratio. We used the extinction curve adopted by Smith et al. (2007b) to quantify this effect. If we assume a mixed model for the dust distribution, the ratio can be increased by up to a factor of $\sim 1.4$, for the typical silicate absorption observed in our sample of LIRGs. Which is much lower than the observed variation. Choosing a screen geometry, to increase the ratio by a factor of 2 we would need an optical extinction of $A_{V} \sim 100 \mathrm{mag}$, which is much larger than our estimates (Section 4). Therefore, the effect of the extinction is small compared to the observed variations of the $\mathrm{H}_{2} \mathrm{~S}(1) / 11.3 \mu \mathrm{m}$ PAH ratio.

We can try to distinguish among the different excitation mechanisms by comparing the $11.3 \mu \mathrm{m}$ PAH morphology with that of the $\mathrm{H}_{2}$ emission (Figure 4). It is clear that the morphologies are different. The $\mathrm{H}_{2} \mathrm{~S}(1)$ emission is more extended than the PAH emission, although the maxima of both emissions are located in the nucleus for most of the galaxies. The only exception is NGC 3256, where the $\mathrm{H}_{2} \mathrm{~S}(1)$ peaks at the southern nucleus while the PAH maximum is located at northern nucleus. We note, however, that the southern nucleus of this galaxy is very obscured (see Figure 8 and Díaz-Santos et al. 2010), and the $11.3 \mu \mathrm{m}$ PAH map is not corrected for extinction. This suggests that the warm $\mathrm{H}_{2}$ in LIRGs is produced not only in PDRs, but that the other mechanisms also play a role.

We attribute the increased values of the $\mathrm{H}_{2} \mathrm{~S}(1) / 11.3 \mu \mathrm{m}$ $\mathrm{PAH}$ ratio, not to a deficiency of $\mathrm{PAH}$ emission, but rather to an excess emission by $\mathrm{H}_{2}$, excited by X-ray or shocks. For instance, in the merger system NGC 3256, the largest value of the ratio occurs where the components of the merger are colliding. The $\mathrm{H}_{2}$ line ratios are inconsistent with $\mathrm{X}$-ray excitation (Shaw et al. 2005). Thus, it is likely that there is some contribution from shocks to the $\mathrm{H}_{2}$, as appears also to be the case for some regions in NGC 3690 and IC 694 (Alonso-Herrero et al. 2009b). In many cases, the hypothesis of shock excitation is supported by large velocity dispersions (see García-Marín et al. 2006 for NGC 3690/IC 694 and the following section for additional examples).

The galaxy IC 5179 is interesting because its velocity field (see the next section) is highly ordered, so strong shocks are unlikely, and its X-ray luminosity is low. Thus the $\mathrm{H}_{2}$ emission in this galaxy is likely to be produced in PDRs. The small variations (a factor of 2) of the $\mathrm{H}_{2} \mathrm{~S}(1) / 11.3 \mu \mathrm{m}$ PAH ratio are probably due to differences in the extinction or differences in the $11.3 \mu \mathrm{m}$ PAH relative strength with respect to the total PAH luminosity from region to region. For the rest of the galaxies, the morphology of $\mathrm{H}_{2} \mathrm{~S}(1) / 11.3 \mu \mathrm{m}$ PAH ratio does not allow us to determine whether the $\mathrm{H}_{2}$ is produced in PDRs or is excited by shock fronts or X-ray.

\section{VELOCITY FIELDS}

Figure 10 shows the velocity fields of the [Ne II] $12.81 \mu \mathrm{m}$ and $\mathrm{H}_{2} \mathrm{~S}(1)$ emission lines in the $\mathrm{SH}$ module. They cover $\sim 30^{\prime \prime} \times 30^{\prime \prime}$, which corresponds to physical scales of $\sim 6-11 \mathrm{kpc}$, depending on the galaxy. Most of the $[\mathrm{Ne}$ II $] 12.81 \mu \mathrm{m}$ velocity fields are consistent with a rotating disk, although some of them show more complex morphologies (NGC 3110, NGC 3256, IC 694, NGC 6701, and NGC 7130). Only two of the galaxies (NGC 3256 and IC 694) with perturbed velocity fields are part of merger systems. NGC 3110 and NGC 7130 are paired with other galaxies. However not all the galaxies in groups present disturbed velocity fields (e.g., NGC 7771, IC $4518 \mathrm{E})$, at least on the physical scales $(\sim \mathrm{kpc})$ probed by the IRS spectral maps.

Figure 24 shows the velocity field of the interacting system Arp 299 (IC 694 and NGC 3690) rotated to the usual north up east to the left orientation for easy comparison with other works. The $[\mathrm{Ne}$ II $] 12.81 \mu \mathrm{m}$ velocity field, which traces the ionized gas, agrees well with that measured from $\mathrm{H} \alpha$ (see García-Marín et al. 2006). The peak-to-peak variation of the velocity field $\sim 250 \mathrm{~km} \mathrm{~s}^{-1}$ is also comparable to that measured from $\mathrm{H} \alpha$. 

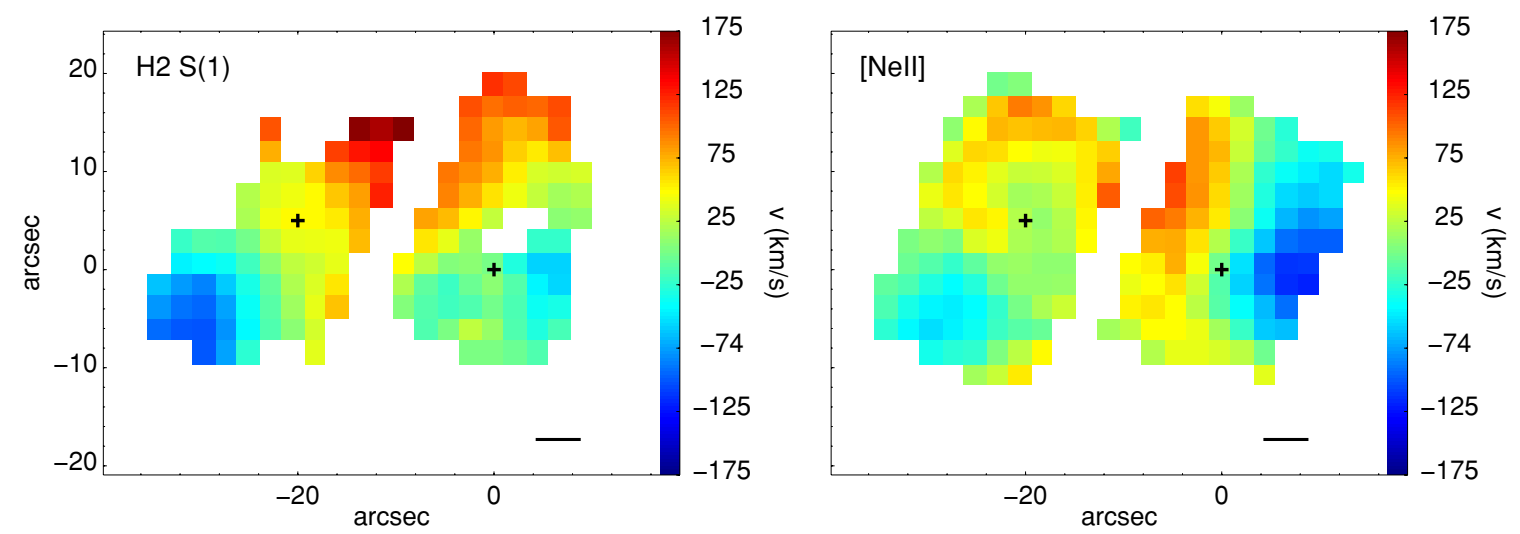

Figure 24. Arp 299 (NGC 3690 and IC 694) velocity fields of the molecular gas traced by the $\mathrm{H}_{2} \mathrm{~S}(1)$ line (left) and the ionized gas traced by the [Ne II] $12.81 \mu \mathrm{m}$ line (right). The velocities are referred to the the nucleus B1 of Arp 299. The black crosses mark the nuclei A and B1 of Arp 299. The scale represents 1 kpc. North is up and east to the left.

(A color version of this figure is available in the online journal.)

The warm molecular gas $(300 \mathrm{~K}<T<1000 \mathrm{~K}$ ) velocity field, traced by the $\mathrm{H}_{2} \mathrm{~S}(1)$ line, is similar to the $[\mathrm{Ne} \mathrm{II}] 12.81 \mu \mathrm{m}$ field (Figure 24), as both rotate in the same sense, although there are differences. The molecular hydrogen velocity field does not present the irregular structure of the ionized gas and the neutral gas (see García-Marín et al. 2006). However, the $\mathrm{H}_{2}$ $S(1)$ velocity field resembles that produced by two disks rotating in opposite sense. This suggests that before the interaction both galaxies were spirals (Augarde \& Lequeux 1985). Moreover the $\mathrm{H}_{2} \mathrm{~S}(1)$ velocity field shows evident similarities with the $\mathrm{CO}$ velocity field (see Aalto et al. 1997; Casoli et al. 1999).

$\mathrm{H} \alpha$ velocity fields are available for NGC 3256 (Lípari et al. 2000), NGC 6701 and NGC 7771 (Alonso-Herrero et al. 2009a), and NGC 7591 (Amram et al. 1994). The [Ne II]12.81 $\mu \mathrm{m}$ velocity field of NGC 3256 appears perturbed due to the merger nature of this galaxy. It is consistent with the $\mathrm{H} \alpha$ velocity field in shape and amplitude. The $\mathrm{H} \alpha$ velocity maps of NGC 7771 and NGC 6701 are slightly smaller than those presented here. NGC 7771 has and ordered velocity field compatible with a rotating disk whereas NGC 6701 presents a complex morphology both in $\mathrm{H} \alpha$ and mid-IR emission lines. The structure and the peak-to-peak velocity amplitude $\left(\sim 300 \mathrm{~km} \mathrm{~s}^{-1}\right.$ for NGC 7771 and $\sim 150 \mathrm{~km} \mathrm{~s}^{-1}$ for NGC 6701) of the $[\mathrm{Ne}$ II $] 12.81 \mu \mathrm{m}$ velocity field are in good agreement with those of the $\mathrm{H} \alpha$ velocity field. As in the previous cases, the $\mathrm{H} \alpha$ velocity field of NGC 7591 is also similar to that of the $[\mathrm{Ne}$ II] $12.81 \mu \mathrm{m}$.

\section{CONCLUSIONS}

We presented the analysis of Spitzer/IRS mapping observations of 15 local LIRGs with extended $\mathrm{Pa} \alpha$ emission. We studied the spatial distribution of the mid-IR spectral features and compared the nuclear and integrated spectra. We calculated fine structure line and PAH ratios which trace the physical conditions in the star-forming regions. The main results are the following.

1. We used the $9.7 \mu \mathrm{m}$ silicate feature strength versus $6.2 \mu \mathrm{m}$ PAH EW diagram to classify the activity of the LIRGs. There is a good agreement with the optical classification and this diagram. Most of the LIRGs populate the pure starburst class and only the nuclei classified as Seyfert from optical spectroscopy appear in the diagram in the AGN/SB class. The integrated values show, in general, larger $6.2 \mu \mathrm{m}$ PAH EW and shallower silicate absorption than the nuclear spectra. That is, the extended star formation partially masks the nuclear activity, resulting in starburst-like integrated spectra. The silicate feature strength, nuclear and integrated, of our sample of local LIRGs $\left(S_{\mathrm{Si}} \sim-0.4\right.$ to -0.9$)$ is small compared to that found in ULIRGs ( $S_{\mathrm{Si}}$ up to -4$)$.

2. We constructed maps of the spatial distribution of the [Ne III] $15.56 \mu \mathrm{m} /[\mathrm{Ne}$ II] $12.81 \mu \mathrm{m}$, which traces the radiation field hardness, and the [S III] $18.71 \mu \mathrm{m} /[\mathrm{Ne}$ II $] 12.81 \mu \mathrm{m}$ that varies with the electron density. In general, the minimum of both ratios is located at the nucleus. The nuclear $[\mathrm{Ne}$ III] $15.56 \mu \mathrm{m} /[\mathrm{Ne}$ II] $12.81 \mu \mathrm{m}$ ratio is below the expected range derived by star formation models. A possible explanation is that due to the high densities in the nuclear regions this ratio is suppressed, including the possibilities that the most massive stars are either missing or buried in ultracompact $\mathrm{H}$ II regions. Alternatively, the star formation rate may have decreased rapidly in all nuclei over the last 10-20 Myr.

3. We find a positive correlation between the [Ne III $] 15.56 \mu \mathrm{m} /$ [Ne II] $12.81 \mu \mathrm{m}$ and [S III] $18.71 \mu \mathrm{m} /[\mathrm{Ne}$ II] $12.81 \mu \mathrm{m}$ ratios for star-forming regions in our sample of LIRGs and other starburst galaxies. On the other hand, AGNs,

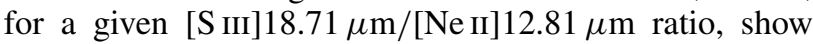
a systematically larger [Ne III] $15.56 \mu \mathrm{m} /[\mathrm{Ne}$ II $] 12.81 \mu \mathrm{m}$ ratio. The few starburst galaxies where we detect the $[\mathrm{S} \mathrm{IV}] 10.51 \mu \mathrm{m}$ emission line follow the correlation between the $[\mathrm{S}$ IV $] 10.51 \mu \mathrm{m} /\left[\mathrm{S}_{\mathrm{III}}\right] 18.71 \mu \mathrm{m}$ and [Ne III] $15.56 \mu \mathrm{m} /[\mathrm{Ne}$ II] $12.81 \mu \mathrm{m}$ observed in $\mathrm{H}$ II regions in other galaxies. We observe larger [S IV] $10.51 \mu \mathrm{m} /$ [S III] $18.71 \mu \mathrm{m}$ ratios for those galaxies harboring an AGN.

4. We find that the $11.3 \mu \mathrm{m}$ PAH emission is more extended than that of the $5.5 \mu \mathrm{m}$ continuum. However, the ratio of the nuclear $(2 \mathrm{kpc}) 6.2 \mu \mathrm{m}$ PAH emission with respect to the integrated emission is comparable to that of the $5.5 \mu \mathrm{m}$ continuum in most cases. We find no correlation between the $[\mathrm{Ne}$ III $] 15.56 \mu \mathrm{m} /[\mathrm{Ne} \mathrm{II}] 12.81 \mu \mathrm{m}$ ratio and the $11.3 \mu \mathrm{m}$ PAH EW, thus the effect of the radiation field hardness in the PAH emission, for the [Ne III] $15.56 \mu \mathrm{m} /$ [Ne II] $12.81 \mu \mathrm{m}$ ratio range in our sample of LIRGs, may not be important. We propose that the $11.3 \mu \mathrm{m} \mathrm{PAH} / 7.7 \mu \mathrm{m}$ PAH ratio depends on the age of the stellar population. 
While the $7.7 \mu \mathrm{m}$ PAH comes from young star-forming regions, the $11.3 \mu \mathrm{m}$ PAH arises also from diffuse medium, thus the $11.3 \mu \mathrm{m}$ PAH/7.7 $\mu \mathrm{m}$ PAH is lower in young H II regions. We also found that large [Ne II] $12.81 \mu \mathrm{m} / 11.3 \mu \mathrm{m}$ $\mathrm{PAH}$ ratios may indicate recent star formation.

5. We explored the variation of the PAH ratios across the galaxies. In most cases these variations are real, that is, they are not due to extinction). In general, the integrated $11.3 \mu \mathrm{m}$ $\mathrm{PAH} / 6.2 \mu \mathrm{m}$ PAH ratios are larger than the nuclear values, probably indicating that the integrated emission includes more neutral conditions. The nuclear and integrated $7.7 \mu \mathrm{m}$ $\mathrm{PAH} / 6.2 \mu \mathrm{m}$ PAH ratios are almost constant, except in the nuclei of galaxies classified as AGN where this ratio is higher. Since the $6.2 \mu \mathrm{m}$ PAH emission is more associated with small molecules, the increased $7.7 \mu \mathrm{m} \mathrm{PAH} / 6.2 \mu \mathrm{m}$ $\mathrm{PAH}$ ratio in AGN could be explained if the small PAH molecules are more easily destroyed in the harsh environments of active nuclei.

6. Using the $\mathrm{H}_{2} \mathrm{~S}(0)$ at $28.2 \mu \mathrm{m}$ and the $\mathrm{H}_{2} \mathrm{~S}(1)$ at $17.0 \mu \mathrm{m}$ lines integrated over $13^{\prime \prime} \times 13^{\prime \prime}(3-5 \mathrm{kpc})$ we estimated the mass of the warm $(T \sim 300 \mathrm{~K})$ molecular hydrogen. It ranges from $(0.4-2.6) \times 10^{8} M_{\odot}$, and they are similar to those of ULIRGs, local starbursts, and Seyfert galaxies. However, these masses are lower limits since it is only included the $\mathrm{H}_{2}$ emission from the central few kpc of the galaxies and the total mass of warm molecular hydrogen is likely to be, at least, a factor of 2 larger.

7. The similarity between the PAHs and molecular hydrogen morphologies suggests that the main excitation mechanism of the latter is the UV radiation too. However there are some regions with an excess of $\mathrm{H}_{2}$ emission with respect to the PAH emission, thus the other mechanisms should contribute noticeably to the $\mathrm{H}_{2}$ emission. Some of these regions are associated with interacting systems, where large-scale shocks may also play a role in exciting the molecular hydrogen.

8. Despite the modest spectral resolution of the SH module, we show that useful velocity information can be obtained from the SH spectra. For most of the galaxies and on the physical scales probed by the IRS spectra $(\sim \mathrm{kpc})$ the velocity fields are comparable with that produced by a rotating disk. For the galaxies with available $\mathrm{H} \alpha$ velocity fields, the $[\mathrm{Ne}$ II] $12.81 \mu \mathrm{m}$ velocity fields are in good agreement with those of the $\mathrm{H} \alpha$, both in shape and peak-to-peak velocities.

The authors thank Takashi Hattori, M. Agúndez, B. Groves, and Dimitra Rigopoulou for their help and enlightening discussions, as well as to Miwa Block for producing some of the data cubes. We thank the anonymous referee for the useful comments and suggestions, which significantly improved this paper.

M.P.-S. acknowledges support from the CSIC under grant JAE-Predoc-2007. Support for this work was provided by NASA through contract 1255094 issued by JPL/California Institute of Technology. M.P.-S., A.A.-H., L.C., and T.D.-S. acknowledge support from the Spanish Plan Nacional del Espacio under grant ESP2007-65475-C02-01. A.A.-H. also acknowledges support for this work from the Spanish Ministry of Science and Innovation through Proyecto Intramural Especial under grant number 200850I003. T.D.S. acknowledges support from the Consejo Superior de Investigaciones Científicas under grant I3P-BPD-2004. P.G.P.-G. acknowledges support from the
Table 9

Log of IRS Mapping Calibration Observations

\begin{tabular}{lcccc}
\hline \hline Module & AOR & Target & $\begin{array}{c}\text { Step Size Perpendicular } \\
\text { (arcsec) }\end{array}$ & $\begin{array}{c}\text { Step Size Parallel } \\
\text { (arcsec) }\end{array}$ \\
\hline SL1, SL2 & 16295168 & HR 7341 & 0.6 & 15.5 \\
LL1, LL2 & 16463104 & HR 6606 & 1.7 & 46.0 \\
SH & 16294912 & HR 6688 & 0.8 & 0.8 \\
SH & 16340224 & sky & & 1.7 \\
LH & 16101888 & HR 2491 & 1.7 & \\
LH & 116088320 & sky & & \\
\hline
\end{tabular}

Ramón y Cajal Program financed by the Spanish Government and the European Union, and from the Spanish Programa Nacional de Astronomía y Astrofísica under grants AYA 200602358 and AYA 2006-15698-C02-02. This research has made use of the NASA/IPAC Extragalactic Database (NED), which is operated by the Jet Propulsion Laboratory, California Institute of Technology, under contract with the National Aeronautics and Space Administration.

\section{APPENDIX A}

\section{THE POINT SPREAD FUNCTION OF IRS SPECTRAL MAPPING OBSERVATIONS}

When comparing maps of lines or features, at different wavelengths, the variation of the PSF size can be significant. The Spitzer/IRS PSF is much less studied than that of the other Spitzer instruments, although it is possible to generate synthetic IRS PSF using STinyTim program (Krist 2002). This is useful for staring observations, but the spectral mapping observation mode introduces extra issues, such as the cube assembly from staring observations, wavelength calibration uncertainties, that cannot be easily taken into account using the modeled PSF. Because of that, a better way to characterize the IRS PSF of the spectral mapping observation mode is to use the mapping observations of calibration stars available in the Spitzer archive. Previously, Kóspál et al. (2008) studied the factor that should be applied to correct for flux losses when the slit is not centered properly on a point source. We extend this analysis to characterize the variations of the PSF.

To do this, we used the same data sets as Kóspál et al. (2008), of spectral mapping observations of calibration stars. These observations were taken with the same instrument configuration used for the galaxies studied in this paper, but with much smaller steps between pointings, i.e., the calibration observations have a better spatial information of the PSF. Details of the observations are listed in Table 9.

After obtaining BCD files processed by Spitzer pipeline (version 15.3 for SH and SL modules, and version 17.2 for LH and LL modules), we built the spectral cubes with CUBISM (Smith et al. 2007a). The sky emission was subtracted using dedicated background observations. To take advantage of the better spatial information of the calibration observations, we set the pixel size in the final cube to be the size of the step in the perpendicular direction ${ }^{10}$ (see Table 9). We did this for all IRS modules, however we only present the results for the SH, SL1, and SL2 modules, because the other modules (LH, LL1, and LL2), are heavily affected by fringing patterns and their PSF is not well reproduced by a Gaussian.

\footnotetext{
10 In this section we use the terms: "perpendicular" and "parallel" directions. They refer to the direction along the spectral dispersion axis and the spatial axis of the slit, respectively.
} 

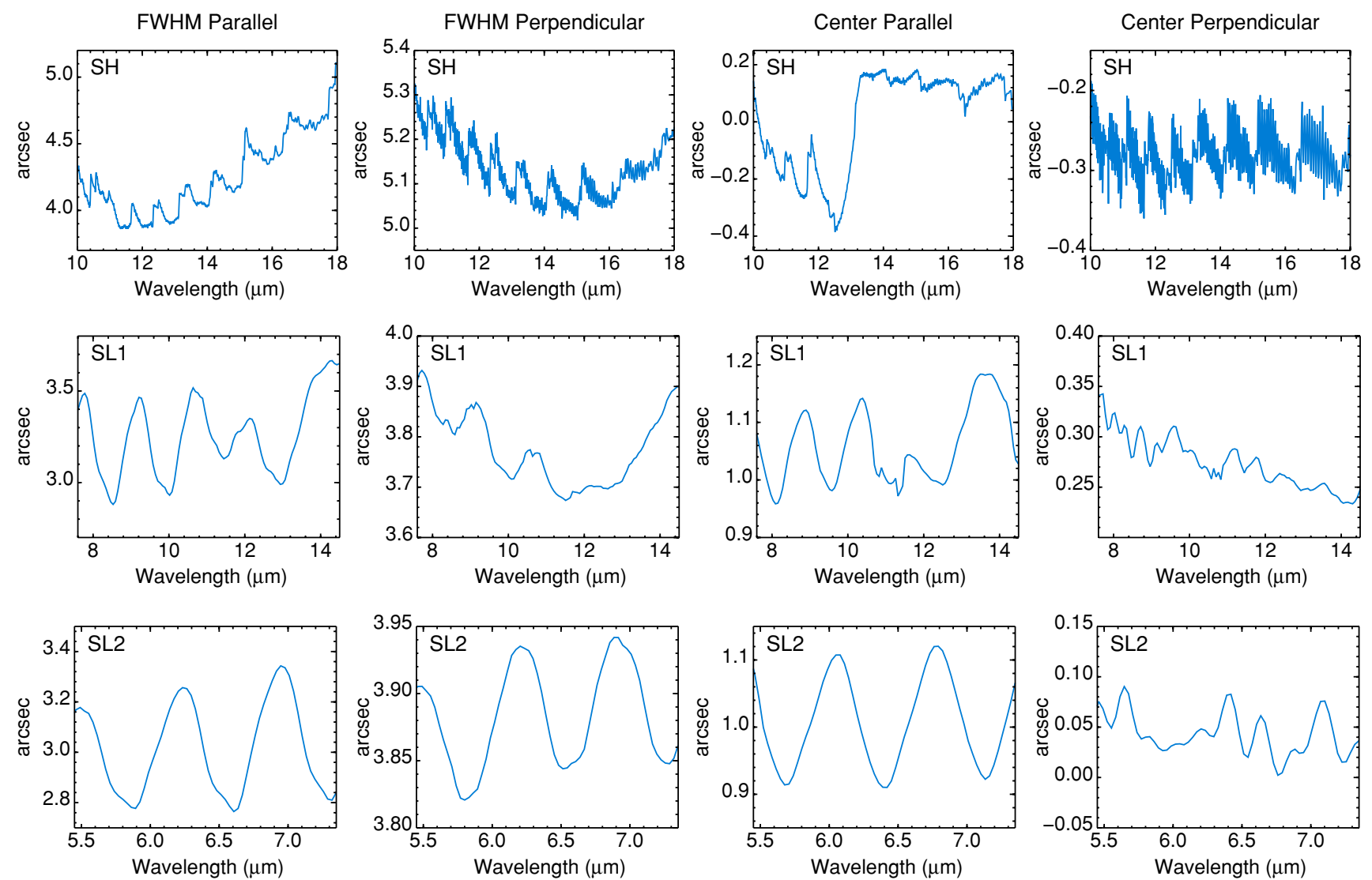

Figure 25. Full width at half-maximum (FWHM) and centroid position of the PSF of the modules SH, SL1, and SL2. Parallel and perpendicular directions are represented. We have calculated these values by fitting a two-dimensional Gaussian.

(A color version of this figure is available in the online journal.)

In order to study the PSF core variations we fitted each plane of the cube using a two-dimensional Gaussian. We allowed different values of the full width at half-maximum (FWHM) for each axis of the Gaussian, but the axis orientation is fixed and aligned with the parallel and perpendicular directions. By doing this we obtained the FWHM and centroid of the PSF at every wavelength. These values are represented in Figure 25.

The undulating pattern of the FWHM and the centroid in the modules SL1 and SL2 is originated by several causes. (1) The spectral axis is not aligned with the detector; thus to extract the spectra is necessary to resample the original image. This resampling process produces this kind of periodic patterns. (2) The algorithm used to build the data cubes (see Smith et al. 2007a for details) does not try to correct for this effect. In any case, these variations are small: $<10 \%$ for the FWHM and $<0$ '. 2 for the centroid position.

The PSF core is slightly asymmetric for all modules. The FWHM in the perpendicular direction is larger than the FWHM in the parallel direction, which has an almost constant value. A possible explanation to this is the way CUBISM builds the cubes. It assumes that there is no spatial information in the dispersion (or perpendicular) direction and the spatial information in that direction comes from different telescope pointings (for more details see Smith et al. 2007a). The averaged values of the FWHM of each module are listed in Table 10.

The variation of the centroid position in SL modules is small. The $1 \sigma$ deviation around the average centroid position are 0.07 and $0{ }^{\prime} .02$ for the parallel and perpendicular directions respectively, that is a 20th of the SL pixel size. These variations
Table 10

IRS PSF Full Width Half Maximum

\begin{tabular}{lcc}
\hline \hline Module & $\begin{array}{c}\text { FWHM Perpendicular } \\
(\operatorname{arcsec})\end{array}$ & $\begin{array}{c}\text { FWHM Parallel } \\
(\operatorname{arcsec})\end{array}$ \\
\hline SH & $5.16 \pm 0.08$ & $4.31 \pm 0.37$ \\
SL1 & $3.76 \pm 0.07$ & $3.27 \pm 0.21$ \\
SL2 & $3.88 \pm 0.04$ & $3.02 \pm 0.17$ \\
\hline
\end{tabular}

can be attributed to small telescope pointing errors and signal-tonoise limitation. The position of the centroid in the SH module shows variations that cannot be explained just by instrumental pointing error or a limited signal to noise. The $1 \sigma$ deviation in the perpendicular direction is 0.03 , but in the parallel direction the variation is one order of magnitude larger $\left(0^{\prime \prime} 2\right)$ and shows a clear wavelength dependency. The most probable cause for this, is an error in the position and/or angle of the extraction aperture. However this effect (0".6) is small when compared with the SH pixel size (2".26).

We studied the variations of the diffraction rings in the SH module. We fitted the central row of the cube for each wavelength using a Gaussian for the PSF core and two Gaussians for the only diffraction ring detected. These two Gaussians have the same FWHM and they are symmetrically placed around the PSF core position. Figure 26 shows a fit example. The results of the fits are summarized in the Figure 27. The FWHM of the diffraction ring, that does not depend on the wavelength, is 3".6 \pm 0 ' 3 . Instead, the position of the ring depends linearly on wavelength. The relative value of the diffraction ring peak with 

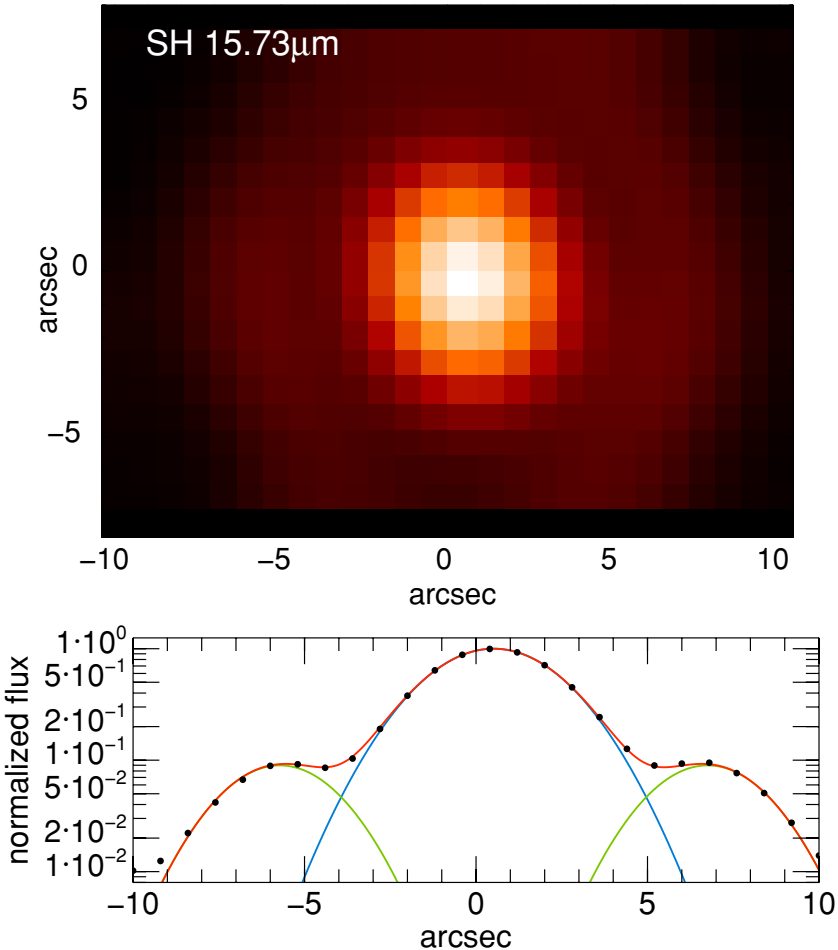

Figure 26. Upper panel: PSF of the SH module at $15.73 \mu \mathrm{m}$. Lower panel: fit of the PSF profile at the central row. The blue line is the Gaussian used to fit the PSF core. The green line is the fit of the diffraction ring. The red line is the sum of both components.

(A color version of this figure is available in the online journal.)

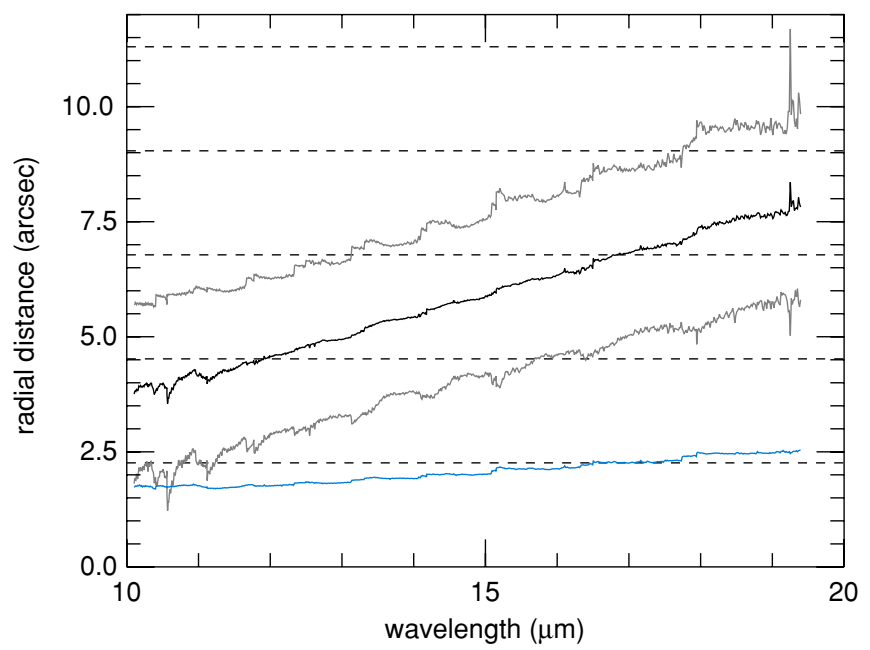

Figure 27. Position of the diffraction ring in the SH module (black solid line). The position of the half-maximum of the diffraction ring is shown in gray. For comparison, the blue line is the half width at half maximum of the PSF core. The dashed black lines represent the pixel size of the SH module.

(A color version of this figure is available in the online journal.)

respect to the PSF core peak depends slightly on wavelength. At $10 \mu \mathrm{m}$ it is $11 \%$ of the core peak value and at $19 \mu \mathrm{m}$ it is $9 \%$.

When calculating feature ratios, the PSF variations (FWHM and centroid), could originate artifacts. For the SL module these variations are less than one tenth of the pixel size and should not affect feature ratios. For the SH module the major source of uncertainty is the centroid position, but the difference is only one fourth of the pixel size. Since these variations are very small compared with the pixel size, they do not affect the calculated feature ratios. Diffraction rings can produce artifacts in the feature ratios too. They cause inaccurate ratio values 3 or 4 pixels away from the center of the point source in the $\mathrm{SH}$ module. For extended sources the situation is more complex because it is not possible to know where the diffraction ring will appear. However, comparing the spatial profiles of the features and the underlying continuum, as we do in this paper, helps determine if the ratio values are real or artifacts.

\section{APPENDIX B}

\section{DESCRIPTION OF INDIVIDUAL OBJECTS}

\section{B.1. NGC 2369}

The MIR emission of NGC 2369 is dominated by the resolved nucleus. The molecular hydrogen $\left(\mathrm{H}_{2} \mathrm{~S}(1)\right.$ and $\mathrm{H}_{2}$ $\mathrm{S}(2)$ ) emission appears more extended along the galaxy disk than the $11.3 \mu \mathrm{m}$ PAH emission. This causes a relatively large $\mathrm{H}_{2} \mathrm{~S}(1) / 11.3 \mu \mathrm{m}$ PAH ratio in the regions around the nucleus. The $\left[\mathrm{Ne}\right.$ II] $12.81 \mu \mathrm{m} / 11.3 \mu \mathrm{m}$ PAH ratio map reveals an $\mathrm{H}_{\mathrm{II}}$ region $\sim 17$ arcsec $(\sim 3.4 \mathrm{kpc}$ projected $)$ away from the nucleus to the south. The nuclear silicate strength, $S_{\mathrm{Si}} \sim 0.9$, is slightly larger than the average silicate strength of our LIRGs sample, probably because this galaxy is seen almost edge-on.

\section{B.2. NGC 3110}

It is a face-on galaxy with numerous $\mathrm{H}$ II regions along the spiral arms (Hattori et al. 2004). The [Ne III] $15.56 \mu$ m emission of this galaxy is especially bright and it resembles that of the $\mathrm{H} \alpha$ emission. The [Ne III $] 15.56 \mu \mathrm{m} /[\mathrm{Ne} \mathrm{II}] 12.81 \mu \mathrm{m}$ ratio of the extranuclear $\mathrm{H}$ II regions is $\sim 5$ times larger than the nuclear ratio. The deepest silicate absorption is located at the nucleus.

\section{B.3. NGC 3256}

It is galaxy merger with two nuclei. The southern nucleus, located 5 arcsec away from the north nucleus, is highly obscured, although it is visible in the $5.5 \mu \mathrm{m}$ continuum map. It has one of the deepest silicate absorption feature in our sample, $S_{\mathrm{Si}} \sim-1.4$. The silicate feature of the rest of the galaxy is similar to that found in the rest of the galaxies of our sample. The $\mathrm{PAH}$, continuum and atomic emission (except [S IV] $10.51 \mu \mathrm{m}$ ) peak at the north nucleus. The maximum of the [S IV] $10.51 \mu \mathrm{m}$ emission is located $\sim 5$ arcsec to the east of the north nucleus, and it is probably related to a star-forming region. This is the only extranuclear region $(d>\sim 1 \mathrm{kpc})$, apart from region $\mathrm{C}$ in Arp 299 (Alonso-Herrero et al. 2009b), where we detect the [S IV] $10.51 \mu \mathrm{m}$ line. The $\mathrm{H}_{2}$ emission arises mainly from the southern nucleus and the surrounding regions.

\section{B.4. ESO 320-G030}

Due to the low spatial resolution of the IRS maps, we cannot distinguish any morphological detail in the emission maps this galaxy, although it is clearly spatially resolved. The maximum of the silicate feature absorption is located at the nucleus.

\section{B.5. NGC 5135}

This galaxy hosts a Sy2 nucleus surrounded by intense star formation (Bedregal et al. 2009 and references therein) that we cannot separate due to the limited spatial resolution of the IRS spectral maps. However the line ratios (e.g., [Ne III]15.56 $\mu \mathrm{m} /$ 
$[\mathrm{Ne}$ II $] 12.81 \mu \mathrm{m}$ ratio) show some asymmetries that may be associated with the truncated ring-like structure of this galaxy. At our scale $(\sim 0.5 \mathrm{kpc})$, we do not see any noticeable variations in the PAH emission due to the AGN activity. The silicate absorption feature is shallow $(-0.47)$ and the maximum value is not located at the nucleus.

\section{B.6. IC $4518 E$}

This galaxy is part of an interacting pair (IC 4518E/IC $4518 \mathrm{~W}$ ). Most of the mid-infrared emission (PAH, fine structure line and $15 \mu \mathrm{m}$ continuum) emission is dominated by an $\mathrm{H}_{\text {II }}$ region located $\sim 7$ arcsec to the southeast from the nucleus. The nuclear bright is similar to that of the southwestern $\mathrm{H}$ II region emission only for the molecular hydrogen $\left(\mathrm{H}_{2} \mathrm{~S}(1)\right)$ and the $5.5 \mu \mathrm{m}$ continuum. The deepest silicate absorption is found at the nucleus.

\section{B.7. $Z w 049.057$}

Due to the low $\mathrm{S} / \mathrm{N}$ of the observations of $\mathrm{Zw} 049.057$, the maps of this galaxy contain very few pixels. The nuclear silicate absorption is, $S_{\mathrm{Si}}=-1.2$, which is large when compared to that of the other LIRGs.

\section{B.8. IRAS $17138-1017$}

The limited spatial resolution of the emission maps does not allow us to identify any morphological details in this galaxy. The maximum of the silicate absorption may not be located at the nucleus. Note however that the near-infrared (AlonsoHerrero et al. 2006) and the mid-infrared (Díaz-Santos et al. 2008) morphologies do not allow us to determine the position of the nucleus.

\section{B.9. IC 4687}

IC 4687 is part of an interacting system (IC 4686/IC 4687). Only IC 4687 was observed in the spectral mapping mode. In the emission line map it is not possible to isolate any region of interest, however the emission is spatially resolved. We detect the [S IV] $10.51 \mu \mathrm{m}$ line, that seems to be associated with the nuclear H II regions seen in the Pa $\alpha$ image. Two of these regions have slightly larger [Ne II] $12.81 \mu \mathrm{m} / 11.3 \mu \mathrm{m}$ PAH ratio than the rest of the galaxy. The nuclear [Ne III] $15.56 \mu \mathrm{m} /$ [Ne II] $12.81 \mu \mathrm{m}$ ratio of this galaxy is somewhat larger than those of the other $\mathrm{H}$ II galaxies in our sample. The maximum of the silicate feature is not located at the nucleus.

\section{B.10. NGC 6701}

This LIRG is classified as composite (H II/LINER) from optical spectroscopy (Alonso-Herrero et al. 2009a). We can identify two extranuclear $\mathrm{H}$ II regions symmetrically located $\sim 10$ arcsec away from the nucleus, one at each spiral arm. As is the case with the other extranuclear H II regions, the molecular hydrogen of these two regions is relatively low when compared with the $\mathrm{H}_{2}$ nuclear emission. The silicate absorption is deeper in the nucleus than in the rest of the galaxy.

\section{B.11. NGC 7130}

This is a face-on galaxy whose nuclear activity is classified as Sy/LINER. We detect two extranuclear $\mathrm{H}$ in regions located in the spiral arms. The brightest is located to the north, $\sim 10 \operatorname{arcsec}$ from the nucleus. The other one is $\sim 14 \operatorname{arcsec}$ to the southeast from the nucleus. The continuum and [Ne III] $15.56 \mu \mathrm{m}$ emissions are concentrated at the nucleus. The PAH features and fine structure lines are bright in the spiral arms too. The minimum of the $[\mathrm{Ne}$ III] $15.56 \mu \mathrm{m} /[\mathrm{Ne}$ II] $12.81 \mu \mathrm{m}$ ratio is not located at the nucleus, as for the majority of the LIRGs in our sample. We detect nuclear [S IV] $10.51 \mu \mathrm{m}$ emission. Also, this is one of the few galaxies in the sample whose $11.3 \mu \mathrm{m} \mathrm{PAH} / 6.2 \mu \mathrm{m}$ PAH ratio minimum is not located at the nucleus. These facts may be related to the active nature of the nucleus of this galaxy.

$$
\text { B.12. IC } 5179
$$

This is a face-on spiral galaxy classified as H II-like from optical spectroscopy, and it shows numerous $\mathrm{H}$ II regions distributed in the spiral arms (Alonso-Herrero et al. 2006). These $\mathrm{H}$ II regions are not resolved in the Spitzer maps. They appear as a relatively uniform extended emission (PAH features and atomic lines) over $\sim 8 \mathrm{kpc}$. The continuum emission is slightly more concentrated toward the nucleus. The patterns in the ratio maps are possibly related to the intricate distribution of the $\mathrm{H}_{\text {II }}$ regions. The nucleus is the most obscured region of this galaxy.

\section{B.13. NGC 7591}

The nuclear activity of NGC 7591 is classified as composite (Alonso-Herrero et al. 2009a). Only in the low spectral resolution maps we can clearly distinguish the spiral arms of this face-on galaxy. The continuum emission is concentrated at the nucleus, while we can see bright PAH and fine structure lines emission in the galaxy arms. The nucleus is the region with the deepest silicate absorption feature.

\section{B.14. NGC 7771}

The nucleus of this LIRG is classified as H II-like from optical spectroscopy. It has a nuclear ring of star formation clearly seen in the $\mathrm{Pa} \alpha$ images (Figure 4; see also Alonso-Herrero et al. 2006, 2009a), but it is not resolved by the IRS observations. This galaxy is seen almost edge-on and we can clearly observe the spiral arms in the line emission maps. We detect an H II region in the eastern arm, $\sim 20 \operatorname{arcsec}(6 \mathrm{kpc})$ away from the nucleus. The continuum is dominated by the nucleus. The $\mathrm{H}_{2}$ emission extends $\sim 5 \mathrm{kpc}$ in the west galaxy arm with apparently no bright fine structure/PAH emission associated with it. The silicate feature spatial distribution is somewhat irregular and we do not see any morphological detail.

\section{REFERENCES}

Aalto, S., Radford, S. J. E., Scoville, N. Z., \& Sargent, A. I. 1997, ApJ, 475, L107

Alonso-Herrero, A., García-Marín, M., Monreal-Ibero, A., Colina, L., Arribas, S., Alfonso-Garzón, J., \& Labiano, A. 2009a, A\&A, 506, 1541

Alonso-Herrero, A., Pereira-Santaella, M., Rieke, G. H., Colina, L., Engelbracht, C. W., Pérez-González, P. G., Díaz-Santos, T., \& Smith, J. 2010, Adv. Space Res., 45, 99

Alonso-Herrero, A., Rieke, G. H., Rieke, M. J., Colina, L., Pérez-González, P. G., \& Ryder, S. D. 2006, ApJ, 650, 835

Alonso-Herrero, A., Rieke, G. H., Rieke, M. J., \& Scoville, N. Z. 2000, ApJ, 532,845

Alonso-Herrero, A., et al. 2009b, ApJ, 697, 660

Amram, P., Marcelin, M., Balkowski, C., Cayatte, V., Sullivan, W. T., III, \& Le Coarer, E. 1994, A\&AS, 103, 5

Armus, L., et al. 2007, ApJ, 656, 148

Armus, L., et al. 2009, PASP, 121, 559

Augarde, R., \& Lequeux, J. 1985, A\&A, 147, 273

Bedregal, A. G., Colina, L., Alonso-Herrero, A., \& Arribas, S. 2009, ApJ, 698, 1852

Beirão, P., et al. 2008, ApJ, 676, 304 
Brandl, B. R., et al. 2006, ApJ, 653, 1129

Caputi, K. I., et al. 2007, ApJ, 660, 97

Casoli, F., Willaime, M., Viallefond, F., \& Gerin, M. 1999, A\&A, 346, 663

Dale, D. A., et al. 2006, ApJ, 646, 161

Dale, D. A., et al. 2009, ApJ, 693, 1821

Devost, D., et al. 2004, ApJS, 154, 242

Díaz-Santos, T., Alonso-Herrero, A., Colina, L., Packham, C., Levenson, N. A., Pereira-Santaella, M., Roche, P. F., \& Telesco, C. M. 2010, ApJ, 711, 328

Díaz-Santos, T., Alonso-Herrero, A., Colina, L., Packham, C., Radomski, J. T., \& Telesco, C. M. 2008, ApJ, 685, 211

Dopita, M. A., et al. 2006, ApJS, 167, 177

Draine, B. T., \& Li, A. 2001, ApJ, 551, 807

Engelbracht, C. W., Rieke, G. H., Gordon, K. D., Smith, J.-D. T., Werner, M. W., Moustakas, J., Willmer, C. N. A., \& Vanzi, L. 2008, ApJ, 678, 804

Farrah, D., et al. 2007, ApJ, 667, 149

Farrah, D., et al. 2008, ApJ, 677, 957

Galliano, F. 2006, arXiv:astro-ph/0610852

Galliano, F., Madden, S. C., Tielens, A. G. G. M., Peeters, E., \& Jones, A. P. 2008, ApJ, 679, 310

García-Marín, M., Colina, L., Arribas, S., Alonso-Herrero, A., \& Mediavilla, E. 2006, ApJ, 650, 850

Genzel, R., et al. 1998, ApJ, 498, 579

Giveon, U., Sternberg, A., Lutz, D., Feuchtgruber, H., \& Pauldrach, A. W. A. 2002, ApJ, 566, 880

Gordon, K. D., Engelbracht, C. W., Rieke, G. H., Misselt, K. A., Smith, J.-D. T., \& Kennicutt, R. C., Jr. 2008, ApJ, 682, 336

Groves, B. A., Dopita, M. A., \& Sutherland, R. S. 2004, ApJS, 153, 75

Habart, E., Boulanger, F., Verstraete, L., Pineau des Forêts, G., Falgarone, E., \& Abergel, A. 2003, A\&A, 397, 623

Hattori, T., et al. 2004, AJ, 127, 736

Higdon, S. J. U., Armus, L., Higdon, J. L., Soifer, B. T., \& Spoon, H. W. W. 2006, ApJ, 648, 323

Ho, L. C., \& Keto, E. 2007, ApJ, 658, 314

Hollenbach, D., \& McKee, C. F. 1989, ApJ, 342, 306

Houck, J. R., et al. 2004, ApJS, 154, 18

Imanishi, M., Dudley, C. C., Maiolino, R., Maloney, P. R., Nakagawa, T., \& Risaliti, G. 2007, ApJS, 171, 72

Kóspál, Á., Ábrahám, P., Apai, D., Ardila, D. R., Grady, C. A., Henning, T., Juhász, A., Miller, D. W., \& Moór, A. 2008, MNRAS, 383, 1015

Krist, J. 2002, Tiny Tim/SIRTF User's Guide (Pasadena, CA: SSC)

Le Floc'h, E., et al. 2005, ApJ, 632, 169

Lepp, S., \& McCray, R. 1983, ApJ, 269, 560

Lípari, S., Díaz, R., Taniguchi, Y., Terlevich, R., Dottori, H., \& Carranza, G. 2000, AJ, 120, 645
Markwardt, C. B. 2009, in ASP Conf. Ser. 411, Astronomical Data Analysis Software and Systems XVIII, ed. D. A. Bohlender, D. Durand, \& P. Dowler (San Francisco, CA: ASP), 251

Menéndez-Delmestre, K., et al. 2009, ApJ, 699, 667

Nardini, E., Risaliti, G., Salvati, M., Sani, E., Imanishi, M., Marconi, A., \& Maiolino, R. 2008, MNRAS, 385, L130

Peeters, E., Spoon, H. W. W., \& Tielens, A. G. G. M. 2004, ApJ, 613, 986

Pérez-González, P. G., et al. 2005, ApJ, 630, 82

Pope, A., Chary, R.-R., Dickinson, M., \& Scott, D. 2007, in ASP Conf. Ser. 380, Deepest Astronomical Surveys, ed. J. Afonso, H. C. Ferguson, B. Mobasher, \& R. Norris (San Francisco, CA: ASP), 387

Rieke, G. H., \& Lebofsky, M. J. 1985, ApJ, 288, 618

Rieke, G. H., \& Low, F. J. 1972, ApJ, 176, L95

Rigby, J. R., et al. 2008, ApJ, 675, 262

Rigby, J. R., \& Rieke, G. H. 2004, ApJ, 606, 237

Rigopoulou, D., Kunze, D., Lutz, D., Genzel, R., \& Moorwood, A. F. M. 2002, A\&A, 389, 374

Roussel, H., et al. 2007, ApJ, 669, 959

Rupke, D. S. N., Veilleux, S., \& Baker, A. J. 2008, ApJ, 674, 172

Sanders, D. B., Mazzarella, J. M., Kim, D.-C., Surace, J. A., \& Soifer, B. T. 2003, AJ, 126, 1607

Sanders, D. B., \& Mirabel, I. F. 1996, ARA\&A, 34, 749

Shaw, G., Ferland, G. J., Abel, N. P., Stancil, P. C., \& van Hoof, P. A. M. 2005, ApJ, 624, 794

Sirocky, M. M., Levenson, N. A., Elitzur, M., Spoon, H. W. W., \& Armus, L. 2008, ApJ, 678, 729

Smith, J. D. T., et al. 2007a, PASP, 119, 1133

Smith, J. D. T., et al. 2007b, ApJ, 656, 770

Snijders, L., Kewley, L. J., \& van der Werf, P. P. 2007, ApJ, 669, 269

Soifer, B. T., et al. 2000, AJ, 119, 509

Spoon, H. W. W., Marshall, J. A., Houck, J. R., Elitzur, M., Hao, L., Armus, L., Brandl, B. R., \& Charmandaris, V. 2007, ApJ, 654, L49

Surace, J. A., Sanders, D. B., \& Mazzarella, J. M. 2004, AJ, 127, 3235

Thornley, M. D., Schreiber, N. M. F., Lutz, D., Genzel, R., Spoon, H. W. W., Kunze, D., \& Sternberg, A. 2000, ApJ, 539, 641

Tommasin, S., Spinoglio, L., Malkan, M. A., Smith, H., González-Alfonso, E., \& Charmandaris, V. 2008, ApJ, 676, 836

Veilleux, S., Kim, D.-C., Sanders, D. B., Mazzarella, J. M., \& Soifer, B. T. 1995, ApJS, 98, 171

Veilleux, S., et al. 2009, ApJS, 182, 628

Velusamy, T., \& Langer, W. D. 2008, AJ, 136, 602

Verma, A., Lutz, D., Sturm, E., Sternberg, A., Genzel, R., \& Vacca, W. 2003, A\&A, 403, 829

Wu, Y., Charmandaris, V., Hao, L., Brandl, B. R., Bernard-Salas, J., Spoon, H. W. W., \& Houck, J. R. 2006, ApJ, 639, 157 